Florida International University FIU Digital Commons

$10-30-2007$

\title{
Connecting visual and analytic reasoning to improve students' spatial visualization abilities : a constructivist approach
}

Mario Eraso

Florida International University

DOI: $10.25148 /$ etd.FI15101303

Follow this and additional works at: https://digitalcommons.fiu.edu/etd

Part of the Curriculum and Instruction Commons

\section{Recommended Citation}

Eraso, Mario, "Connecting visual and analytic reasoning to improve students' spatial visualization abilities : a constructivist approach" (2007). FIU Electronic Theses and Dissertations. 3146.

https://digitalcommons.fiu.edu/etd/3146 


\section{CONNECTING VISUAL AND ANALYTIC REASONING TO IMPROVE STUDENTS' SPATIAL VISUALIZATION ABILITIES: A CONSTRUCTIVIST APPROACH}

A dissertation submitted in partial fulfillment of the requirements for the degree of DOCTOR OF PHILOSOPHY in CURRICULUM AND INSTRUCTION by Mario Eraso 2007 
To: Dean Luis Mirón

College of Education

This dissertation, written by Mario Eraso, and entitled Connecting Visual and Analytic Reasoning to Improve Students' Spatial Visualization Abilities: A Constructivist Approach, having been approved in respect to style and intellectual content, is referred to you for judgment.

We have read this dissertation and recommend that it be approved.

Kingsley Banya

Sneh Gulati

Lisa Delpit, Co-Major Professor

George E. O'Brien, Co-Major Professor

Date of Defense: October 30, 2007

The dissertation of Mario Eraso is approved.

Dean Luis Mirón

College of Education

Dean George Walker University Graduate School

Florida International University, 2007 
(C) Copyright 2007 by Mario Eraso

All rights reserved. 


\section{DEDICATION}

I dedicate this dissertation to my friend Omar. 


\section{ACKNOWLEDGMENTS}

I thank the members of my committee for their support, patience, and guidance. Dr. Sneh Gulati was very helpful in assisting me with the analysis and interpretations necessary in quantitative methodology. Dr. Kingsley Banya's support and advice was important throughout the last months of this process. Dr. Lisa Delpit's invaluable assistance in polishing the manuscript is also greatly appreciated. Finally, I thank Dr. George E. O'Brien for walking me through an arduous process. The numerous hours he spent with me to bring this study to completion with excellence will always be appreciated.

I also wish to thank Joannie Wynne, for her emotional support. She was a true cheerleader. To Bob Moses, from the Algebra Project, and to Greg Bowe and Alex Salinas, from the Center for Urban Education and Innovation, many thanks for listening to me. Professors Edwin McClintock and Zhonghong Jiang were also instrumental in providing the foundations for the study. I am thankful to their program Partnership for Academic Communities (PAC) at the College of Education. To PAC teachers Martha Delgado, Yanet Garcia, and Giselle Jorge, thank you very much for your assistance and friendship.

Finally, I want to thank Anabel, my wife, for everything. 


\section{ABSTRACT OF THE DISSERTATION \\ CONNECTING VISUAL AND ANALYTIC REASONING \\ TO IMPROVE STUDENTS' SPATIAL VISUALIZATION ABILITIES:}

\section{A CONSTRUCTIVIST APPROACH}

by

\section{Mario Eraso}

Florida International University, 2007

Miami, Florida

Professor George E. O'Brien, Co-Major Professor

Professor Lisa Delpit, Co-Major Professor

Current reform initiatives recommend that school geometry teaching and learning include the study of three-dimensional geometric objects and provide students with opportunities to use spatial abilities in mathematical tasks. Two ways of using Geometer's Sketchpad (GSP), a dynamic and interactive computer program, in conjunction with manipulatives enable students to investigate and explore geometric concepts, especially when used in a constructivist setting. Research on spatial abilities has focused on visual reasoning to improve visualization skills. This dissertation investigated the hypothesis that connecting visual and analytic reasoning may better improve students' spatial visualization abilities as compared to instruction that makes little or no use of the connection of the two.

Data were collected using the Purdue Spatial Visualization Tests (PSVT) administered as a pretest and posttest to a control and two experimental groups. Sixtyfour $10^{\text {th }}$ grade students in three geometry classrooms participated in the study during 6 
weeks. Research questions were answered using statistical procedures. An analysis of covariance was used for a quantitative analysis, whereas a description of students' visualanalytic processing strategies was presented using qualitative methods. The quantitative results indicated that there were significant differences in gender, but not in the group factor. However, when analyzing a sub sample of 33 participants with pretest scores below the $50^{\text {th }}$ percentile, males in one of the experimental groups significantly benefited from the treatment. A review of previous research also indicated that students with low visualization skills benefited more than those with higher visualization skills. The qualitative results showed that girls were more sophisticated in their visual-analytic processing strategies to solve three-dimensional tasks.

It is recommended that the teaching and learning of spatial visualization start in the middle school, prior to students' more rigorous mathematics exposure in high school. A duration longer than 6 weeks for treatments in similar future research studies is also recommended. 


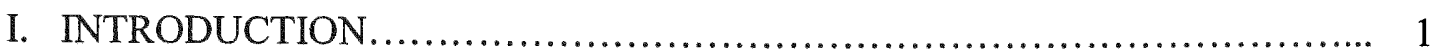

Background of the Problem ..............................................................................

Statement of the Problem ......................................................................................... 9

Research Questions ...................................................................................... 9

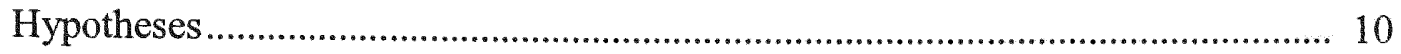

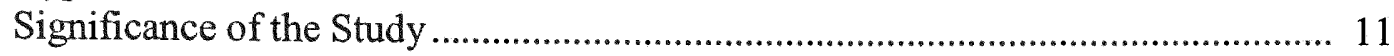

Assumptions of the Study ............................................................................... 12

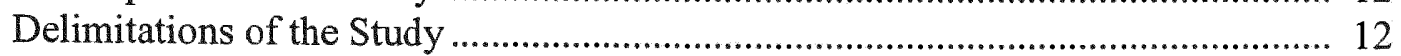

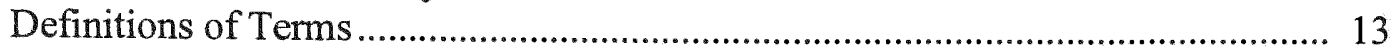

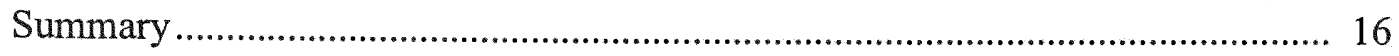

II. REVIEW OF THE LITERATURE ............................................................ 18

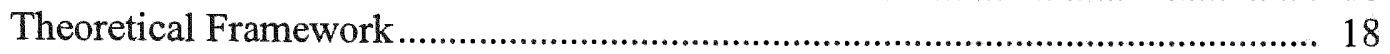

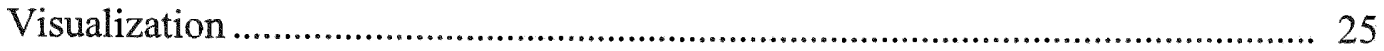

Spatial Abilities and Strategies ....................................................................... 33

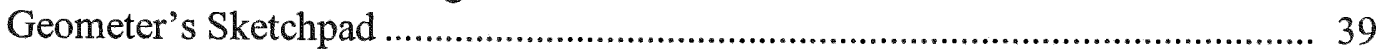

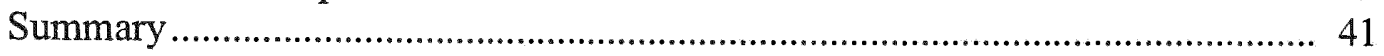

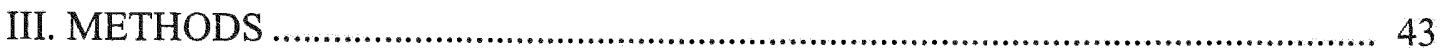

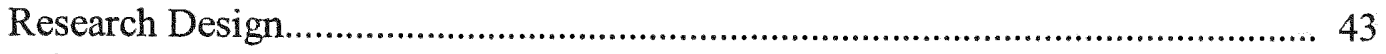

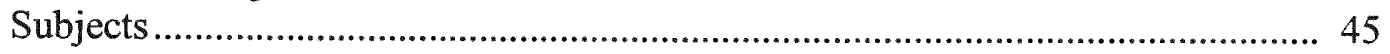

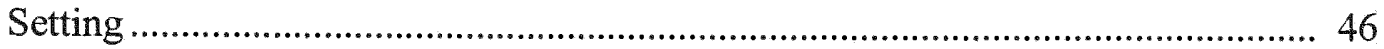

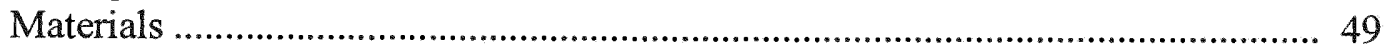

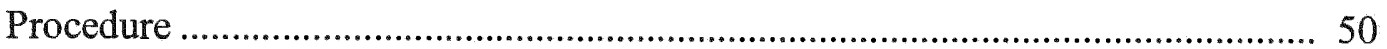

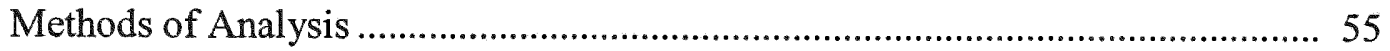

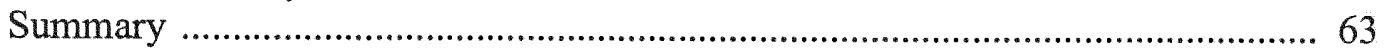

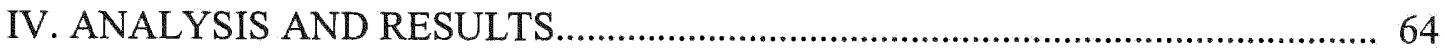

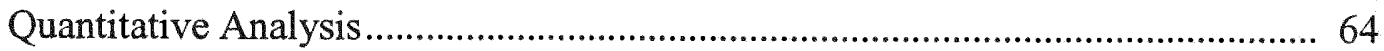

Qualitative Analysis....................................................................................... 71

V. DISCUSSION, CONCLUSION, AND RECOMMENDATIONS .........................100

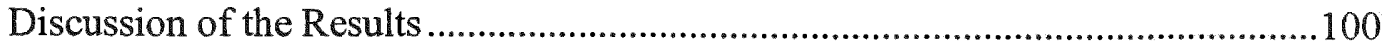

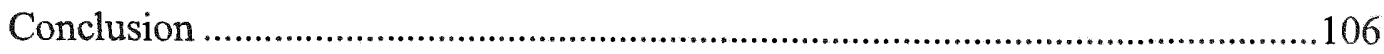

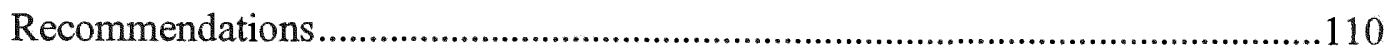

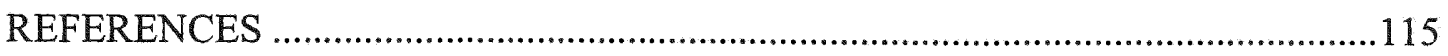

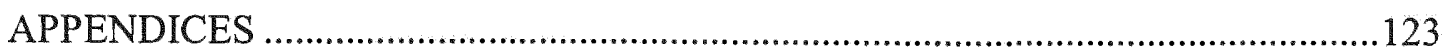

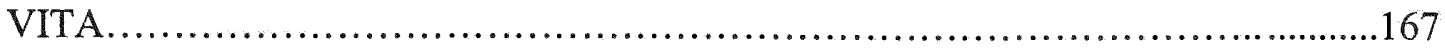




\section{LIST OF TABLES}

TABLE

PAGE

1. Number of Students by Group and Gender. 45

2. Sources of Data and Aspects Analyzed. 62

3. Descriptive Statistics for PSVT Pretest (PreTot) and Posttest (PostTot) 65

4. Test of Homogeneity of Slopes with PostTot as the Dependent Variable. 66

5. Normality Tests for Posttest (PostTot) 66

6. Two-way ANCOVA on PSVT Posttest Score (PostTot).......................................... 67

7. Two-way ANCOVA on Posttest for Participants with Score of 14 or Higher .......... 68

8. Two-way ANCOVA on Posttest for Participants with Score of 13 or Lower........... 68

9. Posttest Estimated Marginal Means for Students with Low Pretest Scores ............. 69

10. Interview Process-Product Scores....................................................................... 74

11. Individual Process-Product Scores and van Hiele Level ......................................... 79

12. Usage of Whole-Part Processes in Protocol and GSP Problems .............................. 80

13. Number of Strategy Types ..................................................................................... 85

14. Number of Strategy Types in which Students Worked Individually........................ 86

15. Number of Complete GSP Constructions (C) and Correct Solutions (S) .................. 87

16. Classroom Usage of Processes and Success Score …................................................ 94

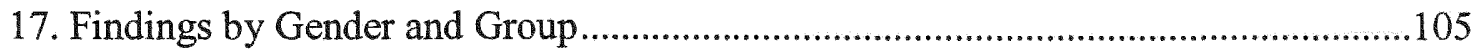




\section{LIST OF FIGURES}

FIGURE

1. Hierarchical relationship of terms................................................................ 5

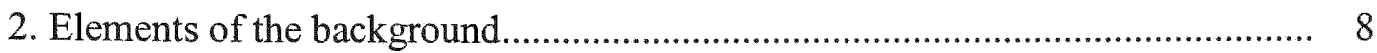

3. Non-linear arrangement of lessons ............................................................ 52

4. Group*gender interaction in low-performance sub-sample .............................. 70

5. Process-product score and mental processes used by students in problem $\# 1$.... 73

6. Diagonals of horizontal and vertical cross-sections ........................................ 76

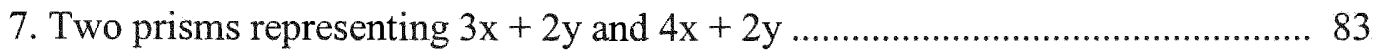

8. Vertical square cross-section projected to lateral face of larger cube ................. 84

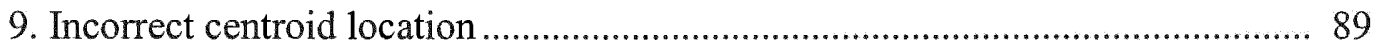

10. Octahedron inscribed in tetrahedron ............................................................. 90

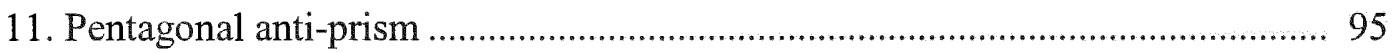

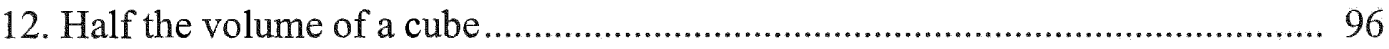

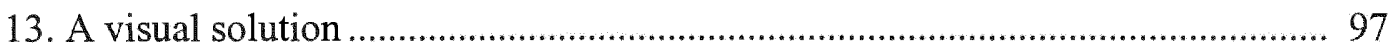

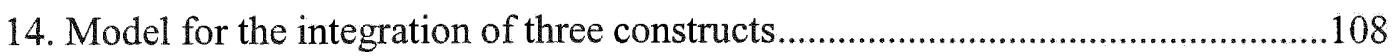

15. Model for the teaching and learning of solid geometry .......................................111

16. Two-dimensional analogy for problem \#1 …...................................................127

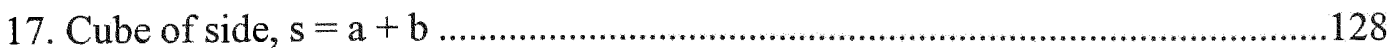

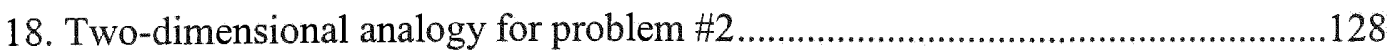

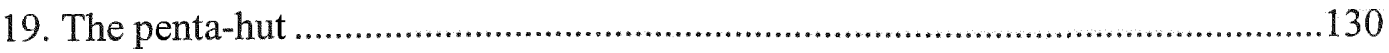




\section{CHAPTER I}

\section{INTRODUCTION}

This study investigates how connecting visual and analytic reasoning through the use of three-dimensional geometric tasks in a constructivist classroom setting affects high school students' spatial visualization abilities. Chapter 1 presents the following sections: background of the problem, statement of the problem, research questions, hypotheses, significance of the study, assumptions of the study, delimitations of the study, definition of terms, and summary. The chapter concludes with a description of the organization of the dissertation.

\section{Background of the Problem}

This section presents two parts to the background of the problem this dissertation addresses. The first part presents the general problem of students' poor geometric understanding in schools in the United States. The second part presents three developments in mathematics education which can be viewed as solutions to the general problem. The author then presents his interpretation of the relationships among the elements of the background of the problem.

\section{General Problem}

Generally, students in the United States receive limited instruction in geometric concepts (Clements, 2003). In grades pre-K through 8 , students typically only learn lowlevel cognitive processes such as identifying figures and their parts (Usiskin, 1982). Consequently, there is weakness in geometric understanding which is evidenced when students are asked to express relationships among the parts of figures (Kaput, 1987). Although students may learn to use formulas relating variables, they do so in an 
algorithmic manner and with no real understanding (Schoenfeld, 2002). Similarly, only $30 \%$ of students can write proofs or exhibit any understanding of their meaning at the end of the high school geometry course (Senk, 1985). Moreover, the topic of spatial properties of solids typically appears only in one chapter of geometry textbooks. This limited coverage results in textbook exercises presented in a shallow and formulaic manner. The outcomes of the Third International Mathematics and Science Study (TIMSS) confirm the pervasiveness of a situation in the United States in which students throughout all grades are introduced to limited basic geometry concepts. The results of this international study showed that elementary school students in the United States do not learn much geometry from grade to grade (Mullis et al., 1997). Specifically, secondary school students scored at or near the bottom in every geometry task of the TIMSS evaluation (Lappan, 1999).

\section{Three Developments Providing Solutions to the General Problem}

Several developments in mathematics education contribute to students' understanding of mathematics in general, and can serve to mitigate the current deficiency in the teaching and learning of geometry. Three developments pertaining to the background of the current study are presented below. The first is the continuing formation and utilization of theories related to learning mathematics. The second development is the integration of mathematics educational tools into the classroom. Finally, the third is the enrichment of the school mathematics curriculum with tasks focusing on the development of spatial abilities and strategies.

Theories of learning mathematics. Since the 1980s, the National Council of Teachers of Mathematics (NCTM) has given recommendations for the improvement of 
student understanding of mathematical concepts and processes (NCTM, 1989). One such recommendation is that the use of multiple representations of knowledge in the classroom facilitates and strengthens the learning process by providing several mutually referring sources of information (NCTM, 2000). Learning guided by the theory of multiple representations allows students to construct understandings that better prepare them for knowledge transfer (Goldin, 2003). A cognitive model proposed by a group of researchers has suggested that visual reasoning and analytic reasoning can and should be integrated (Zazkis, Dubinsky, \& Dautermann, 1996). In the model, the mutual support between visual and analytic reasoning allows a student to more readily narrow down to a correct solution. There is little research on this model beyond a few studies in undergraduate college classrooms (Dubinsky, Dautermann, Leron, \& Zazkis, 1994; Zazkis et al., 1996). Although the authors of this cognitive model recommended investigating the interaction of visual and analytic modes of reasoning in high school mathematics, they did not specify a particular topic in the curriculum (Zazkis et al., 1996).

NCTM has developed five principles which describe features of high-quality mathematics education. Its learning principle states that "students must learn mathematics with understanding, actively building new knowledge from experience and prior knowledge" (NCTM, 2000, p. 20). This principle further recommends that learning with understanding can be enhanced by classroom interactions in which students talk about their strategies to improve problem solving, reasoning, and argumentation.

A constructivist classroom setting as proposed by Clements and Battista (1990) reflects the learning principle proposed by NCTM. According to constructivist theory, 
learning in the classroom takes place in a social context and is mediated by language and socially negotiated understandings between teacher and student (von Glasersfeld, 1996). Learning tasks are designed to offer the students an experiential basis for the abstraction of mathematical concepts, and to provide the opportunity for students to discuss and present their findings (von Glasersfeld, 1996).

Educational tools in the classroom. Integrating educational tools into the classroom in a manner which contributes to students' understanding of mathematics is complex. In general, the use of any tool should be supported by at least one theory of learning (Papert, 1993). This study looks at the use of two educational tools for teaching and learning mathematics: technology and manipulatives.

In the pioneering Soviet studies on integrating spatial and mathematical tasks, not only were students asked to turn, flip, and in any other manner transform solids for the sake of studying their spatial abilities, but students were asked to act mathematically on geometric solids (Kilpatrick \& Wirszup, 1978). Most of the actions were based on measurement, proportion, and the use of three-dimensional coordinate systems. Today, these mathematical actions can be enhanced with the assistance of technology. However, research suggests that one of the main unresolved issues in the integration of technology into the curriculum is how technology should be used in the classroom. Incorporating educational tools such as Geometer's Sketchpad (GSP), a computer program for the dynamic exploration of geometric concepts, may strengthen students' understanding of the subject (Dreyfus, 1990).

Tools such as models of geometric solids provide a visual context within which analytic procedures can be applied and can become concrete and meaningful to the 
student. Although only one form of knowledge--iconic, enactive, or symbolic--is predominant while solving a problem, students usually use all three simultaneously (Bruner, 1960). This study simultaneously incorporates GSP, an iconic form, along with manipulatives, an enactive form of representing knowledge.

Spatial abilities and strategies. The term spatial visualization is used in this dissertation according to a definition given in the context of mathematics education. As shown in Figure 1, mathematics educators have identified visual processing ability as a type of spatial ability (Bishop, 1983). In turn, visual processing ability has been separated into two categories. The present study refers to the category herein labeled spatial visualization ability, which consists of mentally manipulating and transforming visual

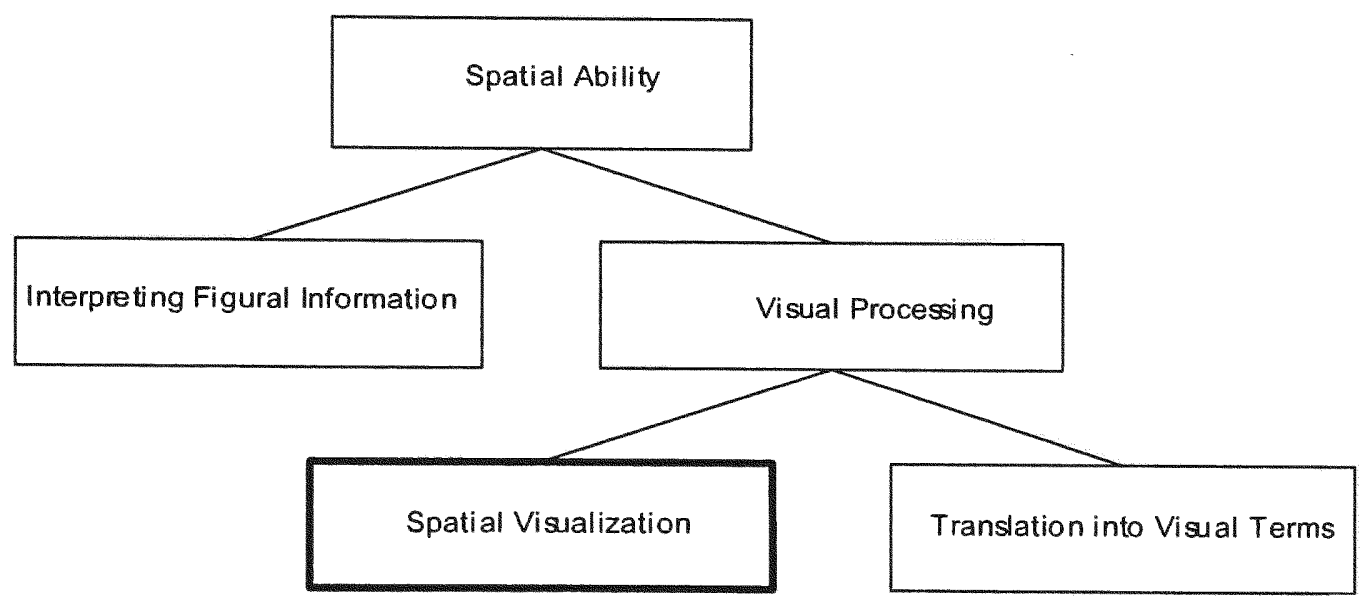

Figure 1. Hierarchical relationship of terms.

representations. Although important, the other categories shown in Figure 1, interpreting figural information and the translation of non-figural information into visual terms, are not addressed in this study. 
Similarly, the term strategy is used to describe a part of the solving process of a mathematical task. Specifically, the term visual processing strategy refers to the use of mental images as an essential part of the solution. Through the analysis of students' visual-analytic strategies rather than focusing on individual preference of cognitive style, math educators may better assist students to reason geometrically (Hershkowitz, 1990). In addition, when students share their solution strategies in the classroom, others learn to create their own (Gorgorio, 1998).

Both visualization abilities and strategies can be utilized to favor students' mathematical understanding. Enriching the curriculum with tasks to develop students' visualization abilities presents an opportunity for meaningful geometric understanding. NCTM recommended developing students' spatial abilities throughout the geometry curriculum (NCTM, 2000). By designing tasks which call for the manipulation of solids, a visual context is provided for the abstract and analytic procedures of mathematics. Similarly, understanding can be improved when students share solution strategies with their peers, especially if done in a constructivist setting. Classroom interactions in which students talk about their strategies to solve problems has improved mathematical understanding (NCTM, 2000). However, the outcomes from research on both students' abilities and strategies are inconclusive and contradictory. As evidenced in the next two paragraphs, whether schooling has an effect on and gender differences exist in spatial ability are two research questions which remain unanswered.

The Soviet studies on the development of spatial abilities showed that training in the school had a significant effect (Kilpatrick \& Wirszup, 1978). However, Lean (as cited in Bishop,1989) reported that visual processing is not trainable. Others have shown that 
spatial abilities can be developed through instruction (Bishop, 1989). In the same sense that students learn to count, there is a natural order to how students can develop spatial visualization abilities (Baartmans \& Sorby, 1996a).

Similarly, differences between males and females in spatial ability are widely disputed as reported in results from meta-analyses (Linn \& Peterson, 1985). Some researchers believe that just providing educational tools, such as manipulatives or computer environments, to both males and females could equally stimulate the process of visualization in both sexes (Bishop, 1983). Others have reported a consistent gap between males' and females' performance in tasks that require spatial ability, with males typically outperforming females (Ansell \& Doerr, 2000).

Schematic relationship among the elements of the background. As shown in Figure 2, the author has developed a schematic representation of the three sections of the background of this study. The outer box circumscribing the two smaller boxes represents how constructivist theory and connecting visual and analytic reasoning serve as a framework for both the teaching and learning of three-dimensional geometry and research on spatial visualization. Firstly, the figure depicts a high school geometry classroom in which three-dimensional geometry is taught and learned with the assistance of educational tools such as computers and manipulatives. In such a setting, and with the aid of manipulatives and computers, students can explore tasks which encourage the utilization of both visual and analytic reasoning. Secondly, the figure portrays how data collected in a constructivist classroom setting can be analyzed quantitatively to measure students' visualization abilities. Additionally, qualitative data can be analyzed to describe the visualization strategies which students use when solving geometric tasks. 
Specifically, the research can focus on three-dimensional visualization strategies which show how students connect visual and analytic reasoning. Finally, Figure 2 shows how the geometry classroom box is connected to the spatial visualization box, showing a direct link between the teaching and learning of school mathematics and research on

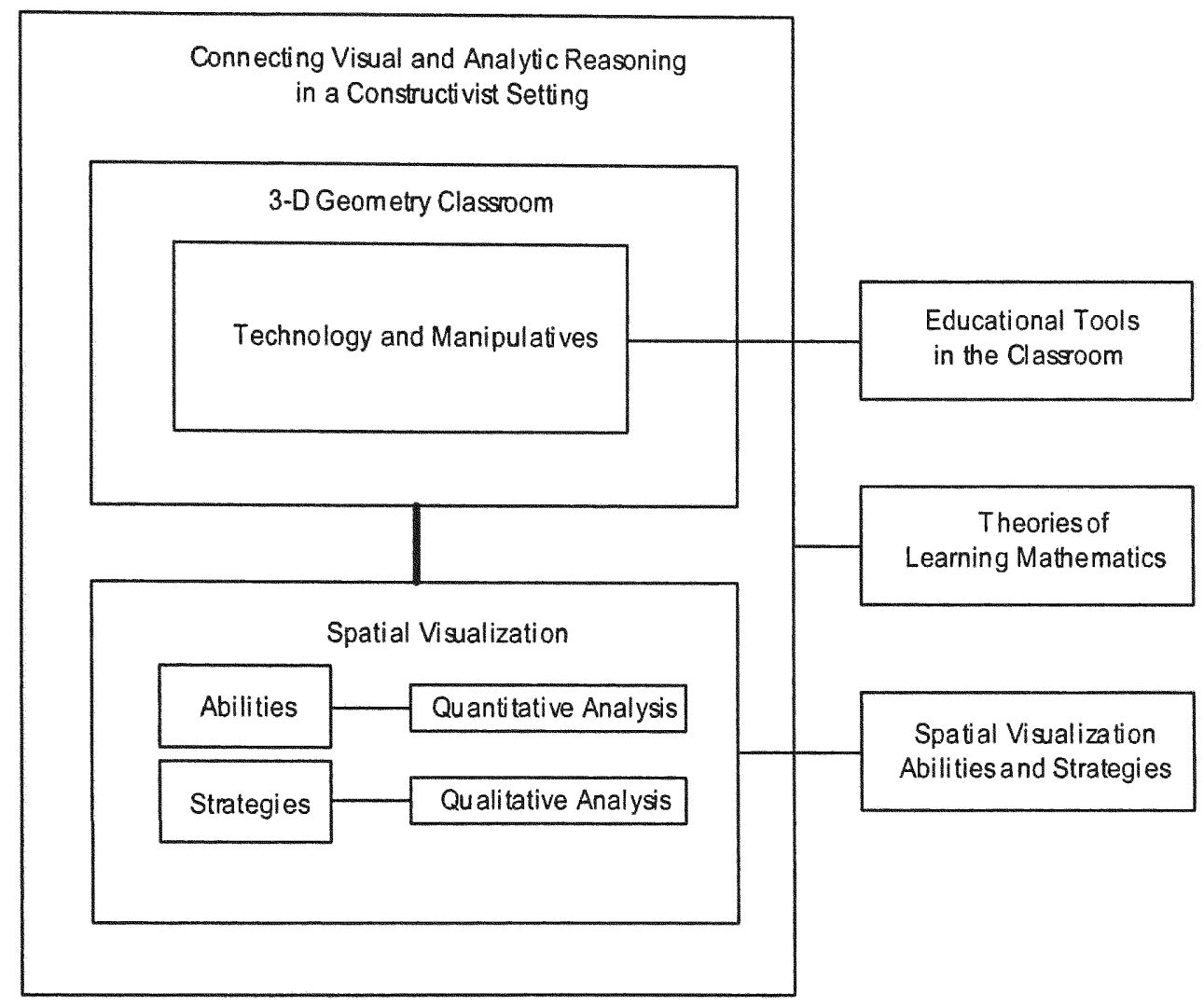

Figure 2. Elements of the background.

spatial visualization. This link is important because it depicts the essence of the present study, namely conducting research on spatial visualization in an actual classroom. The link also represents the existing gap between research and practice. As posed by Amit 
and Fried (2002), the only manner in which reform efforts could be achieved is if researchers go out into the field and document what they find.

\section{Statement of the Problem}

This dissertation investigated whether spatial visualization abilities are affected by students' use of tasks which encourage them to connect visual and analytic reasoning. In addition, the study explored how students used visual-analytic processing strategies as part of the solution of three-dimensional tasks.

\section{Research Questions}

The first two research questions were answered with quantitative data, whereas the third question used qualitative data. The questions were addressed in the context of research conducted in a real classroom setting, and were related to connecting visual and analytic reasoning. Three components present only in the experimental groups facilitated the process of connecting both reasoning modes, namely the use of a constructivist setting, GSP, and manipulatives.

1. Does connecting visual and analytic reasoning to solve three-dimensional geometric tasks affect students' spatial visualization abilities as compared to a control group?

2. Does connecting visual and analytic reasoning to solve three-dimensional geometric tasks affect students' spatial visualization abilities differently:

a. In a group of students who fully construct solids using GSP versus a group of students who partially construct solids using GSP?

b. In males versus females? 
3. How do students in the experimental groups use visual-analytic strategies when solving three-dimensional geometric tasks?

a. What are the differences between students who fully construct solids using GSP and those who partially construct solids using GSP?

b. What are the differences between males and females?

Hypotheses

The following null and alternative hypotheses correspond to the first two research questions:

Null hypothesis 1: There will be no difference in spatial visualization abilities between the groups constructing solids using GSP and the control group.

Alternative hypothesis 1: One group will improve more than the others in spatial visualization abilities.

Null hypothesis $2 \mathrm{a}$ : There will be no difference in spatial visualization abilities between the group fully constructing solids using GSP and the group partially constructing solids using GSP.

Alternative hypothesis 2a: The group fully constructing solids with GSP will outperform the group partially constructing solids with GSP in spatial visualization abilities.

Null hypothesis $2 \mathrm{~b}$ : There will be no difference in spatial visualization abilities between males and females.

Alternative hypothesis $2 \mathrm{~b}$ : Males will outperform females in spatial visualization abilities. 
The researcher expected that the students in the group fully constructing solids with GSP would become aware of all the underlying geometric relationships in their constructions, thereby affecting their spatial visualization abilities more than for the students in the group partially constructing solids with GSP. The researcher also expected both males and females will benefit from the treatment based on the assumption that the 6-week treatment is of a duration sufficiently long to develop males' and females' spatial abilities.

The third research question was answered using qualitative methods. The qualitative research paradigm is characterized by naturally emerging theories from the analysis of the data and, therefore, hypotheses are not presupposed.

\section{Significance of the Study}

Connecting visual and analytic reasoning to improve students' spatial visualization abilities is significant for several reasons. Firstly, there are few studies about the connections of the two modes of thinking which recognize the importance of using visual reasoning along with analytic reasoning (Arcavi, 2003; Gorgorio, 1998; Zazkis et al., 1996). Traditionally, research on improving spatial ability has concentrated solely on students' visual reasoning, thus ignoring the contribution of students' analytic reasoning to the development of their spatial abilities (Gorgorio, 1998). However, analytic processes such as justifying spatial patterns and relationships help students see more than the visually obvious features in a spatial task. Secondly, there is paucity of research on the visual-analytic model, especially as it relates to how students coordinate both modes of reasoning in high school (Zazkis et al., 1996). Moreover, the study is significant because it describes the visual-analytic processing strategies students use when solving 
tasks in three-dimensional geometry. Little has been published regarding the analysis of the strategies present in the solving processes of geometrical tasks taking into account the use of visual or non-visual processing strategies (Gorgorio, 1998). Therefore, there is the need to know more about when particular strategies are important and for what tasks they are important. Finally, as a result of improving students' ability to visualize, their ability to understand and solve geometric tasks may also improve.

Assumptions of the Study

The study uses the following assumptions:

1. The Purdue Spatial Visualization Test (PSVT) was used to collect the quantitative data of this study. This test is assumed to measure gestalt processing, the key cognitive component of spatial ability (Guay, 1977). The PSVT is different from many spatial tests which require a minimal amount of gestalt processing and a significant amount of analytic processing.

2. Changes in students' spatial visualization abilities can be detected in 6 weeks.

3. The systematic narrowing of focus in the qualitative analysis of this study reaches theoretical saturation.

\section{Delimitations of the Study}

This study has the following delimitations:

1. The generalizability of the results is limited to the sample of participants in this study since randomization was precluded.

2. The generalizability of the results is limited to constructivist settings as defined in this study. 
3. Teachers implementing the treatment of this study needed a training session to familiarize themselves with the curriculum tasks and a constructivist setting.

4. The same teacher taught the experimental and control groups.

5. The present study investigated only one of the two visual processes identified by Bishop (1983). Spatial visualization ability, which consists of mentally manipulating and transforming visual representations is investigated, whereas the visualization and transformation of non-figural information into visual terms is not.

\section{Definition of Terms}

Definitions of important terms in this dissertation are presented in this section. Spatial visualization ability. Ability is a skill in a particular domain which can be taught or perfected if innately present. Spatial visualization ability is the ability to mentally restructure or manipulate the image of an object, either by transforming it as a whole (e.g., rotating a solid) or by transforming any of its parts, such as faces, edges, or vertices (e.g., folding a two-dimensional net into a three-dimensional solid). It includes viewing an object from different perspectives (e.g., drawing the isometric view of an object). The construct of spatial visualization ability is measured in this study with the Purdue Spatial Visualization Tests.

Visual processing ability. Visual processing includes spatial visualization. It is also an ability that involves the translation of abstract relationships and non-figural information into visual terms. It is an ability of process, and does not relate to the form of the stimulus material presented (Bishop, 1983). 
Visual-analytic processing strategy. Visual-analytic processing strategies consist of the use of visual images as an essential part of the solving process of a task.

Visual reasoning. An act of visualization or visual reasoning is an act in which an individual establishes a strong connection between an internal construct and something to which access is gained through the senses. An act of visualization may consist of any mental construction of objects or processes that an individual associates with objects or events perceived by her or him as external (Zazkis et al., 1996).

Analytic reasoning. An act of analysis or analytic reasoning is any mental manipulation of objects or processes with or without the aid of symbols. When the symbols are taken to be markers for mental objects and manipulated entirely in terms of their meaning or according to syntax rules, then the act is one of analysis. Analytic reasoning includes the construction of mental images based entirely on other mental images in the absence of external media (Zazkis et al., 1996).

Solid geometry. Solid geometry in this dissertation refers to the study of properties of polyhedra, three-dimensional objects bounded by polygonal faces. Vertices, edges, and faces are the basic elements of solid geometry that permit the study of features like altitude, surface area, and volume.

Dynamic geometry. Dynamic geometry is the capability of software to provide motion of geometric objects in a way that objects contract and dilate without losing their relational properties. In pedagogical explorations of geometry, students experience the dynamic feature of software like GSP when animating and dragging parts of the figures they construct. 
Construction. A construction in this study is a series of mathematically related steps needed to create a dynamic computer model of a three-dimensional object. Students developed dynamic GSP constructions of various Platonic solids using a technique which involved the construction of points rotating around an ellipse and the use of parallel horizontal cross-sections. A limitation of this technique is that the constructions do not rotate about all three axes. Although tilt and vertical-axis rotations are possible, the heights of the objects dilate and contract without proportionally altering the volumes.

Fully constructing solids. One of two modes of using GSP in the present study. The students in one experimental group fully construct their solids if they start their constructions from a blank sketchpad, and proceed to complete them.

Partially constructing solids. One of two modes of using GSP in the present study. The students in one experimental group partially construct their solids if they start their constructions from a template provided by the researcher, and proceed to complete them.

Teaching experiment. An experiment that comprises three main characteristics. It should be, firstly, of a long-term interaction between researchers and students. Secondly, it should focus on how students do mathematics, thereby emphasizing the thinking processes students form and use. Thirdly, it should use a qualitative methodology and analysis of the data collected (Cobb \& Steffe, 1983).

Constructivist setting. Current reform efforts in mathematics education share the basic tenets of constructivist theory as articulated by von Glasersfeld (1995). Based on the position of von Glasersfeld, Clements and Battista (1990) proposed the following five elements of a constructivist classroom setting: 
1. Knowledge is actively created or invented by the child.

2. Children create new mathematical knowledge by reflecting on their physical and mental actions.

3. No one true reality exists, only individual interpretations of the world."

4. Learning is a social process in which children grow into the intellectual life of those around them.

5. When a teacher demands that students use set mathematical methods, the sense-making activity of students is seriously curtailed.

\section{Summary}

Students usually start their high school geometry course relying on low-level cognitive processes (Usiskin, 1982). One way of encouraging students to use higher-level processes is to introduce them to multiple representations of knowledge, which facilitate and strengthen their learning of mathematics. The visual-analytic model of learning mathematics is one example that makes use of multiple representations of knowledge. Connecting visual and analytic reasoning allows students to more readily narrow down to a correct solution (Zazkis et al., 1996).

This dissertation investigated how high school students' spatial visualization abilities are affected through the use of three-dimensional geometric tasks that connect visual and analytic reasoning. Integrating three-dimensional tasks into a real classroom setting in a manner which facilitates the connections of the two modes of reasoning is complex. Three main components which make possible the connections are the use of a constructivist setting, GSP, and manipulatives. 
The study posed quantitative research questions about the effect of connecting visual and analytic reasoning on students' spatial visualization abilities. Two ways of using GSP and whether or not there are differences in gender were investigated. The results may shed light on how GSP could be used better in the classroom. Additionally, using qualitative methods, the study provides a description of visual-analytic processing strategies students use when solving three-dimensional geometric tasks. Answers to these questions are significant for mathematics educators because through the connections of visual and analytic reasoning, students are better equipped to improve their understanding of mathematical concepts.

The next chapters are organized as follows. Chapter 2 reviews previous work related to the research conducted in this dissertation. Areas to be covered include spatial abilities, visualization, and the use of dynamic geometry software. Chapter 3 discusses the research design of the study. Next, chapter 4 presents the results. Finally, chapter 5 discusses and interprets the results to recommend future research directions. 


\section{CHAPTER II}

\section{REVIEW OF THE LITERATURE}

This chapter consists of a review of the literature on the learning and teaching of geometry, specifically in the area of spatial abilities, strategies, and visualization. First, the chapter presents information on two theories of learning mathematics which support this study: constructivist theory and multiple representations theory. It then examines the literature on spatial ability and visualization, thus providing the context for the study. Subsequently, the chapter presents the role of the Geometer's Sketchpad as an educational tool used to develop students' visualization abilities. Finally, the chapter ends with a summary of topics.

\section{Theoretical Framework}

There is a clear distinction between theories and frameworks, as used in this study. Learning theories explain all the factors involved as an individual grows in knowledge and understanding, whereas frameworks guide the researcher in investigating certain aspects of learning. Specifically, frameworks are based on, and can possibly extend, an existing theory of learning (Cottrill, 2003). This study is based on constructivist and representation theories of learning mathematics. However, the teaching experiment is the framework under which the collection and interpretation of this study's data are made (Cobb \& Steffe, 1983).

\section{Constructivist Theory}

As defined by von Galsersfeld (1995), constructivist theory is based on the claim that a person's knowledge is constructed by her or himself. The foundations of this theory are described below according to the contribution of three of its proponents, namely Jean 
Piaget, Lev Vygotsky, and Jerome Bruner. The formalization of constructivist theory, as articulated by Ernst von Glasersfeld, is then presented.

Piaget is considered the pioneer of constructivism due to his description of the process of cognitive epigenesis. During this process, the learner establishes equilibrium through the mechanisms of accommodation and assimilation. According to this theory, a student can progress from one level of understanding to a higher level through reflective abstraction (as cited in Cottrill, 2003). According to the Piagetian school of thought, instruction is subordinate to the specific stages in the development of the child's thinking. The claim is that stages of development manifest at certain age levels and are relatively independent of the conditions of instruction (Kilpatrick \& Wirszup, 1978).

One of the tenets of constructivist theory is that learning is a social process. As recommended by NCTM, integrating the advances of constructivist theory to the classroom facilitates learning (NCTM, 2000). By the use of speech and cooperative activity in the classroom, a student learns to build cognitive tools over which he or she has conscious control. Through this process in which language and thought are related, the role of the teacher is to convey the relationship between the signifier and the signified (Vygotsky, 1978). As a result of an effective role of the teacher, the student internalizes external knowledge (Vygotsky, 1986).

Contrary to the Piagetian relation of each stage of development to a specific period of childhood, simultaneous modes of knowledge classification are claimed by others (Bruner, 1960). Specifically, this theory of cognitive learning emphasizes the formation of a hierarchical system of categorization which facilitates transfer, enhances retention, and increases problem-solving and motivation. Bruner saw three modes, with 
one as dominant during the development of the child. The modes of knowledge classification are the enactive, iconic, and symbolic modes. An example of the enactive mode is evidenced with a child learning to roll over, sit up, or walk. Another example of this mode and based on research on helping students move from van Hiele level 1, suggests that teachers supplement textbook presentations with manipulative materials and examples in the environment (Fuys, 1988). By using the iconic mode, children learn to understand what pictures and diagrams represent, or how to do arithmetic using numbers but without counting. Finally, learners use the symbolic mode when, for example, they engage in the interpretation of algebraic notation.

Additionally, Bruner advocated the discovery-oriented learning methods in schools and introduced the notion of the spiral curriculum (Bruner, 1960). Bruner believed that all topics, in some form or level, must be introduced at an early age, but cannot be exhausted at any age. Thus the learner can always return to the topic to study it with increasing depth. Moreover, and contrary to the Piagetian school of thought, he postulated that any subject can be taught effectively in some intellectually honest form to any child at any stage of development.

In the 1980s, von Glasersfeld advanced an epistemology of constructivism. Current reform efforts in mathematics education such as NCTM's share the basic tenets of constructivism (NCTM, 2000). The idea that there is no way of knowing an absolute reality was put forward by von Glasersfeld (1995). Knowledge from this perspective is constructed from the individual's perceptions and interactions with the world. Knowing is based on previously constructed knowledge and is active, adaptive, and personal. Based on this position, Clements and Battista (1990) proposed five important elements of a 
constructivist classroom setting which affect the way people learn and teach mathematics, and which guided the present study:

1. Knowledge is actively created or invented by the child.

2. Children create new mathematical knowledge by reflecting on their physical and mental actions.

3. No one true reality exists, only individual interpretations of the world.

4. Learning is a social process in which children grow into the intellectual life of those around them.

5. When a teacher demands that students use set mathematical methods, the sense-making activity of students is seriously curtailed.

Elements of constructivism such as the ones listed above are generally incorporated in a teaching framework in one of two ways (Cottrill, 2003). The framework can be built for the purpose of providing a description of students' understandings which correspond to different levels of thought. Another framework may focus on describing how students move from one level to the next, as the researchers look for cognitive mechanisms of knowledge internalization. This study uses a framework that focuses on the former, and presents a description of students' visualization strategies. There are different frameworks used in other studies, such as those based on clinical interviews conducted in laboratory settings. In either the teaching experiment or the laboratory setting, theories of learning support the framework of choice.

\section{Multiple Representations Theory}

A representation is a configuration of signs, characters, icons, or objects that can stand for something else. The term represent can be interpreted in many ways, including 
the following: correspond to, denote, depict, embody, encode, evoke, label, mean, produce, refer to, suggest, or symbolize (Goldin, 2003). This type of definition has been referred to as abstract (Kaput, 1998). To make the definition concrete requires specifying what kinds of entities are involved in representing and being represented, and in what ways one entity stands for another. Kaput (1987) maintained that the concept of representation involved the following components: a representational entity; the entity that it represents; particular aspects of the representational entity; particular aspects of the entity it represents; and finally, the correspondence between the two entities.

The difficulty in articulating an accurate definition for the term representation has led researchers to use the term inscription, which evokes the learner's internalization of external knowledge (Presmeg, 2006). Since researchers cannot directly observe students' inscriptions, inferences are made based on the production of, or interaction with, representations external to the students. The internal representations may be perceptions, visualizations, or imagined objects or symbols (Goldin, 2003). Individual representational configurations, whether mathematical or not, rarely stand alone. Thus, it is essential to consider the notion of a representational system. Internal systems of representation include the natural language of individuals; their visual imagery and spatial, tactile, and kinesthetic representation; their problem-solving heuristics and strategies; and their personal capabilities, including conceptions and misconceptions in relation to conventional mathematical notations and configurations (Goldin, 2003). Five types of systems of internal representation are: verbal/syntactic systems of natural language; imagistic systems of visual-spatial, auditory-rhythmic, and tactile-kinesthetic encoding; formal notational systems of mathematics; systems of planning, monitoring, and 
executive control which guide problem solving; and affective systems that includes not only general, global affect but changing states of feeling or emotion during problem solving. External representation systems include normative natural languages; conventional graphical and formal notational systems of mathematics; and structured learning environments that may include concrete manipulative materials or computerbased micro-worlds (Goldin, 2003).

Internal representational systems are not carbon copies of external systems. Rather, the multiple encodings and the redundancy provided by several different internal representation systems can account for the persistence of schemata in long-term memory, for individuals' ability to reconstruct temporarily forgotten mathematical concepts, and for the longevity of individuals' belief systems in relation to mathematics (Goldin, 2003). Mathematical concepts are learned powerfully when a variety of appropriate internal representations, with appropriate relationships among them, have been developed. Teaching about standardized representations as ends in themselves may fail to develop students' mathematical power. One place where mathematical power of an individual resides is in the individual's ability to translate processes among representational modes, both external and internal (Goldin, 1987).

Empirical research on representation in mathematics education involves observations of individual students' interaction with external representations or their production of such representations. Three distinctions describing features of media important to the interaction of external mathematical representations with internal representations are dynamic versus static media, interactive versus inert media, and recording versus non-recording media (Goldin \& Kaput, 1996). Complex behaviors and 
patterns of behavior can be observed as the individual interacts with the structured external representational environment or in the production of new external representations. After the observations, inferences about students' internal cognitive representation follow. A study was conducted with primary school students solving spatial problems which were intended to evoke visual imagery. The authors of the study noted instances of concrete imagery, dynamic imagery, pattern imagery, action imagery, and procedural imagery (Owens \& Clements, 1988).

\section{Teaching Experiment}

The concept of a teaching experiment as used in this study is in accordance to the definition of problematique. Balacheff (1990) defined problematique as a set of research questions related to a specific theory of learning. In addition, the teaching experiment is used as a framework which guides the researcher in the investigation of a phenomenon, namely students' visualization strategies to solve three-dimensional problems. In this study, the framework provides a context for the research questions. Thus, the research questions are relevant, not only to the theories of learning, but specifically, to an experimental framework organized around students' use of cognitive processes in the classroom.

This epistemological framework also takes into account the role of the teacher in the classroom and the social aspect of mathematics. Pedagogically, it proposes the use of a didactical process (a constructivist setting in this dissertation study) which places the student in a variety of mathematical situations. These include situations in which students explore, conjecture, generalize, validate, formulate, decide, and communicate. The key to 
this framework is to bring the student to epistemological obstacles which he or she must overcome to construct his or her own mathematical knowledge (Artigue, 1992).

The teaching experiment has its origins in the work of Vygotsky and influenced research in the former Soviet Union. The purpose of a teaching experiment is for researchers to experience student's mathematical learning and reasoning. Essentially, a teaching experiment consists of a series of teaching episodes and interviews. Studies that adopt the concept of a teaching experiment offer a new perspective on the complexity of the learning process within its natural setting (Cobb, 2000). Similarly, the goal of the teaching experiment is to provide a research base for the modification of instruction and/or curriculum.

Finally, Cobb and Steffe (1983) outline three main characteristics of the teaching experiment. It should be comprised, firstly, of a long-term interaction between researchers and students with duration between 6 weeks and 2 years. Secondly, it should focus on how students do mathematics, thereby emphasizing the thinking processes students form and use. Thirdly, it should use a qualitative methodology and analysis of the data collected.

\section{Visualization}

Visualization is an important component in several areas of knowing. Different groups of researchers with diverse goals study this field with interest. Because visualization can synthesize an enormous amount of information in a very efficient way, the cognitive processes associated with it become very attractive to researchers. Visualization researchers give strong predictive validity to this construct in estimating success of individuals in the fields of engineering, science, drafting, and design 
(Baartmans \& Sorby, 1996a). In the research literature of mathematics education, visualization is a construct that interacts with the ideas of imagery, spatial ability, and intuition (Bishop, 1983).

Definitions of the Term Visualization

Visualization may be termed as visual perception, spatial perception, spatial imagery, or spatial vision. In the study of three-dimensional solids, the terms generally used are spatial visualization (Gutierrez, Jaime, \& Fortuny, (1991). For these authors, the central and basic element in all conceptions of visual perception is the mental image, a mental representation that people make of physical objects, relations, or concepts. Presmeg (1986) describes five types of mental images: concrete imagery (a picture in the mind); pattern imagery (a pure relationship stripped of concrete details); memory images of formulae; kinesthetic imagery (for example, the physical movement of walking as represented with fingers); and dynamic imagery (the movement or transformation of the image itself).

Kruteskii (1976) used the terms visual imagery. According to him, among other positive qualities associated with visual imagery are its integrative power, its exemplary use, its concretization of abstract ideas, and its sometimes illuminative aspect. For him, the schemes used by students are a unique synthesis of concrete and abstract. Actually, Kruteskii (1976) reported that children have little difficulty in generating visual images. These images are to the students the bearers of sense and content of abstract concepts. However, caution must be exercised not to cause the effect of geometric rigidity, as reported in the literature $(\mathrm{Hoz}, 1981)$. For Hoz, geometric rigidity is the difficulty experienced by a child when unable to see a diagram in a different way. Similarly, the 
term habituation is used when the visualization becomes a symbol for an object (Bishop, 1983).

Because the term visualization has been used in various ways in the research literature of the past three decades, it is necessary to clarify how it is used in this study. Presmeg (2006) defines visualization following Piaget and Inhelder's position that when a person creates a spatial arrangement there is an image in the person's mind, guiding the creation. Thus, for her visualization is taken to include processes of constructing and transforming both mental imagery and all of the representations of a spatial nature that may be implicated in doing mathematics. This characterization is broad enough to include two aspects of spatial thinking elaborated by Bishop (1983), namely, visual processing (VP) and interpreting figural information (IFI). Despite the use of the term visualization in his definition of visual processing given below, Bishop does recognize two distinctive aspects of the term visualization. The first is the noun of visualization, which directs our attention to the product, the object, the what of visualization. The second is the verb of visualization, which on the other hand, makes us attend to the process, the activity, the how of visualization. In addition to those two aspects of visualization, Bishop summarizes that the literature establishes a third aspect of visualization as the consequence of the first two. This third aspect is one that considers how the educational process relates to the first two aspects: the objects and processes of visualization. This third aspect is specifically addressed by the teaching experiment framework of this study.

Bishop (1983) uses the term visualization in defining the ability for visual processing (VP). For him VP is "the ability that involves visualization and the translation 
of abstract relationships and non-figural information into visual terms. VP also includes the "manipulation and transformation of visual representations and visual imagery" (p.183). Rotating a polyhedron in one's mind is an example for the second part of VP. For the particular case of referring to three-dimensional tasks, the term spatial processing ability appears in the literature (Gorgorio, 1998).

The ability for interpreting figural information (IFI) is defined by Bishop as the ability that involves understanding the visual representations and spatial vocabulary used in geometric work, graphs, charts, and diagrams of all types. An example of IFI is interpreting dashed marks representing hidden faces of a solid. Mathematics abounds with such forms, and IFI concerns the reading, understanding, and interpreting of such information. Again, for the particular case of coping with spatial tasks, the term interpreting spatial information appears in the literature (Gorgorio, 1998). In summary, VP is an ability of process and does not relate to the form of the stimulus. For example, visual processing can be the same for either verbal or physical stimuli. On the contrary, IFI is an ability of content and does relate to the form of the visual stimulus, the figure to be interpreted.

The IFI-VP dichotomy relates to the definition by McGee (1979). He identified two types of spatial ability. Spatial visualization (Vz) "involves the ability to mentally manipulate, rotate, twist, or invert a pictorially presented stimulus object." (p. 210). Spatial orientation (SR-O) "involves the comprehension of the arrangement of elements within a visual stimulus pattern and the aptitude to remain unconfused by the changing orientations in which a spatial configuration may be presented." (p. 210). IFI extends SR$\mathrm{O}$ by including geometric and graphical conventions not generally employed in SR-O 
tests, and sharpens it by emphasizing the interpretation demanded by those representations. VP has much in common with Vz, but it extends and sharpens Vz by emphasizing the process aspect rather than the form of the stimulus. Relating to developmental theory, it may well be that there is an optimal time for such exercises in developing VP ability. Bishop believes the optimal age bracket is between 7 and 12 years of age, when children begin to grasp projective space ideas (Bishop, 1983).

\section{Visual-Analytic Reasoning}

As many domains of experience as are relevant should be implicated in any mathematical process at hand (Bishop, 1989). For instance, to increase the level of understanding of a problem, visual and analytic representations of it are recommended Drawing from both brain hemispheres, the analytic left and the visual right, students who make visual-analytic connections have more power to understand a mathematical situation. Indeed, a visual-analytic model to study the cognitive switches between visual and analytic reasoning was proposed by a group of researchers (Zazkis et al., 1996). They observed that the two modes of reasoning are mutually dependent. While a student is working on a mathematical task, visualization enriches analysis and analysis supports visualization. In this mutually dependent process, a student develops a deeper understanding of the task at hand.

Furthermore, there is evidence that geometrical concept attainment is, at least partially, a result of logical-analytical features (Hershkowitz, 1990). By using the analytic mode, features of solids are recognized, spatial patterns are discovered, and spatial relationships are established and justified —all of which help the student see more than the obvious in a spatial task. Thus, analytic reasoning provides a logical justification for 
enriching visual transformations. It gives the necessary support to enhance visual imagery and hence improves spatial abilities (Zazkis et al., 1996). Similarly, there is evidence of the facilitative and general beneficial effects of using computer-generated visual images in developing students' understanding of algebraic ideas (Bishop, 1989).

The interaction of visualization with the idea of intuition is presented in the research literature of mathematics education. In solving a problem, a student's initial intuitions may be faulty. Therefore, they should not be trusted, and it is only the symbolic argument, or analytic solution, that the student ends trusting (Papert, 1980). Visualization can be thought of as a way of resolving conflict between correct symbolic solutions and incorrect intuitions (Arcavi, 2003). It is also a way that helps students reengage with and recover conceptual underpinnings which may be easily bypassed in formal, analytic solutions.

Finally, there is a clearly identifiable, but unconventional movement developing in the mathematics community, whose aim is to make visual reasoning an acceptable practice of mathematics, alongside and in combination with algebraic reasoning. Visualization should be understood as a support and illustration of essentially symbolic results, and possibly providing a proof in its own right (Arcavi, 2003).

\section{Ongoing Theory Development}

In a historical review by Presmeg (2006), research on visualization in the 1980 s focused on teaching that promoted effective mathematical visualization. During the 1990s, because visualization research came to be recognized as a significant field for mathematics education, some studies incorporated aspects of curriculum development, and particular content areas were investigated. As with the intersecting field of spatial 
ability, gender differences in visualization were also a topic of interest. The effect of gender on visualization is addressed in the next section of this chapter, namely spatial abilities and strategies. Other important questions were investigated, including the seeming reluctance of students to visualize in mathematics. Moreover, the influence of technology, particularly in dynamic computer environments, was explored and continues to be a significant focus. The 2000 s are seeing a broadening of the focus on visualization to include semiotic theories. Even research on the use of gesture in meaningful learning of mathematics has begun to take on a significant role.

The need to know if there exist links between visualization and processes of abstraction and generalization is noted in recent papers (Pitta-Pantazi, Gray, \& Christou, 2004; White \& Mitchelmore, 2003). Another ongoing and important theme is how visualization interacts with the didactics of mathematics. Effective pedagogy that can enhance the use and power of visualization in mathematics education is perhaps the most pressing research concern today (Woolner, 2004). Very few studies have addressed this topic since Presmeg (2006) reported the results of her study of classroom aspects that facilitate visualization. Presmeg proposed a list of 13 questions that appear to be of major significance for research on visualization in mathematics education. Of those, four are relevant to this study.

1. What aspects of pedagogy are significant in promoting the strengths and obviating the difficulties of use of visualization in learning mathematics?

2. How can teachers help learners to make connections between visual and symbolic inscriptions of the same mathematical notions? 
3. How can teachers help learners to make connections between idiosyncratic visual imagery and inscriptions, and conventional mathematical processes and notations?

4. How do visual aspects of computer technology change the dynamics of the learning of mathematics?

These research interests demonstrate the need for ongoing theory development. The need for overarching theories that could unify the whole field of visualization in mathematics education was recognized and is receiving attention (Gutierrez, 1996). Purdue Spatial Visualization Test

The Purdue Spatial Visualization Test (PSVT) is a battery of tests used as an instrument to collect quantitative data on students' spatial visualization abilities (Guay, 1977). The test is divided in three parts with multiple-choice questions about the visualization of rotations, views, and developments. Each question refers to representations of a solid which is either rotated, viewed from various perspectives, or whose faces are unfolded onto a flat surface.

The reliability and validity of the PSVT are understood by answering two questions. The first one is about reliability: Are similar results obtained when the test is given to similar populations? The second one is about validity: Is the test a valid measure of the construct it claims to measure? In a study at Purdue University, the first step toward estimating the reliability of the rotations test involved calculating the KuderRichardson 20 (KR-20) and/or split half (SH) reliability coefficients (Bodner \& Guay, 1997). The computation of these coefficients suggested that the test was internally consistent. These data were obtained in studies of more than 4800 students enrolled in 
general chemistry or organic chemistry courses for either agriculture and health science or science and engineering majors at Purdue University. The average of the KR-20 and/or SH reliability coefficients reported from six studies was 0.81 . Evidence for the reliability of the test can also be obtained from the mean and standard deviation data for different populations, thus reflecting the precision of the test. As cited in Bodner and Guay (1997), researches obtained virtually identical means and standard deviations when testing different groups of students at Purdue University. One study reported a mean of 13.84 and a standard deviation of 3.84 , compared to another one with a mean of 13.96 and a standard deviation of 3.80. A 20-item version of the rotations test was administered to 1273 and 1648 students, respectively. To assure construct validity, a 30-item version of the rotations test was used as one of five measures of spatial ability in a study of the relative importance of cultural and neurophysiological factors in spatial test performance (as cited in Bodner \& Guay, 1997). The two most highly correlated spatial ability scores were on the PSVT rotations test and on the Shepard-Metzler test $(r=0.61, p<0.001)$.

\section{Spatial Abilities and Strategies}

In the 1920s, researchers considered mechanical aptitude tests as unreliable measures of general intelligence. Due to the general acceptance of spatial abilities, these tests became known as spatial ability tests. A debate rapidly began among researchers about the number and identities of spatial factors. Results of studies using factor analysis eventually reduced them to two major factors: spatial orientation and spatial visualization (Bodner \& Guay, 1997). Researchers continue to debate whether spatial abilities can be taught and whether there are gender differences. The following two sections present a diversity of results in these areas. Contradicting the findings of other psychological 
studies, Sexton (1992) concluded that instruction can have an effect on spatial visualization abilities. As there is no clear relationship today between gender and spatial visualization ability, gender is usually considered a potential source of confounding in many studies (Alias, Black, \& Gray, 2002).

Schooling Effect

Several studies have claimed spatial visualization skills cannot be taught effectively through typical instructional methods (Salkind, 1976). Lean (as cited in Bishop, 1983) did not find any evidence showing the success of training in visual processing. However, an equal number of studies have found that spatial visualization can be improved through instruction (Gillespie, 1995). Contradicting the findings on many psychological studies, Sexton (1992) concluded that it is indeed possible to improve spatial visualization skills if the instruction is appropriate and the delivery time sufficiently long. Additionally, other researchers think it is clear that encouragement to develop visual images and to promote general visualization awareness is attainable through teaching (Baartmans \& Sorby, 1996b). What type of experience and the length of the experience have yet to be firmly established (Strong \& Smith, 2002).

It appears that providing diverse spatial activities may be the key to enhancing overall spatial visualization ability (Baartmans \& Sorby, 1996b). Baartmans and Sorby prescribed diverse spatial activities to their engineering students, ranging from manipulation of concrete models to computer visualization activities, and found that they improved in their spatial visualization ability as measured by the Purdue Spatial Visualization Test. Similarly, research shows that visualization is a very personal matter (Bishop, 1983). Therefore, slower students need to be given the time to develop their own 
images. Identical images do not result in different students when such a personal process takes place. Possibly due to this personal nature of visualization, some studies fail to find any evidence showing the success of training in visual processing (Bishop, 1983).

In the United States, the National Council of Teachers of Mathematics (NCTM) stresses the importance of developing students' spatial abilities throughout the geometry curriculum. In contrast to this belief, the emphasis of Piaget's followers was upon the natural course of development, leaving aside the influence of the school. Soviet psychologists, on the other hand, rejected this position. They contended that spatial concepts and spatial abilities are best studied as they develop under the influence of schooling. However, contrary to the Piagetian notion of sequential developmental stages, the schooling effect may exercise influence on the sequence and the very character of the stages (Kilpatrick \& Wirszup, 1978).

A series of Soviet investigations on the development of spatial ability in a school setting focused on several aspects of mathematical learning (Kilpatrick \& Wirszup, 1978). Below are listed some of the aspects that are relevant to the treatment of the present study:

1. The formation of mathematical concepts and the related questions of generalization, abstraction, and concretization.

2. The mental operations of analysis and synthesis.

3. The development of spatial perception.

4. The development of logical reasoning.

5. The structure and special features of mathematical abilities. 
Some of these studies presented difficulties students had in drawing plane sections of solids and the tendency to use calculations rather than graphic constructions to solve problems. Also, in some studies, it was shown that the knowledge of the rules for representing three-dimensional figures is a prerequisite for instruction in solid geometry. Moreover, the Soviet studies showed that accurate measurement depends upon an understanding of perspective, which in turn depends on an adequate notion of space (Kilpatrick \& Wirszup, 1978).

The construct of strategies is useful for explaining students' solving processes in school mathematics. As a construct for analysis, the term strategies has a different character from the constructs of ability or preferred processing mode. Math educators may better understand the way students visualize in three dimensions through the analysis of group strategies rather than focusing on individual preference of cognitive style (Gorgorio, 1998). The analysis has the purpose of exploring two aspects in terms of the task characteristics which stimulate students to use different strategies. One aspect is the categorization of the different strategies used. The other aspect is the effectiveness of particular strategies to solve tasks of specific characteristics. In addition, from the point of view of teaching, strategies can be shared and therefore taught, while preferred processing mode is an individual's trait. Different tasks will stimulate different images and processes in an individual. Specifically, ease or difficulty of the tasks is one important feature of the stimulus context. Easier tasks led visualizers away from visual methods (Paivio, 1991). 
Gender Effect

In addition to the controversy of the effect of schooling in the development of spatial abilities, gender differences appear in the literature as another source of disagreement between researchers. In the last 30 years, a consistent gap was found between males' and females' performance in tasks that require spatial ability, with males typically outscoring females (Ansell \& Doerr, 2000). For example, two specific studies reported males outperforming females (Allen \& Hogeland, 1978; Eisenberg \& McGinty, 1977). In addition, a study found that roughly three-quarters of the males scored at or above the female mean, and in most studies the difference between the male and female means was larger than two-thirds of the standard deviation of the scores (as cited in Bodner \& Guay, 1997).

Despite the better performance of males noted, earlier research suggested that sex differences in spatial ability resulted from differences in the way spatial tasks are processed rather than from differences in spatial ability itself (Yen, 1975). There is general agreement that two major processing strategies are used to solve spatial tasks: analytic processing versus gestalt processing. Gestalt processing occurs when an individual forms and transforms visual images in an organized whole in much the same way that one recognizes faces of people. The distance between the eyes or the color of their eyes is secondary when remembering a face as a whole. Analytic processing occurs when the whole is broken into individual parts, whose relationship is mapped in a one-toone process.

In addition to the way spatial tasks are processed, childhood experiences rather than gender may cause differences between males and females. Mitchelmore's study (as 
cited in Bishop, 1983) addressed the aspects of the environment which interact in some way with visualization. For example, he interpreted the relatively weak spatial and visualizing skills among his learners in Jamaica as a consequence of having few toys. Many homes he studied lacked special play equipment for children. Just providing educational material, such as manipulatives or computer environments to both males and females could encourage the creation of visualizations, and thus the visualization process itself in both sexes (Bishop, 1983). Among male subjects, Deno (1995) found nonacademic activities such as model building, sketching, and assembly of parts during the high school years were correlated to spatial visualization. Among females however, this relationship was not statistically significant. Males who had played with Lego toys in preschool and elementary school also scored significantly higher on spatial visualization tests.

Others found that spatial ability differences do exist between sexes but the differences depended upon the students' field of study. For example, Vandenberg and Kuse (1978) and Hamilton (1995) found that males perform better than females on mental rotation tasks only. Still others believe differences disappear after instruction on spatial skills. Ben-Chaim et al. (1988) found that only prior to instruction, males in middle school were better in their spatial visualization ability compared to females. Finally, in a study with 21 year-old college students, the differences in spatial ability between the means for female and male groups were not significant (Alias, Black, \& Gray, 2002). Participants in this study used building blocks as aids to visualization and sketched objects constructed from blocks, both from observation and imagination. 


\section{The Geometer's Sketchpad}

The Geometer's Sketchpad (GSP) is an important software development in the field of learning and teaching mathematics which has affected the way geometry is taught in schools (Jackiw, 1995). GSP offers its users the possibility of constructing dynamic figures which preserve their mathematical relationships after being dragged with the computer mouse. Teachers can take advantage of this capability by encouraging students to make conjectures about the properties of their constructed figures. With an effective pedagogy such as the constructivist setting of the present study, students can engage in open-ended explorations of relationships of geometric figures rather than in the memorization and repetition of procedures for proving theorems. Textbooks such as Discovering Geometry: An Inductive Approach (Serra, 1993), encourage students to create their own geometric constructions with GSP and to formulate the mathematics which describes the relationships students discover.

Research on how to use GSP shows three ways in which the software program can be utilized (Bennett, 1999). The first way is using pre-made sketches to focus on the mathematical relationships of existing figures rather than on creating constructions. The second way is integrating different mathematics topics in a geometric construction rather than using the linear presentation typical of school textbooks. In a single triangle investigation, for example, students might explore side and angle relationships, area, transformation, symmetry, and coordinate geometry. The organizational scheme chosen by the teacher does not dictate a proper order in which to present the topics. Instead, a network of connections between topics can be used by the teacher to discover students' needs of curiosity, level of understanding, and feelings of ownership. The third way is 
having students drag parts of the figures during explorations to experience the dynamic nature of the software. By dragging the parts, figures contract and dilate without losing their relational properties. This capability of GSP may help develop students' visual processing abilities.

Studies with computer-generated images also show that dynamic visual images when properly employed by educators can develop students' spatial visualization abilities. The computer becomes a tool for the mental manipulation of graphical representations which can provide informative feedback to the user (Dreyfus, 1990). The immediate visual feedback provided by these dynamic images can lead to a cognitive conflict, and thus can provoke the student to rethink a problem's solution process and the geometrical analysis of the situation (Hershkowitz, 1990). Additionally, recent research on how users of GSP take meaning from an image which is moving, has shown that focused attention is needed to notice change. Manipulation of specific objects on the screen in ways that assist students to see the objects as representatives of a class of objects or a class of constructions with invariant properties is important. From this basis, students are able to generalize and reflect upon geometric properties (Rensink, 2000).

As an extension of the first way of using GSP presented by Bennett (1999), it is important to note that Sinclair (2004) used the term preconstructed instead of pre-made. Preconstructed sketches created with GSP, as well as preconstructed, web-based sketches can be used as an alternative to having students make their own constructions. These preconstructed sketches can be prepared by a teacher, and should include action buttons to move and to animate objects in order to facilitate students' investigations. Students creating their own mathematical diagrams are aware of the underlying relationships the 
diagram attempts to present. If, on the other hand, the students have to interpret someone else's diagram, they have no prior knowledge of the underlying relationships and must rely on the diagram, the context, and their visual skills for an accurate interpretation (Sinclair, 2004).

The three ways of using GSP presented by Bennett (1999) are supported by the line of research conducted by the Dutch mathematics educators Pierre van Hiele and Dina van Hiele-Geldof because they allow for the individual's development of geometric thinking levels (van Hiele, 1986; van Hiele-Geldof, 1984). The van Hieles learned that students pass through a series of levels of geometric thinking while learning geometry: visualization, analysis, informal deduction, formal deduction, and abstract geometric axiom systems (van Hiele, 1986). Similarly, when using three-dimensional GSP constructions, these ways of using GSP are supported by the idea that spatial structuring is an important process in students' construction of geometric knowledge (Battista, 1999). Finally, it is also possible that appropriate educational activities using GSP can facilitate the transition from one level to another in the van Hiele theory of geometric thinking (July, 2001). When constructing three-dimensional objects in GSP, students must attend to the structural relationships of the physical prototypes in order to construct appropriate computer models. In particular, students must attend to the arrangement of the edges, faces, and vertices of the solid and their positions relative to each other (July, 2001).

\section{Summary}

This chapter first presented the theoretical framework of the study on students' spatial visualization abilities and strategies. Constructivist theory and representation theory are two theories of learning mathematics which support this study. The distinction 
between theories and frameworks was made to establish that learning theories explain all the factors involved as an individual grows in knowledge and understanding, whereas frameworks guide the researcher in investigating certain aspects of learning.

The teaching experiment supported by constructivist theory and representation theory provides an epistemological framework for the present study. Within this framework, students construct knowledge when placed by the teacher in situations which allow for the movement from one level of understanding to a higher level through reflective abstraction. Learning takes place in the relationship between the student representing and interpreting mathematics, and in the shared meaning with others. Through the collection and analysis of data, the role of the researcher is to provide descriptions of the visual and analytic strategies students use while solving threedimensional tasks.

As presented in this literature review, there is an interaction between spatial abilities, strategies, and visualization. The interaction specifically appears in terms of the role of the school in their development. Additionally, the chapter presented that through the connections of visual and analytic reasoning, students' can narrow down to correct solutions as explained by the visual-analytic model. Finally, the chapter presented how the computer program Geometer's Sketchpad can be used as a tool to develop students' geometric thinking and spatial visualization abilities. 


\section{CHAPTER III}

\section{METHODS}

This study uses a methodological approach for the analysis of data as described by Miles and Huberman (1984). These authors' methodology can be characterized as one in which the qualitative analysis is performed by partially quantifying the data. The qualitative analysis has the purpose of describing how students in two experimental groups used visual-analytic processing strategies to solve three-dimensional tasks. This description brings light to the results of a quantitative analysis which compares pretest to posttest data on spatial visualization abilities for each participant. Therefore, as posed by Greene et al. (1989), one purpose of using mixed methods is to provide complementarity. These authors highlight complementarity as a purpose used to clarify and illustrate results from one method with the use of another method. In such an approach the researcher examines overlapping and different facets of a phenomenon.

This chapter explains how three $10^{\text {th }}$ grade geometry classes participated in the study during 6 weeks. Descriptions of the subjects, setting, materials, treatment procedures, and instruments used to collect data are presented. The chapter ends with a presentation of the methods of analysis, both quantitative and qualitative.

\section{Research Design}

Three major implications from the review of the literature were drawn for structuring the research design of the present study. First, the connections that students make between visual and analytic representations of the same mathematical notions may improve their visualization and mathematical skills. Second, a distinction between ways in which the computer can be used in the classroom may shed light into how technology 
can be used more effectively for the improvement of visualization and mathematical skills. Finally, a third implication is that as there is no clear relationship today in the literature between gender and spatial visualization ability, gender could be a potential source of confounding in statistical analyses.

The present study used a quasi-experimental design for the quantitative comparison of three groups' spatial visualization abilities. The term quasi-experimental refers to experiments that have treatments and outcome measures, but do not use random assignment (Cook \& Campbell, 1979). Since the study took place in a school setting where the students had already been assigned to classrooms, random assignment of students to the three groups was not possible. This situation resulted in a nonequivalent comparison-group design. However, to counteract the effect of non-equivalence of groups, a quantitative analysis of covariance on students' spatial visualization abilities was performed. The design which refers to the quantitative component of this study consisted of administering to a control and two experimental groups a pretest and posttest before and after the treatment, using the pretest as the covariate. This component of the study tested the hypothesis that connecting visual and analytic reasoning will better improve students' spatial visualization abilities as compared to instruction that makes little or no use of the connection of the two. Gender and two ways of using GSP were explored as two causes that may affect the outcome of the results.

Additionally, the research design considered a second component which used qualitative methods. Four sources of qualitative data were collected for the students in the experimental groups. Data from interviews, field notes, GSP files, and protocol problems were analyzed to provide a description of students' visual-analytic processing strategies 
while solving three-dimensional tasks. These sources of data are further described in the section on methods of analysis presented below. Appendix G presents a brief description of two pilot studies which were conducted to familiarize the researcher with the data collection process and the implementation of a curriculum based on visualization tasks. The pilot studies were conducted with the purpose of developing appropriate forms for the qualitative data gathering process (see Appendix C), and to test the validity of the learning tasks in each of the six lessons used in the treatment (see Appendix A).

\section{Subjects}

The study was conducted in an urban school district in Miami-Dade County, Florida. The public high school selected for the study offers three geometry courses based mainly on student achievement on the annual state examination. Students in this school are tracked into informal geometry, regular geometry, or honors geometry. A total of 79 participants in three $10^{\text {th }}$ grade classes of regular geometry participated in the study. Both pretest and posttest data were collected for only 64 students. Table 1 shows these students arranged by group and gender. The students did not have prior experience with GSP.

\section{Table 1}

Number of Students by Group and Gender

\begin{tabular}{lccc}
\hline \multirow{2}{*}{ Group } & \multicolumn{2}{c}{ Gender } & Total \\
\cline { 2 - 3 } & Males & Females & \\
\hline Control & 8 & 13 & 21 \\
Experimental 1 & 10 & 12 & 22 \\
Experimental 2 & 9 & 12 & 21 \\
Total & 27 & 37 & 64 \\
\hline
\end{tabular}




\section{Setting}

The classes in the school where the study was conducted met in block schedule, with even periods meeting for 100 minutes on even days of the month. Students in the sixth period were assigned to the control group; those in the second period, to the first experimental group; and those in the fourth period, to the second experimental group.

\section{Control Group}

The control group was not provided with the tasks connecting visual and analytic reasoning. Students in this group followed the curriculum which had been used since the beginning of the school year. Similarly, the teacher continued using his pedagogical style. The students in this group spent $80 \%$ of the time working with the Cognitive Tutor (2004), a computer-based geometry curriculum in which students use one computer per student to advance through predetermined lessons.

The topics in the curriculum include properties of right triangles, parallelism and perpendicularity, transformations, similarity, quadrilaterals, three-dimensionality, and theorems for circles. The students in the control group remained in their seats, but were allowed to discuss solutions to the problems with their neighbors. If they had questions, students raised their hands and the teacher would assist them. The other $20 \%$ of the time was used for testing and debriefing of homework. There were instances during this time in which the teacher would briefly explain a concept by writing on the board. A teachercentered approach of transmitting knowledge to the students was used most of the time.

Since the study occurred during the last 9-week grading period of the school year, some students in this group had finished the scheduled chapters of the Cognitive Tutor curriculum, and thus were assigned by the teacher to work on review chapters. During the 
third and fourth weeks of the study, all students in this group revisited the Cognitive Tutor's chapter on surface area and volume of solids. The computer environment in which these topics of solid geometry were portrayed to the students contained figures of solids as seen on a regular geometry textbook. All figures were static two-dimensional representations of solids appearing on the computer screen as they would on a textbook page.

Experimental Groups

The students in the experimental groups were taught by the same teacher in the control group. However, the teacher changed his pedagogical style to one following the elements of constructivist theory as presented in the previous chapters of this dissertation. The difference between the experimental groups and the control group was that the experimental groups were provided with the tasks connecting visual and analytic reasoning. The students in these groups used GSP instead of the Cognitive Tutor, which they had used in the first three 9-week periods of the year. Additionally, students in these experimental groups used the Polydron manipulatives as support material for the exploration of the learning tasks. These groups spent $50 \%$ of the time using GSP and $50 \%$ of the time using manipulatives. Every student had a computer and access to enough manipulatives to use as support material for the solution of every problem.

Constructivist class setting. A constructivist setting was created for the two experimental groups by emphasizing group and class discussions. Six groups of four or five students were formed every day. The class started with each group choosing to solve one of several problems available. As soon as a group finished the problem chosen, the teacher would present to the group a new problem related to the first, but with a higher 
level of difficulty. The teacher walked around the class initiating a discussion in each group. The role of the teacher was to encourage students to reflect on their findings. Students' questions and opinions were valued by the teacher, who thus tried to create an environment of trust in which any question was considered important. In this manner, students were able to share their strategies and reasoning processes with their peers. At times, students used their GSP constructions to explain concepts and procedures to their group members. Thus, the teacher acted as a mediator soliciting students' point of view. Rather than the teacher simply telling the students the answer to a problem, he asked the students to use GSP and the manipulatives to start a discussion with their peers. When the teacher probed students with questions, the researcher recorded data on how the students were reasoning and visualizing. Additionally, there was time allocated for group presentations. The teacher assessed each student's learning progress as well as the group's performance and asked questions to the other students listening to the presentations. During these presentations, students displayed the problem statement and solution on a three-by-two-foot erasable whiteboard. While standing in front of the class, the students who were presenting corrected mistakes noted by the audience. The presentations were video taped in case the researcher needed to verify or add detail to his field notes.

Difference between experimental groups. Students in the first experimental group fully constructed solids with GSP, as opposed to students in the second group who partially constructed the solids. A full construction in this study is described as a GSP construction that has been totally created by the student, starting from a blank screen, and therefore requiring a greater procedural intensity. The students in the first experimental 
group, however, were able to use old constructions they had previously fully constructed. A partial construction is described as one in which the student has added elements to an initial and incomplete construction provided by the teacher. The distinction between the two experimental groups can be clarified with examples. For instance, in constructing the dual of a cube, a student fully constructing the dual can open from his or her files a previously fully constructed cube and can proceed to construct the dual by locating the centers of each face of the cube. In contrast, for the same example of constructing the dual of a cube, a student partially constructing the dual, opens a file of a cube showing two segments connecting the centers of two adjacent faces of a cube, and proceeds with the construction of the dual. In another example, when computing the length of the diagonal of a cube, a student partially constructing a solid, opens a file of a cube showing a rectangular cross-section through the diagonal of a face of the cube. The cross-section is highlighted in yellow and pre-designed by the teacher with the purpose of guiding the student or providing a hint for the location of the diagonal of the cube. Hence, the students in the experimental group making partial constructions used a less procedural way of using GSP than the first experimental group.

\section{Materials}

The computer program Geometer's Sketchpad and the Polydron manipulatives were used in the two experimental groups as two major support tools for the learning and teaching of concepts in solid geometry (Polydron International, 2003). A description of GSP is included in chapter 2. The Polydron manipulatives are polygonal-shaped pieces that can be snapped together to create a variety of polyhedra. Equilateral triangles, squares, regular pentagons and hexagons-all of unitary side lengths, rectangles with 
width: length ratio $1: \sqrt{ } 2$, and right isosceles triangles with unitary leg lengths were used to form the solids relevant to this study. Along with these two tools, color blocks were used as alternative manipulatives to the Polydron cubes. Physical models of Platonic solids were made available to students for observation and reference throughout parts of the study. Students were asked to use the manipulatives in the first lesson, but for subsequent lessons all the solids, color blocks, and polygons were made available in a box placed in the middle of the classroom. Students were then allowed to access these materials as needed. Two Polydron kits of about 300 assorted polygons each and 200 color blocks were shared by the students in each class. A particular textbook was not used for the study. Instead, mathematical tasks were designed by the researcher, arranged into six lessons, and field tested in the pilot studies. Tasks for each lesson were given to the students as the study progressed. The following section describes each of the six lessons. These lessons explicitly promoted the visual and analytic reasoning of students, and are available in Appendix A. Complementary problems used during the treatment are found in Appendix B.

\section{Procedure}

After administering the pretest to all students in the three groups, the treatment was applied to the two experimental groups. These two groups had availability to didactical material used to assist students in the exploration of geometric tasks which encouraged students to make connections between visual and analytic reasoning.

\section{Instruments}

Prior to the treatment, the students in all three groups took the PSVT as a pretest which was used as the covariate in the quantitative analysis. Similarly, after the 
treatment, the students took the same test as a posttest. The tests had three parts, each with twelve questions on the visualization of developments (DEV), rotations (ROT), and views (VIE). Participants' aggregate scores on these three parts are measures of their spatial visualization abilities. The participants answered the 36 questions in that order, taking ten minutes for each of the three parts. The validity and reliability of the instrument were presented in chapter 2 of this dissertation.

As for the qualitative analysis, four sources of data were used to collect data for three males and three females in each of the two experimental groups. The sources of data were the researcher's field notes, forms with students' solutions to four protocol problems, student interviews, and electronic files of the GSP constructions made by the students. These sources provided the data necessary for the description of students' visual-analytic processing strategies while solving three-dimensional tasks.

\section{Treatment}

After the pretest was administered to the three groups, students in the experimental groups worked on geometric tasks categorized into six lessons. All six of these lessons can be characterized as including tasks which stimulate students to make connections between analytic and visual reasoning. The students worked on the tasks using a constructivist classroom setting, GSP, and manipulatives-three components that facilitate the use of visual and analytic reasoning.

As the teacher and students engaged in the process of constructing knowledge, the researcher collected data necessary for the qualitative analysis. Following the characteristics of a teaching experiment, the focus was on how students constructed mathematical knowledge, thereby paying close attention to the thinking processes 
students formed and used. In addition, the teaching experiment comprises two other main characteristics as described by Cobb and Steffe (1983). It should be of a long-term interaction between researchers and students, and it should use a qualitative methodology and analysis of the data collected.

Each lesson contained a series of tasks which investigated a particular mathematical topic. Most of the tasks in two of the six lessons were considered independent as shown in Figure 3. Lesson \#1 focused on the use of algebraic expressions to represent three-dimensional features of solids; and lesson \#6 focused on facilitating the solution of three-dimensional problems by using two-dimensional analogies. The tasks on

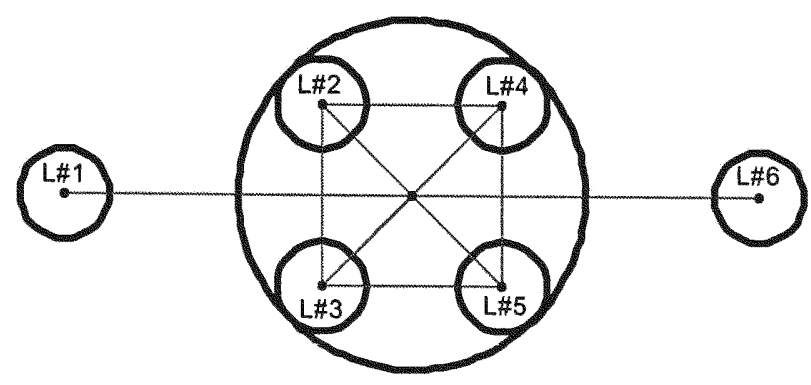

Figure 3. Non-linear arrangement of lessons.

the other four lessons referred mostly to the properties and relationships of duals of Platonic solids and, therefore, were interrelated. Lessons \#2 through \#5 focused on investigations of two dilation theorems; the measurement of length, area, and volume; the construction of GSP models; and the labeling of three-dimensional coordinates of vertices, as pertaining to the duals of Platonic solids. Problems for each of the six topics are presented in Appendix A. The six topics are described below. 
Algebraic expressions. These tasks focus on representing algebraically the geometric structure of solids. Using variables to represent areas and volumes, students readily find connections between visual and analytic reasoning. For example, the surface area of a rectangular prism with three distinct measures for length, width, and height is traditionally expressed as $2 a b+2 b c+2 a c$, where $a, b$, and $c$ are each of the three dimensions. The algebraic expression for the surface area with the procedure used in this study is $2 \mathrm{Q}+2 \mathrm{R}+2 \mathrm{~S}$, where $\mathrm{Q}, \mathrm{R}$, and $\mathrm{S}$ represent each of the three distinct rectangular areas of the solid's faces. This symbolic notation is an analytic form of encoding information relevant to the visual and geometric structure of the solid in reference, namely the six faces of the solid.

Two-dimensional analogies of three-dimensional problems. These tasks focus on constructing two-dimensional representations which facilitate the solution of threedimensional problems. The tasks were presented on the last week of the treatment after the students had been familiarized with the new curriculum and the constructivist classroom setting. Group work and class discussions were emphasized in these tasks. Students' communication of their rationalizations, intuitions, and strategic planning were encouraged rather than mathematical processes leading to the solution. For example, one of the tasks asks for a two-dimensional representation of the structure of a geometric solid constructed from a cube. The solid is described as the volume generated by six intersecting pyramids whose bases are the square faces of the cube.

Duality of platonic solids. These tasks are exclusively investigated using GSP since the duals are nested within each Platonic solid in an arrangement which cannot be 
easily done with manipulatives. Some tasks require that students find proportional ratios between the lengths, areas, and volumes of a Platonic solid and the dual of its dual.

Coordinate geometry. These tasks sought to develop a system of representation for the spatial location of the vertices of geometric solids. Once the students have internalized the system of representation, edge lengths, face areas, and volumes can be computed using the three-dimensional coordinates of the vertices.

Dilation theorems. These tasks investigate a mathematical theorem on dilations. The theorem is first discovered for the two-dimensional case: If two similar polygons have lengths of corresponding sides in the ratio of $m: n$, then their areas are in the ratio of $(m: n)^{2}$. Similarly, the theorem is discovered for the three-dimensional case: If two similar solids have corresponding dimensions in the ratio of $m: n$, then their volumes are in the ratio of $(m: n)^{3}$.

Measurement of length, area, and volume. In these tasks students are encouraged to build models, not exclusively of the five Platonic solids, with the Polydron manipulatives. Measurement of edge lengths is recommended since GSP verification of mathematical computations is not possible for three-dimensional constructions with the current version of the software.

All tasks encourage making connections between visual and analytic reasoning, but most focus on slicing three-dimensional solids and finding mathematical properties and relationships in duals of Platonic solids. Finally, every task includes exercises for individual learning and questions for group discussion. Two important factors for the implementation of the lessons follow: 
Time. Students in the experimental groups spent six of the last 9-week grading period on the treatment. The first and sixth weeks were respectively devoted to investigating the tasks on algebraic expressions and analogies. The intermediate 4 weeks were devoted to tasks from any of the four remaining lessons. Since the students participating in the study did not have previous experience with GSP, they took more time than expected in solving the problems on the first week of the study.

Sequence. The tasks were presented to the students according to their daily cognitive needs. The teacher and researcher met periodically to determine what order of tasks was more appropriate, independent of the lesson to which the tasks belonged. Thus, the tasks were presented in a non-linear sequence. All tasks within a lesson did not need to be completed by the students before they started a task in another lesson. Figure 3 shows that lessons number one and six were the first and last lessons, respectively. The tasks in the four remaining lessons were staggered depending on students' needs and interests.

\section{Methods of Analysis}

Two types of data were collected for this study. Quantitative data in the form of PSVT scores from a pretest and posttest provide measures of students' spatial visualization abilities before and after the treatment. The first component of the study uses a standard quantitative method of analysis, namely an analysis of covariance. This component of the study tested the hypothesis that connecting visual and analytic reasoning will improve students' spatial visualization abilities. The second component uses qualitative data in the form of student interviews, GSP files, solutions to protocol problems, and researcher's field notes. These four sources of data were analyzed to 
provide a description of students' visual-analytic processing strategies while solving three-dimensional tasks.

\section{Quantitative Data}

The data collected for the quantitative analysis of the dependent variable were the PSVT aggregate scores (PreTot and PostTot) obtained by totaling the scores from three tests, namely visualization of rotations (ROT), developments (DEV), and views (VIE). Students scored one point for each correct answer, with the possibility of scoring a maximum of twelve points in each of the three sub-scores. Therefore, a participant could possibly obtain a maximum aggregate score of 36 points. The aggregate score is valid due to the belief that a battery of tests best captures the participants' spatial visualization abilities (Bodner \& Guay, 1997). Pretest and posttest sub-scores for the control and two experimental groups can be found in Appendix E.

The independent variable, connecting visual and analytic reasoning, is encapsulated in a yes-no nominal label used to differentiate the control from the experimental groups. The treatment provided to the two experimental groups consists of a series of tasks which encourage students to make connections between visual and analytic reasoning. In particular, the connections are facilitated by the use of three components in the classroom, namely the use of a constructivist setting, GSP, and manipulatives. Therefore, the control group was coded with a zero, wherein the students did not $(0)$ make explicit connections between the two modes of reasoning. Alternatively, the experimental groups were coded with a one since the students did (1) explicitly connect visual and analytic reasoning. 


\section{Quantitative Method}

The quantitative method of analysis used in this study is a standard statistical procedure known as analysis of covariance (ANCOVA). This procedure combines regression and variance analyses, thus controlling for the effects of the covariate and increasing the precision of the research by reducing error variance. The analysis in the present study examined PSVT posttest performance after adjusting for entering level performance of the participants in the pretest. Using the pretest as the covariate, the procedure investigated the effect of the independent variable (connecting visual and analytic reasoning) on the dependent variable (spatial visualization abilities). Gender and two ways of using GSP are nested in the analysis.

\section{Qualitative Data}

The data collected for the qualitative analysis were student interviews, GSP constructions, solutions to protocol problems, and the researcher's field notes. The qualitative analysis of data in this study drew its methodology from Kantowski's (1974) model for the systematic description of cognitive processes students use in solving geometry problems. The model uses product and process, and sequencing criteria to reduce the collected data of students' solutions to mathematical problems. Modifications to the model were made based on two characteristics of the present dissertation that are not present in Kantowski's study: the teaching experiment framework and the computerbased tasks. Appendix $\mathrm{C}$ shows the forms used to collect the qualitative data.

Protocol problems. The first source of data was the protocol problems. Twelve students working individually recorded with pencil the solutions to four solid geometry problems. No figures were provided with the written statement of the problem (see 
Appendix H). These students were randomly selected, but with one constraint: three males and three females from each of the two experimental groups. Each of the four problems corresponds to one of the first four lessons, namely algebraic expressions, dilation, measurement, and duals of Platonic solids. The form used to record students' responses was divided into four parts in which students could provide: a numerical solution and answer, a statement of at least one mathematical truth used to solve the problem, a sketch, and a written explanation of the problem (see Appendix C). The first two problems were administered after the third week of the treatment, and the two other at the end of the sixth week. The purpose of allocating 3 weeks between the two protocol sessions was to possibly detect an increase in students' use of visual-analytic strategies as a function of time. Appendix F presents how the development of sketching abilities was determined.

Individual students' solutions to these four problems were evaluated with a process-product scoring procedure whose rubric is presented in Appendix D. The rubric was designed to provide a measure of students' visual-analytic processing strategies. In addition to one process point given for each of three processes, only one product point is given for a correct answer. The three processes evaluated were recalling relevant information, using a sketch, and explaining the solution. The process-product score represented how well the student used the three processes which facilitate obtaining a correct answer. Therefore, partial scores between zero and four were possible as shown in Appendix D.

Additionally, a checklist for nine whole-part processes is used to identify the frequency with which students use them while solving the four problems. The nine 
processes are separated into two categories which distinguish how students either perceive solids as a whole or as formed by several parts. Four processes correspond to the traditional mathematical transformations of rotation, reflection, dilation, and translation. These four processes relate to perceiving the solid in question as a whole. The other five processes relate to perceiving the solid as comprised of several parts. These five processes are combining and separating, slicing, folding, connecting, and intersecting.

Interviews. The next source of data is the interviews conducted with the same twelve students who solved the protocol problems. These data provided detailed insight into the cognitive processes students used when solving only one of the four problems. Three questions about visual reasoning were asked first, and three about analytic reasoning followed. The first question related to visual reasoning was: What mental image can you use as a guide in solving this problem? The first question related to analytic reasoning was: What mathematical theorem/formula/relation applies when solving this problem? The other two visual and two analytic questions depended on the participants' response to the first questions.

Three mental processes were checked during the interview: switching from visual to analytic reasoning or vice versa; recalling visual and/or analytic prior knowledge; and using the nine whole-part processes of reflecting, rotating, translating, dilating, combining and separating, folding, slicing, connecting, and intersecting. These processes were recorded as either present or absent during the interview.

GSP files. The third source of data is the GSP constructions created by the 43 students in the two experimental groups. The students constructed three-dimensional models of geometric solids referenced in eight problems which focused on measurement 
and proportion. Four problems on the duals of Platonic solids were selected for the analysis. For each of the four problems, a table is shown in Appendix I which lists different types of GSP strategies used by the students. Each row in the table identifies a type of strategy based on the students' response to the mathematical question posed. A description of either a verbal or numerical explanation is provided. Additionally, scores are presented in chapter 4 , both for completeness of the constructions and for correctness of the solutions. A table listing the frequency with which students used the nine wholepart processes is also presented in the next chapter.

Field notes. Finally, the last source of data is the researcher's field notes. The frequency with which students in the experimental groups used the whole-part processes described above was recorded while they solved class problems. Table 16 in chapter 4 shows the tabulation for 14 class problems (see Appendix J) solved during the last 3 weeks of the study, the time during which the two experimental groups differed. Additionally, success scores reflect the students' ability to explain how a correct answer was obtained. Video recordings for approximately 10 of the 30 hours of the study complemented the field notes. During the analysis, there were instances in which the lack of clarity and detail from the field notes were complemented by observing video clips.

\section{Qualitative Method}

The qualitative method of analysis used in the present study is oriented toward a naturalistic-interpretive paradigm (Glaser \& Strauss, 1967). Within this paradigm, interviews and multiple sources of data are used to capture the perspectives of the participants. In qualitative research, credibility and trustworthiness are analogous to validity and reliability in the quantitative paradigm. Credibility is ensuring that the 
theoretical framework generated is understood and is based on the data from the study. The systematic narrowing of focus and rigorous data analysis guarantee trustworthiness (Rossman \& Rallis, 2003). Finally, a figure representing the theoretical model generated after the analysis is provided as part of the conclusions of the study.

Triangulation of multiple sources of data and the constant comparison method are powerful approaches to achieve trustworthiness (Schoenfeld, 2002). The more independent sources of confirmation there are, the more robust a finding is likely to be. In this study, information from the four sources of data about the phenomenon in question (students' visual-analytic processing strategies) is triangulated by comparing data from different sources to portray a consistent message. Thus, the data are said to converge or reach saturation. The methodology can be characterized as one in which the qualitative analysis is performed by partially quantifying the data (Miles \& Huberman, 1984). In such a manner, tabulations of frequencies, percentages, and graphical representations guide the researcher in making accurate interpretations and providing a robust description of the phenomenon studied.

Table 2 lists aspects within each source of data that allow for triangulating within each source. These aspects emerged as the analysis was conducted, but are based on the observations of several researchers. For example, the process-product score is based on the work of Kantowski (1974); using visual and analytic prior knowledge, and switching between them is based on the visual-analytic model (Zazkis et al., 1996); and tabulating the frequency of the whole-part processes is based on a variety of spatial ability tests (Shepard \& Metzler, 1971; Vandenberg \& Kuse, 1978; Wheatley, 1990). The table also shows the number of data points collected for each source of data. For instance, for the 
interview data source, twelve students were interviewed on one problem. However, for the protocol and GSP problems twelve students provided responses to four problems in each of the two data sources. Finally, 14 field note entries were analyzed. Each of these entries corresponds to one student explaining the solution to a class problem.

Table 2

Sources of Data and Aspects Analyzed

\begin{tabular}{|c|c|c|c|}
\hline Source & $\begin{array}{l}\text { Data } \\
\text { Points }\end{array}$ & $\begin{array}{l}\text { Aspect } \\
\text { No. }\end{array}$ & Aspect Description \\
\hline Interviews & 12 & $\begin{array}{l}1 \\
2 \\
3 \\
4\end{array}$ & $\begin{array}{l}\text { Process-Product Score } \\
\text { Using VA* Prior Knowledge } \\
\text { Switching between VA* } \\
\text { Frequency of Whole-Part Processes }\end{array}$ \\
\hline Protocol Problems & 48 & $\begin{array}{l}1 \\
2\end{array}$ & $\begin{array}{l}\text { Process-Product Score } \\
\text { Frequency of Whole-Part Processes }\end{array}$ \\
\hline GSP Problems & 48 & $\begin{array}{l}1 \\
2 \\
3 \\
4\end{array}$ & $\begin{array}{l}\text { Type of Strategy } \\
\text { Working Individually } \\
\text { Success in Explaining Answer } \\
\text { Frequency of Whole-Part Processes }\end{array}$ \\
\hline Field Notes & 14 & $\begin{array}{l}1 \\
2\end{array}$ & $\begin{array}{l}\text { Success in Explaining Answer } \\
\text { Frequency of Whole-Part Processes }\end{array}$ \\
\hline
\end{tabular}

Note $(*) . V A=$ visual and analytic reasoning.

The outcome of the analysis is presented in the next chapter as a description of how students in the experimental groups form, develop, and utilize visual-analytic processing strategies while immersed in a constructivist setting which promotes students' visual and analytic reasoning. The description represents the diversity of perspectives among the research participants. Its intent is to document students' visual-analytic 
processing strategies with the purpose of identifying effective recommendations for improving students' understanding of three-dimensional geometry. In addition, Table 17 in chapter 5 reports which experimental group and gender was dominant in each of the aspects analyzed.

\section{Summary}

This study tested the quantitative hypothesis that connecting visual and analytic reasoning may better improve students' spatial visualization abilities as compared to instruction that makes little or no use of the connection of the two. Three $10^{\text {th }}$ grade geometry classes participated in a quasi-experimental design with one control group and two experimental groups. The two classes assigned to the experimental groups received a treatment for 6 weeks in which students investigated tasks that encouraged the connections of visual and analytic reasoning. The connections were supported by the use of a constructivist setting, GSP, and manipulatives. A statistical analysis of covariance examined student's spatial visualization abilities as measured by their performance on the Purdue Spatial Visualization Test. The design consisted of giving the three groups a pretest and posttest before and after the treatment, with the pretest used as the covariate. Qualitative data were also analyzed for three males and three females from each of the two experimental groups. The qualitative analysis focused on students' visual-analytic processing strategies, and used triangulation based on the systematic collection of four sources of data: interviews, protocol problems, field notes, and GSP files. 


\section{CHAPTER IV}

\section{ANALYSIS AND RESULTS}

This chapter presents the quantitative and qualitative analyses. For the quantitative analysis, there were two sources of data: the PSVT pretest and posttest. These two sources were analyzed with an ANCOVA which used a five percent level of significance for all tests. For the qualitative analysis, four sources of data were used in this study. The first one was a set of individual interviews in which students explained how to solve a problem. The second source was a set of four protocol problems in which students provided solutions individually. The third one was a set of four GSP constructions in which students worked in groups or individually. Both protocol and GSP problems are presented in Appendix H. Finally, a collection of field notes focusing on how individual students explained the solutions to classroom problems was the fourth source of data. The qualitative analysis was based on the triangulation between and within sources as recommended by Schoenfeld (2002).

\section{Quantitative Analysis}

Preliminary Analysis

Descriptive statistics for the pretest and posttest are presented in this section. Table 3 shows means and standard deviations by group and gender for the PSVT pretest (PreTot) and posttest (PostTot). The scores are respectively labeled with PreTot and PostTot to represent the total score obtained by summing the sub-scores from the three parts of the test, namely the visualizations of rotations, developments, and views. The table shows that the mean spatial visualization ability for each group increased at least 2.5 points from pretest to posttest. 
Table 3

Descriptive Statistics for PSVT Pretest (PreTot) and Posttest (PostTot)

\begin{tabular}{|c|c|c|c|c|c|c|}
\hline \multirow[b]{2}{*}{ Group } & \multirow[b]{2}{*}{ Gender } & \multirow[b]{2}{*}{$n$} & \multicolumn{2}{|c|}{ Pretest } & \multicolumn{2}{|c|}{ Posttest } \\
\hline & & & $\bar{M}$ & $S D$ & $\bar{M}$ & $S D$ \\
\hline \multirow[t]{3}{*}{ Control } & Female & 13 & 13.08 & 5.545 & 14.54 & 4.789 \\
\hline & Male & 8 & 14.38 & 8.618 & 20.00 & 7.746 \\
\hline & Total & 21 & 13.57 & 6.698 & 16.62 & 6.492 \\
\hline \multirow[t]{3}{*}{ Full } & Female & 12 & 13.75 & 6.552 & 15.67 & 7.901 \\
\hline & Male & 10 & 15.30 & 4.877 & 18.50 & 6.187 \\
\hline & Total & 22 & 14.45 & 5.771 & 16.95 & 7.155 \\
\hline \multirow[t]{3}{*}{ Partial } & Female & 12 & 11.83 & 4.407 & 15.25 & 4.957 \\
\hline & Male & 9 & 14.11 & 5.689 & 17.33 & 7.778 \\
\hline & Total & 21 & 12.81 & 4.996 & 16.14 & 6.231 \\
\hline \multirow[t]{3}{*}{ Total } & Female & 37 & 12.89 & 5.471 & 15.14 & 5.870 \\
\hline & Male & 27 & 14.63 & 6.203 & 18.56 & 7.013 \\
\hline & Total & 64 & 13.63 & 5.808 & 16.58 & 6.548 \\
\hline
\end{tabular}

Analysis of Covariance

Before performing the ANCOVA, it was necessary to test the homogeneity-ofslopes assumption. The test evaluates the interaction between the covariate and the factor in the prediction of the dependent variable. A significant interaction between the covariate and the factor would suggest that the differences on the dependent variable among groups vary as a function of the covariate. As desired, Table 4 shows that the interaction is not significant, $F(2,58)=1.2, p=0.239$. 
Table 4

Test of Homogeneity of Slopes with PostTot as the Dependent Variable

\begin{tabular}{lrrrrr}
\hline Source & $S S$ & $d f$ & $M S$ & $F$ & Sig. \\
\hline Group & 46 & 2 & 23 & 1.2 & .299 \\
PreTot & 1611 & 1 & 1611 & 87.1 & .000 \\
Group * PreTot & 54 & 2 & 27 & 1.5 & .239 \\
Error & 1073 & 58 & 18 & & \\
\hline
\end{tabular}

With the assumption of homogeneity of slopes tested, the analysis can proceed after conducting a normality test for posttest (PostTot) scores. The results of two normality tests are displayed in Table 5.

Table 5

Normality Tests for Posttest (PostTot)

\begin{tabular}{ccccccccc}
\hline \multirow{2}{*}{ Source } & \multicolumn{3}{c}{ Kolmogorov-Smirnov } & & \multicolumn{3}{c}{ Shapiro-Wilk } \\
\cline { 2 - 4 } \cline { 6 - 8 } \cline { 6 - 8 } & Statistic & $d f$ & Sig. & & Statistic & $d f$ & Sig. \\
\hline Standard Residual for PostTot & .093 & 64 & .200 & & .980 & 64 & .389 \\
\hline
\end{tabular}

After conforming to the assumptions of homogeneity of slopes and normality, the results of a two-way analysis of covariance indicated a non-significant main effect for 
group, $F(2,57)=0.2, p=0.805$; a significant main effect for gender, $F(1,57)=3.5, p=$ $0.068 / 2=0.034 ;$ and a non-significant interaction of the two factors, $F(2,57)=1.3, p=$ 0.282. Table 6 shows the summary of the ANCOVA.

Table 6

Two-way ANCOVA on PSVT Posttest Score (PostTot)

Partial Eta Observed

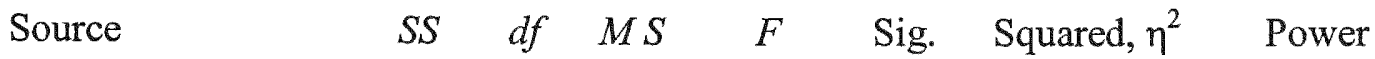

\begin{tabular}{lrrrrrrr}
\hline PreTot (Covariate) & 1460 & 1 & 1460 & 81.6 & .000 & .589 & 1.000 \\
Group & 8 & 2 & 4 & 0.2 & .805 & .008 & .083 \\
Gender & 62 & 1 & 62 & 3.5 & .068 & .057 & .447 \\
Group * Gender & 46 & 2 & 23 & 1.3 & .282 & .043 & .269 \\
Error & 1021 & 57 & 18 & & & & \\
\hline
\end{tabular}

Two-way ANCOVAs were also conducted to explore the treatment effect on the spatial visualization abilities of students in two sub-samples. Students scoring in the pretest below or above the $50^{\text {th }}$ percentile were labeled as low- or high-performing, respectively. Table 7 shows that for the case of 31 participants with a pretest score of 14 or higher, there was a non-significant main effect for group, $F(2,24)=1.3, p=0.287$. Similarly, Table 8 shows that for the case of 33 participants with a pretest score of 13 or 
Table 7

Two-way ANCOVA on Posttest for Participants with Score of 14 or Higher

\begin{tabular}{lccccccc}
\hline & & & & & \multicolumn{5}{c}{ Partial Eta } & Observed \\
Source & $S S$ & $d f$ & $M S$ & $F$ & Sig. & Squared, $\eta^{2}$ & Power \\
\hline PreTot & 409 & 1 & 409 & 18.4 & .000 & .434 & .984 \\
Group & 58 & 2 & 29 & 1.3 & .287 & .099 & .256 \\
Gender & 16 & 1 & 16 & 0.7 & .400 & .030 & .130 \\
Group*Gender & 65 & 2 & 33 & 1.5 & .249 & .109 & .283 \\
Error & 534 & 24 & 22 & & & & \\
\hline
\end{tabular}

Table 8

Two-way ANCOVA on Posttest for Participants with Score of 13 or Lower

\begin{tabular}{lccccccc}
\hline Source & & & & & & Partial Eta & Observed \\
& $S S$ & $d f$ & $M S$ & $F$ & Sig. & Squared, $\eta^{2}$ & Power \\
\hline PreTot & 208 & 1 & 208 & 20.8 & .000 & .445 & .992 \\
Group & 55 & 2 & 27 & 2.7 & .083 & .174 & .493 \\
Gender & 29 & 1 & 29 & 2.9 & .099 & .101 & .378 \\
Group*Gender & 99 & 2 & 50 & 5.0 & .015 & .277 & .763 \\
Error & 259 & 26 & 10 & & & & \\
\hline
\end{tabular}


lower, the main effects for group and gender are not interpretable since there was a significant interaction between the two factors, $F(2,26)=5.0, p=0.015$. The probability of there being a significant difference in group drastically reduced from $p=0.805$ for the total sample to $p=0.083$ for the sub-sample of students entering the study with low pretest scores.

A pairwise comparison followed to identify whether there was significant difference for the effect of group within the effect of each gender, or the effect of gender within the effect of each group. The pairwise comparison analysis was conducted among the estimated marginal means of the posttest, as listed in Table 9. Covariates appearing in the statistical model were evaluated at the pretest mean value of 9.24 . The results showed

Table 9

Posttest Estimated Marginal Means for Students with Low Pretest Scores

\begin{tabular}{lccc}
\hline Gender & Control & Fully Constructing & Partially Constructing \\
\hline Male & 16.37 & 16.60 & 9.96 \\
Female & 12.68 & 11.42 & 13.02 \\
\hline
\end{tabular}

that within males, there was a significant difference between groups, $F(2,26)=5.9, p=$ 0.008. Similarly, there was a significant difference between males and females within the group fully constructing models with GSP, $F(1,26)=6.9, p=0.014$. Specifically, the control group and the group fully constructing models exhibited significantly better scores than the group partially constructing models. Similarly, males scored significantly 
higher than females in the group fully constructing models. The results are represented graphically in Figure 4.

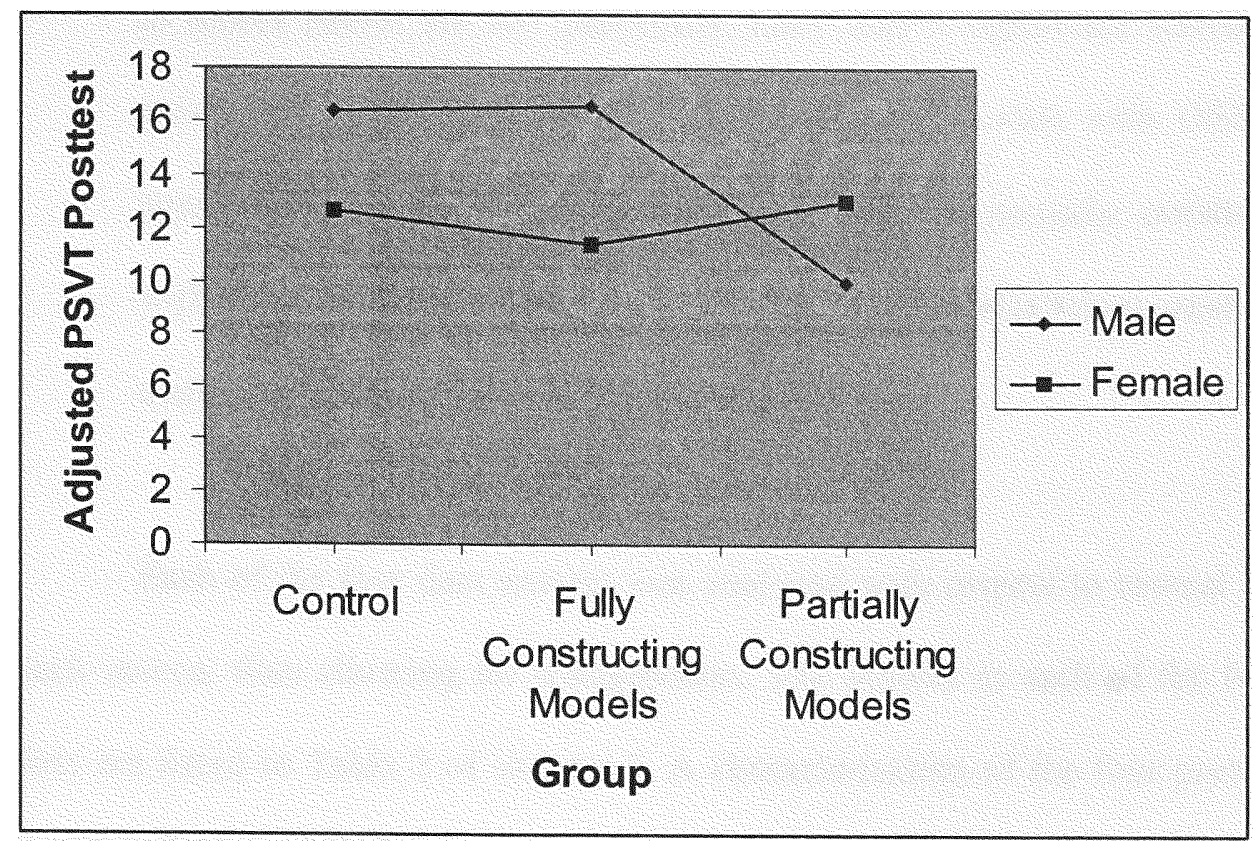

Figure 4. Group*gender interaction in low-performance sub-sample

\section{Summary of Quantitative Results}

These results are from a quasi-experimental design, and cannot be generalized to a broader population. As discussed in chapter 3, the participants were not randomly assigned to the experimental groups due to the school's predetermined scheduling of classes. The quantitative results of two-way ANCOVAs indicated three main findings.

1. There was no significant difference between the mean PSVT posttest scores of the group fully constructing models, the group partially constructing models, and the control group. 
2. There was a significant difference between the mean PSVT posttest scores of males and females, with males performing better than females.

3. In the sub-sample of participants with pretest scores of 13 or lower, equivalent to scores below the $50^{\text {th }}$ percentile, there was a significant interaction between group and gender. Students fully constructing models with GSP significantly benefited more from the treatment than students partially constructing models with GSP. In addition, within the group fully constructing models with GSP, males significantly benefited more than females.

Qualitative Analysis

Each of the four data sources was analyzed with respect to several aspects within each source, thus allowing for triangulation. The aspects in each of the four sources of data are listed in Table 2 of chapter 3. A characterization of the four protocol problems and four GSP problems is presented in this section with the purpose of establishing what strategies are necessary for specific task characteristics.

Interviews

The interviews focused on students' explanations to the problem of finding the height of an octahedron given that its edges measure one unit. The interviews were approximately 20 minutes long and were conducted on the last two days of the fifth week of the study. Four different aspects from the data collected were analyzed. The first aspect analyzed was the process-product score. As shown in Figure 5, four females and two males scored above the median. The figure also shows that the interview median score for the group fully constructing models was higher than the median for the group partially constructing models. 
Figure 5 is a discrete graph showing the process-product score on the vertical axis and student identifiers on the horizontal axis. The student identifiers are numbers one through six for each group, preceded by letters B for boy or $G$ for girl. The red points refer to the score obtained by each student working individually on protocol problem \#1, whereas the red lines indicate the median score for each group (short lines) and for both groups combined (long line). Similarly, the blue points and lines refer to the scores obtained during the interviews. Horizontal and vertical brackets enclosing a point indicate the student used both visual and analytic switching and prior knowledge, respectively.

Finally, a rectangle, a circle, or a triangle around the point indicates the number of nine whole-part processes used by the student during the solution of the problem. The rectangles, circles, and triangles show the student used 0-2 processes, 3-4 processes, or 56 processes, respectively. For example, the girl identified as G4 in group one (fully constructing models with GSP) scored four points. This constitutes a perfect score composed of 1 product point for a correct answer and 3 process points for recalling relevant information, using a mental image, and explaining the solution. The girl identified as G4 also used either five or six of the nine whole-part processes, as indicated by the triangle. Moreover, this girl used both visual and analytic prior knowledge and visual-analytic switching, as indicated by the vertical and horizontal brackets. In general, it can be noted that a low score corresponds to students using fewer processes during the interview, as can be deduced from most rectangles located below the long blue median line.

Most of the process-product score is a reflection of the three process score components, namely ability to recall, manipulate a mental image, and explain a solution 


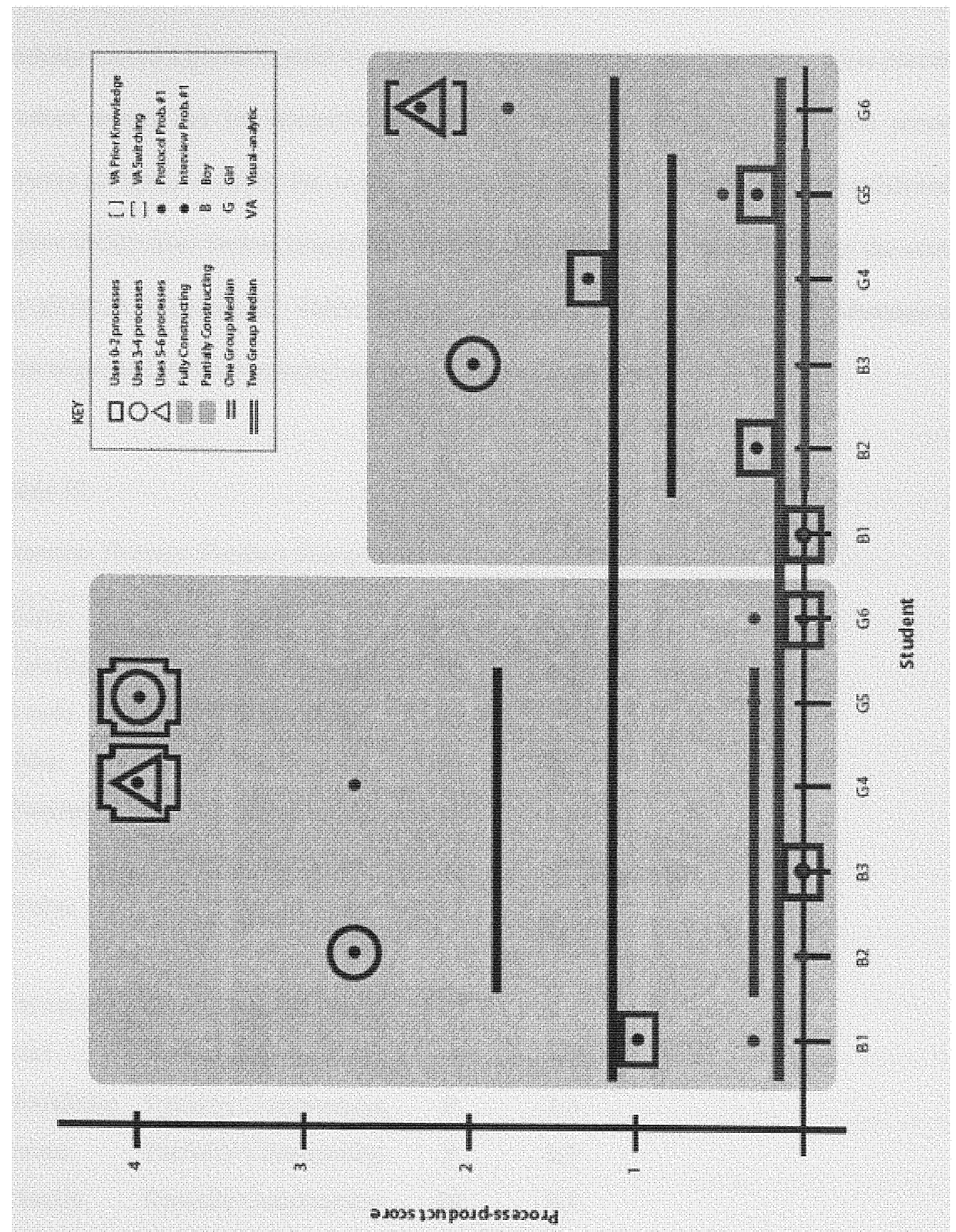

Figure 5. Process-product score and mental processes used by students in problem \#1. 
with written text or numerically. Actually, only two females of the total of twelve students scored a product point by providing the correct answer. Therefore, these processproduct scores can be interpreted as process scores only, wherein the term process denotes a student's ability for visual-analytic processing. In general, females exhibited a higher ability than males. Examples showing how a rubric was used to assign the scores are given in Appendix D. Table 10 shows the scores for all students (pseudonyms are used to protect their anonymity) separated by gender and group.

Table 10

Interview Process-Product Scores

\begin{tabular}{llcc}
\hline Name & \multicolumn{1}{c}{ Group } & Gender & Score \\
\hline Camille & Fully Constructing & F & 4.0 \\
Ronda & Fully Constructing & F & 4.0 \\
Omar & Fully Constructing & M & 2.7 \\
Damian & Fully Constructing & M & 1.0 \\
Lilly & Fully Constructing & F & 0.0 \\
Walter & Fully Constructing & M & 0.0 \\
& & & \\
Stevondra & Partially Constructing & F & 2.3 \\
Yuri & Partially Constructing & M & 2.0 \\
Madeleine & Partially Constructing & F & 1.3 \\
Salma & Partially Constructing & F & 0.3 \\
Charlie & Partially Constructing & M & 0.3 \\
Albert & Partially Constructing & M & 0.0 \\
\hline
\end{tabular}


For the specific score of manipulating a mental image, the results neither showed a difference between experimental groups nor between females and males. Several mental image manipulations that students made while solving the interview problem were: visualizing that there are two square cross-sections in the geometric structure of an octahedron, combining and separating two square pyramids that share their bases, and visualizing right triangles on which to apply the Pythagorean theorem. Of these, the most significant is the first. Because of this mental manipulation, of the two students who obtained four points in the process-product score, one expressed surprise when realizing the equivalence between the length of the diagonal of the square and the height of the octahedron. There is a clear difference, however, in terms of group differences when looking at the total scores. The median of 1.85 for the group fully constructing models was more than twice the median of 0.80 for the second experimental group partially constructing models with GSP. Similarly, the median of 1.80 for females was more than twice the median of 0.65 for males.

The second aspect of the information analyzed in the interviews was students' recollection of both visual and analytic prior knowledge. Figure 5 depicts that only three of the twelve students made both visual and analytic references to prior knowledge. In fact, these three students were females, and obtained three of the highest scores. The visual features they recalled were: the $\sqrt{ } 2$ to 1 ratio of the length of the hypotenuse of a right isosceles triangle to its legs' length; the altitude of a regular square pyramid as defined by the segment connecting the pyramid's top vertex to the center of the square base; and the geometric structure of an octagon. A student referred to this last feature as: "an octahedron is four triangles connected facing up and four triangles facing down." As 
for analytic reasoning, most of the students recalled the Pythagorean theorem as a relation that could lead them towards the solution of the problem.

The third aspect was the ability to switch between visual and analytic reasoning during the solution of the problem. The data showed once again that only females exhibited the ability to switch back and forth more than one time. Figure 5 shows that these two females obtained the highest scores. During the interview, for example, Ronda explained her reasoning while switching between visual (V) and analytic (A) reasoning: -Every side of the octagon equals one (A).

-I can use the square (V).

-I can use its diagonal, which measures $\sqrt{ } 2(A)$.

-So the height of the octahedron is $\sqrt{2}(\mathrm{~V})$.

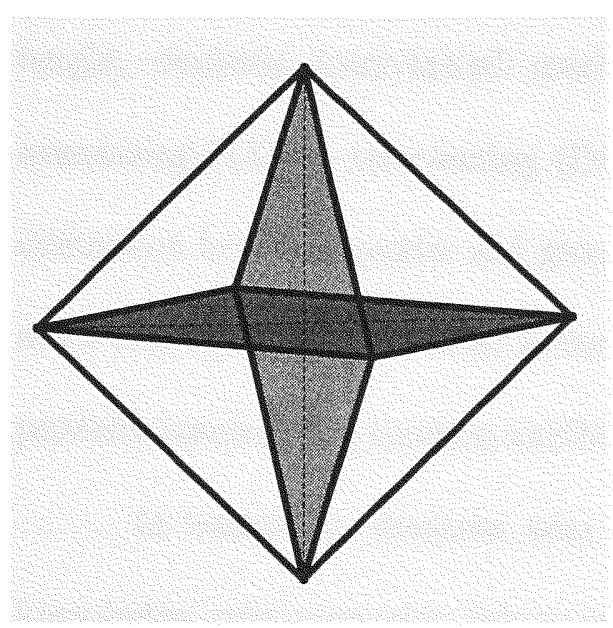

Figure 6. Diagonals of horizontal and vertical cross-sections.

Ronda switches from analytic to visual reasoning, and vice versa. She first recalls a given property of the octahedron that refers to its parts, namely the length of the edges. Ronda 
proceeds to visualize the horizontal square cross-section, and hopes to find a meaningful relationship. With an almost immediate reaction, she recalls the analytic encapsulation of the relation between the length of the legs and the hypotenuse of an isosceles triangle. During the treatment of the study this relation was studied when exploring properties of a cube's diagonal. Finally, as shown in Figure 6 Ronda makes the most meaningful relation by visualizing a $90^{\circ}$ spatial rotation of the octahedron. This transformation consists of rotating the horizontal square cross-section into a position in which the cross-section ends up vertically. As indicated by the parentheses, this sequence of switching from analytic to visual reasoning, and vice versa, was coded as AVAV in this study.

The fourth and last aspect was the number of whole-part processes used by the students. Again, Figure 5 shows that students using a greater number of these processes obtained a higher process-product score. For the problem of finding the octahedron's height, students interviewed used rotation (ROT), reflection (REF), slicing (SLI), connecting (CON), intersecting (INT), and combining \& separating $(\mathrm{C} \& S)$. As for the distinction between gender and group, once again this data showed evidence that females more than males, and students in the group fully constructing models more than the group partially constructing models, exhibited a higher frequency for using these processes.

In summary, students who were able to recall both analytic and visual prior knowledge, who were also able to switch between analytic and visual reasoning, and who used more than three whole-part processes, resulted in having a higher visual-analytic ability as measured by the process-product score. 
Protocol problems \# 1 through \# 4 were administered to students after the third and sixth weeks of the treatment. In particular, problems \# 1 and \# 2 were administered to students individually prior to the interviews on problem \#1. The students spent ten minutes working individually on each of these problems. The purpose was to trace the development of students' visual-analytic processing abilities based on the processproduct score and the frequency with which students used the nine whole-part processes. Problems \#1 and \#2 were administered after the third week, whereas problems \#3 and \#4 were administered after the sixth week.

Aspects analyzed in protocol problems. Two aspects were analyzed in the data collected for the four protocol problems. The first aspect analyzed was the processproduct score. This score differs from the one used for the interviews in that the mental image component is replaced with a sketch component. During the interviews the researcher specifically looked at how students manipulated their mental images. For the individual work collected in the protocol problems, the process score had three components: ability to recall, to sketch a visualization of the problem, and to explain a solution by writing or numerically. Table 11 shows that the mean process-product scores correspond with van Hiele levels, a comparison that gives validity to the scores.

The median score for the 48 data points was 0.5 , with $77 \%$ of the scores between a score of 0.0 and 1.0. Only three scores were in the range 3.1 to 4.0 , all of them achieved by Camille. As it will be evident in all four sources of data, Camille excelled in this study. A detailed account on her reasoning processes will be provided in the next section to serve as a model for other females. Again, as in the interview data, this source of data 
showed evidence that females more than males, and students in the group fully constructing models more than the group partially constructing models, exhibited a higher frequency of visual-analytic processing, as measured by the process-product score.

Table 11

Individual Process-Product Scores and van Hiele Level

\begin{tabular}{lcccccccc}
\hline Name & Group & Gender & P\#1 & P\#2 & P\#3 & P\#4 & Score & $\begin{array}{c}\text { Van Hiele } \\
\text { Level }\end{array}$ \\
\hline Damian & E1 & M & 0.3 & 0.8 & 2.5 & 0.5 & 1.03 & 2 \\
Omar & E1 & M & 0.0 & 0.8 & 2.5 & 0.8 & 1.03 & 2 \\
Walter & E1 & M & 0.0 & 0.0 & 1.8 & 0.5 & 0.58 & 1 \\
Camille & E1 & F & 3.0 & 4.0 & 4.0 & 4.0 & 3.68 & 3 \\
Ronda & E1 & F & 0.3 & 1.3 & 2.5 & 0.5 & 1.15 & 2 \\
Lilly & E1 & F & 0.3 & 0.0 & 0.0 & 0.5 & 0.20 & 1 \\
& & & & & & & & \\
Albert & E2 & M & 0.0 & 0.0 & 0.5 & 0.5 & 0.25 & 1 \\
Charlie & E2 & M & 0.0 & 1.0 & 0.0 & 0.5 & 0.38 & 1 \\
Yuri & E2 & M & 0.0 & 0.3 & 0.5 & 0.0 & 0.20 & 1 \\
Madeleine & E2 & F & 0.0 & 0.0 & 0.8 & 0.5 & 0.33 & 1 \\
Salma & E2 & F & 0.5 & 0.0 & 2.5 & 0.5 & 0.88 & 1 \\
Stevondra & E2 & F & 1.8 & 0.8 & 0.8 & 2.2 & 1.40 & 2 \\
\hline
\end{tabular}

The second aspect analyzed was the number of whole-part processes used by the students while solving the problems. Table 12 lists instances in which at least one student used each of the processes. The nine processes tabulated are combining \& separating (C\&S), translating (TRA), dilating (DIL), rotating (ROT), reflecting (REF), slicing (SLI), folding (FOL), connecting (CON), and intersecting (INT). For problem \#1 (finding the octahedron's height), the data revealed that six whole-part processes were used by at least one student. Both for problem \#2 (sketching a solid represented by a given algebraic 
expression of two variables), and problem \#3 (finding an algebraic expression of two variables given the name of the solid), the data revealed that only three processes

Table 12

Usage of Whole-Part Processes in Protocol and GSP Problems

\begin{tabular}{lccccccccc}
\hline Problem & C\&S & TRA & DIL & ROT & REF & SLI & FOL & CON & INT \\
\hline P\#1 & $\checkmark$ & & & $\checkmark$ & $\checkmark$ & $\checkmark$ & & $\checkmark$ & $\checkmark$ \\
P\# 2 & & $\checkmark$ & & & & & $\checkmark$ & $\checkmark$ & \\
P\#3 & & $\checkmark$ & $\checkmark$ & $\checkmark$ & & & & & \\
P\# 4 & & $\checkmark$ & & $\checkmark$ & & $\checkmark$ & & $\checkmark$ & \\
& & & & & & & & & \\
GSP\#5 & $\checkmark$ & & $\checkmark$ & $\checkmark$ & & & & $\checkmark$ & $\checkmark$ \\
GSP\#6 & $\checkmark$ & & $\checkmark$ & $\checkmark$ & & $\checkmark$ & & $\checkmark$ & \\
GSP\#7 & $\checkmark$ & $\checkmark$ & & $\checkmark$ & & $\checkmark$ & & $\checkmark$ & \\
GSP\#10 & $\checkmark$ & & $\checkmark$ & $\checkmark$ & & & & $\checkmark$ & $\checkmark$ \\
\hline
\end{tabular}

were used by at least one student. These two problems were more abstract, therefore more difficult, than the other two. In problem \#4 (finding the ratio of the area of a square cross-section of the dual to the area of a face of a cube), the number of these processes used by at least one student was four.

Visual-analytic development as measured by the process-product score. The process-product scores revealed that students' development of visual-analytic abilities between the third and sixth weeks was minimal. However, as shown in Appendix F, the data revealed that specific development did occur when analyzing the sketching process. The process-product scores were categorized into three types as follows: 
1. Scores which included at least a component score of 1.0 ,

2. Scores which included a component score of 0.5 or 0.3 , and

3. Scores whose component scores were all 0.0 .

The data from this source showed that the percentage of students in the third type, namely those getting a 0.0 on all components, drastically reduced from $23 \%$ to $6 \%$ after 3 weeks. Similarly, the percentage of students exhibiting the second type, namely getting a 0.5 or 0.3 on the sketch and solution components of the score, increased from $17 \%$ to $27 \%$ after the 3 weeks.

According to the pre-established rubric for the process-product score (see Appendix D), the two lower levels for the sketching process were evaluated as follows:

1. A score of 0.0 corresponded to: not displaying a sketch, displaying a twodimensional sketch, or displaying a weak or irrelevant three-dimensional sketch.

2. A score of 0.5 corresponded to: sketching a relevant three-dimensional object and partially using it in the solution, or sketching a two-dimensional object which coded three-dimensional information.

Improvement in sketching was evidenced as shown by students moving from the 0.0 category to the 0.5 category after 3 weeks (see Appendix F). In general, this analysis showed that males and students in the group partially constructing models with GSP moved from displaying weak sketches to drawing relevant sketches.

Characterization of protocol problems. The use of visual or analytic reasoning, working a problem backwards, abstracting an equivalent class of objects, and using transitivity are four strategies that characterize the four protocol problems. One student's solution to all problems is used as an example for each of the four characterizations. 
The analysis of problem \#1, a problem which can be characterized as suitable for the use of either visual or analytic reasoning, resulted in meaningful findings for one girl's strategy and for one other strategy used by three students. The girl, Camille, did not achieve a perfect score because she forgot to square the term $\sqrt{2} / 2$ when applying the Pythagorean theorem. In addition to setting up the problem correctly, the strategy she used is important. Camille visualized the octahedron as the combination of two square pyramids. She decided to make computations based on the top pyramid. Her plan was to find the pyramid's height and then multiply it by two to obtain the height of the octahedron. Her solution included visualizing a right triangle whose hypotenuse was 1 unit, and whose legs were the height of the pyramid and half the diagonal of the pyramid's square base. This student used an analytic encapsulation for computing the length of half the diagonal. She labeled it $\sqrt{2} / 2$ without providing any computations. The strategy used by three students was inappropriate for a $10^{\text {th }}$ grade level. As in other problems of this study, students used visual estimates of lengths based on visual proportioning. One of the three students responded, "The height of the octahedron is twice as long as the sides," without justification. As presented in the previous section, Ronda's solution to the same problem starkly contrasts with the strategy shared by these three students.

The analysis of strategies to solve problem \#2 showed that one student used knowledge transfer and the ability to work the problem backwards as explained by Polya (1957). The possibility of working backwards surfaces after a student has provided algebraic expressions describing the geometric structure of several solids. Problem \#2 asked for the solid given the expression. The strategies analyzed provide a clear example 
of how analytic reasoning supports visual reasoning. The coefficients of the analytic expression represent the number of faces, a visual feature of the solid. Additionally, the two variables in the expression elicit the fact that the surface area consists of two different shapes. Only two females were able to transfer prior information to the backwards situation. Two males correctly realized they needed six polygons but sketched a cube in response to the question. Their answer violated the given information that not all areas were equal. Two females, one of them being Camille, correctly proceeded to sketch a rectangular prism with two squares and four rectangles. One other girl used a
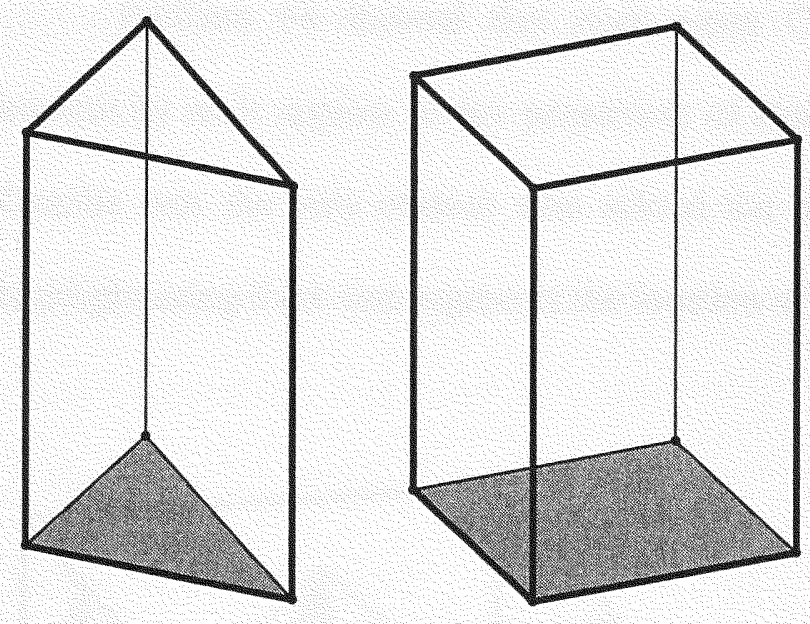

Figure 7. Two prisms representing $3 \mathrm{x}+2 \mathrm{y}$ and $4 \mathrm{x}+2 \mathrm{y}$.

square and a triangle and obviously failed in her attempt to sketch the solid. This girl's processing of information showed lack of flexibility. She insisted on different attempts to use the triangle and the square. But more important, is pointing out that in a constructivist setting the teacher should take advantage of the situation by changing the expression 
from $4 x+2 y$ to $3 x+2 y$. Hopefully, the student would sketch a rectangular prism with triangular base, as shown in Figure 7.

Problem \#3 showed that Camille recognized the concept of equivalent class. Of the twelve students, only this student realized that the same algebraic expressions could be used for two similar solids even though they are of different size. Four other students gave a correct expression for only one of the two objects. A common mistake evidenced by the students' answers was that the number of faces was not represented by the coefficient but by the exponent. For example, one student's algebraic expression to represent the surface area of the rectangular prism was $x^{4}+2 y$.

Problem \#4 showed that once again Camille correctly used the process of transitivity as it applies to the projection of elements of a solid onto different planes. Camille was the only student who solved the problem correctly. Her strategy was to highlight using thick line segments the location of the horizontal square cross-section of

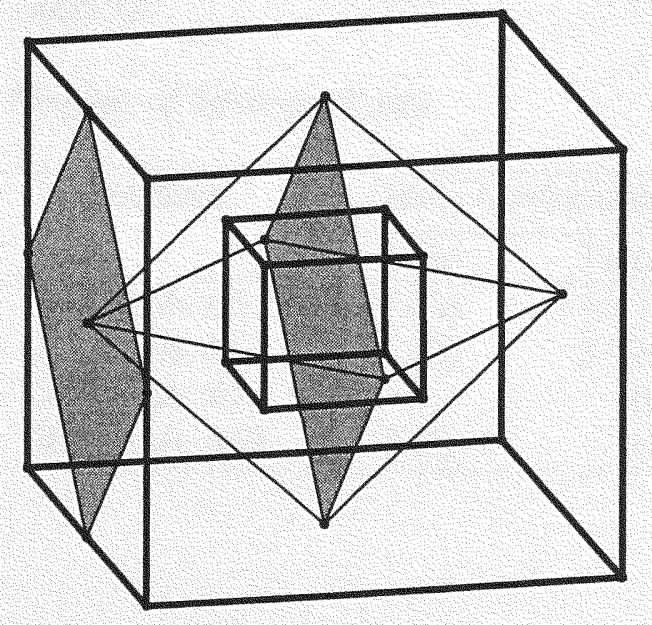

Figure 8. Vertical square cross-section projected to lateral face of larger cube. 
cross-section to one of the faces of the cube, as shown in Figure 8. The projection utilized was an implicit use of transitivity and allowed the student to conclude that the horizontal square cross-section is congruent to the vertical square inscribed on a lateral face of the cube. This student's solution to the problem shows that students are capable of using multi-step visualizations.

\section{GSP Problems}

Aspects analyzed in GSP problems. Four aspects were analyzed in this source of data. The first aspect analyzed was the type of strategy used to solve mathematical problems tied to the constructions which students created in GSP. Both experimental groups used a similar number of strategy types. However, males used less than half the number of strategy types used by females. As presented in Table 13, the data indicated that females may be more versatile than males in devising different strategies for the same problem.

Table 13

Number of Strategy Types

\begin{tabular}{lcccccc}
\hline \multirow{2}{*}{ Problem } & \multicolumn{2}{c}{ Fully Constructing } & & \multicolumn{2}{c}{ Partially Constructing } \\
\cline { 2 - 3 } \cline { 5 - 6 } & Male & Female & & Male & Female \\
\hline GSP Prob. \#5 & $1(\mathrm{~A})$ & $3(\mathrm{~A}, \mathrm{~B}, \mathrm{C})$ & & $1(\mathrm{~A})$ & $2(\mathrm{~A}, \mathrm{C})$ \\
GSP Prob. \#6 & $1(\mathrm{D})$ & $2(\mathrm{D}, \mathrm{E})$ & & $1(\mathrm{~F})$ & $3(\mathrm{D}, \mathrm{F}, \mathrm{G})$ \\
GSP Prob. \#7 & 0 & 0 & & 0 & $1(\mathrm{H})$ \\
GSP Prob. \#10 & $1(\mathrm{I})$ & $1(\mathrm{I})$ & & $1(\mathrm{I})$ & $2(\mathrm{I}, \mathrm{J})$ \\
\hline
\end{tabular}

The second aspect analyzed was whether students worked individually or in groups. Calculated using the data shown in Appendix I, students' preferred working in 
groups in $76 \%$ of the occasions. Of the 48 total possible occasions, with each of 12 students solving 4 problems, 45 data points were available for the analysis. Three students were absent the day they were assigned to work on problem \#7. Table 14 shows the number of strategy types in which students worked individually. Seven types of strategies (B, C, D, E, G, H, and J) were used by females working individually. The fact that no males chose to work individually implies that there is a cause for the interpretation made from the data of Table 13. Because females chose to work individually, they generated more strategies than males. Finally, there were no clear differences between the two experimental groups which can be derived from the data on Table 14.

Table 14

Number of Strategy Types in which Students Worked Individually

\begin{tabular}{lcccccc}
\hline \multirow{2}{*}{ Problem } & \multicolumn{2}{c}{ Fully Constructing } & & \multicolumn{2}{c}{ Partially Constructing } \\
\cline { 2 - 3 } \cline { 5 - 6 } & Male & Female & & Male & Female \\
\hline GSP Prob. \#5 & 0 & 2(B, C) & & 0 & $1(\mathrm{C})$ \\
GSP Prob. \#6 & 0 & 1(E) & & 0 & $2(\mathrm{D}, \mathrm{G})$ \\
GSP Prob. \#7 & 0 & 0 & & 0 & $1(\mathrm{H})$ \\
GSP Prob. \#10 & 0 & 0 & & 0 & $1(\mathrm{~J})$ \\
\hline
\end{tabular}

The third aspect analyzed was the success in explaining the answers to the problems tied to the GSP constructions. A coding system was developed using 1 for the students who were successful and 0 for those not successful. As shown in Table 15, the students completed $76 \%$ of the constructions. In terms of completing the GSP constructions, both experimental groups and genders completed about the same number 
of them. However, only $16 \%$ of the questions were answered correctly, with females and students from the group fully constructing models outperformed males and those in the other experimental group. An important observation is that the relatively high percentage (76\%) for completing the constructions of the solids does not guarantee mathematical understanding of the solids' properties and relations, as reflected by the low percentage $(16 \%)$ of correct answers. Even though students' spatial visualization abilities may have developed after making GSP constructions, mathematical understanding remained low. Mathematical understanding was also low as evidenced in the process-product scores for the protocol problems.

Table 15

Number of Complete GSP Constructions (C) and Correct Solutions (S)

\begin{tabular}{|c|c|c|c|c|c|c|c|c|}
\hline \multirow[t]{3}{*}{ Problem } & \multicolumn{4}{|c|}{ Fully Constructing } & \multicolumn{4}{|c|}{ Partially Constructing } \\
\hline & \multicolumn{2}{|c|}{ Male } & \multicolumn{2}{|c|}{ Female } & \multicolumn{2}{|c|}{ Male } & \multicolumn{2}{|c|}{ Female } \\
\hline & $\mathrm{C}$ & $\mathrm{S}$ & $\mathrm{C}$ & $\mathrm{S}$ & $\mathrm{C}$ & $S$ & $\mathrm{C}$ & $\mathrm{S}$ \\
\hline GSP Prob. \#5 & 3 & 0 & 3 & 2 & 2 & 0 & 3 & 1 \\
\hline GSP Prob. \#6 & 3 & 0 & 3 & 0 & 2 & 2 & 3 & 2 \\
\hline GSP Prob. \#7 & 0 & 0 & 0 & 0 & 0 & 0 & 1 & 0 \\
\hline GSP Prob. $\# 10$ & 3 & 0 & 3 & 0 & 2 & 0 & 3 & 0 \\
\hline
\end{tabular}

The fourth and last aspect analyzed was the number of whole-part processes used by the students. Table 12 showed that GSP problems elicited the use of more processes than the protocol problems. This was partly due to the dynamic nature of GSP, which allows students to use the rotation process in all GSP problems. It is also due to the construction process which obviously involves connecting vertices after finding their 
location, and requires the intersection of mathematical objects. Moreover, all the GSP problems are characterized by the use of the combining and separating $(\mathrm{C} \& S)$ process. Neither reflecting (REF) nor folding (FOL) was possible with the current version of GSP. Moreover, the three-dimensional technique utilized for the construction of the models was not sufficiently versatile as to permit the reflection of solids or the folding of parts of solids.

Characterization of GSP problems. Each of the four GSP problems provides insight into how students use visual-analytic processing strategies. In problem \#5 most of the students devised an incorrect procedure in an attempt to locate the centroid of the triangles of the octahedron. This same mistake was reported by July (2001). The students located the midpoint of each side of the triangle first. They then connected one midpoint to the opposite vertex, correctly constructing a median. But on their next step, they connected the other two midpoints. The intersection of this segment with the median was incorrectly labeled as the centroid. The resulting dual was not a cube but a rectangular prism with four rectangles on the lateral faces and two squares on the top and bottom faces. One of the benefits of using GSP referred to in the literature is that it provides feedback visually (Dreyfus, 1990). However, this example shows that even though the students were not seeing a cube, they continued working on their incorrect construction, as shown in Figure 9. When students are excessively focusing on a procedure, care must be provided by the teacher to ensure that the students make meaningful internalizations of the mathematical definitions and concepts. 


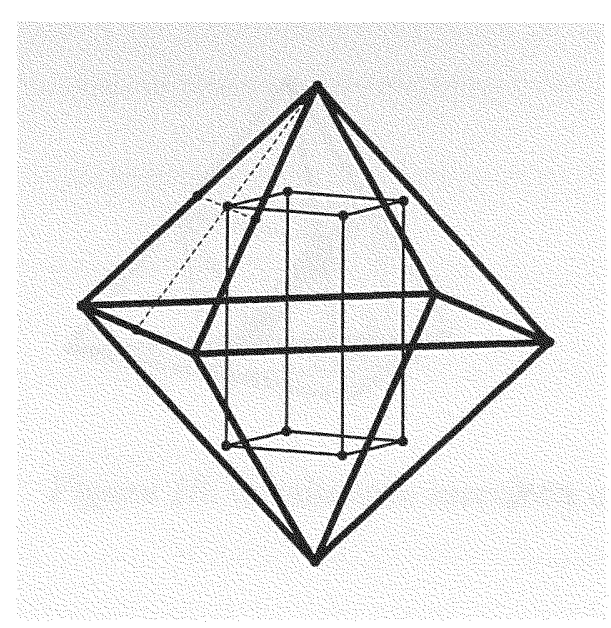

Figure 9. Incorrect centroid location.

Although the purpose of problem \#6 was to present a non-example of the concept of duals, six students called the inscribed octahedron "the dual of the tetrahedron." However, this problem provided two interesting observations. First, while observing a student rotate his GSP construction, the researcher noted an interesting problem with the construction's perspective. The student had stopped the rotation such that the tetrahedron looked like a square with two diagonals. The researcher asked the student if the diagonals were perpendicular. The student replied negatively incorrectly justifying his answer: "the construction does not look like a perfect square." Actually, the two diagonals are perpendicular, and polyhedra can be used to study perpendicularity of lines in space, especially when they don't intersect.

The second observation is that one of two questions in problem \#6 was the question most students answered correctly. Although students did not justify their visual 


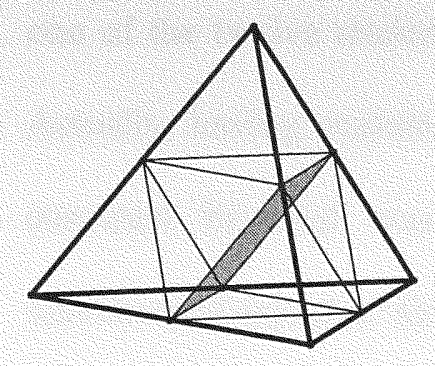

Figure 10. Octahedron inscribed in tetrahedron.

estimates of the 2 to 1 ratio (length of the smaller tetrahedron's edge to length of the larger tetrahedron's edge), most of them were able to visualize the octahedron inscribed in the tetrahedron, as shown in Figure 10. Most students correctly reported that the object constructed by connecting all of the midpoints of a tetrahedron's edges was an octahedron. GSP's hide/unhide tool permitted them to uncover the inscribed object after hiding different portions of segments previously constructed. As the students hid some of these objects, they rotated the tetrahedron from a control button in order to facilitate the selection of other objects they wanted to hide. The rotation capability of GSP had an effect in allowing students to recognize the inscribed object.

The idea of using another object to facilitate students' visualization of a particular object surfaced in observing students construct the icosahedron in problem \#7. For example, identifying and describing the geometric structure of an anti-prism supports the visualization of the icosahedron. Actually, the process of combining and separating (C\&S) can be applied to visualize the icosahedron. Once students understood how to construct the anti-prism, they were able to add a pentagonal pyramid to both pentagons of the anti-prism, thus forming the icosahedron by the combination of three objects. Only 
one of the twelve students was able to complete the construction of the dodecahedron. Actually, having students find 20 centroids on each of the faces of the icosahedron to construct its dual was excessively procedural and not practical. Students were unmotivated and thus did not complete this construction, as shown in the next to the last row of Table 15.

Finally, problem \#10 permitted the researcher to observe how students worked with the concept of infinity. In the dialogue that follows, $\mathrm{T}$ stands for the teacher and $\mathrm{S}$ stands for the student. A student was asked the question:

T-What is the dual of the dual of the dual of a tetrahedron?

S-A tetrahedron.

T-Why is that so?

S-Because the dual of a tetrahedron is always a tetrahedron.

T-And if I ask for the dual of the dual of the dual...infinitely many times...

S-It's still a tetrahedron.

T-Can you describe what that tetrahedron looks like?

S-It's a point.

In reference to the dual of a cube, a student was asked:

T-What is the dual of the dual of a cube?

S-The dual of the dual?

T-Yes, you know what the dual of a cube is, right?

S-Yes.

T-And what is the dual of that solid?

S-A cube. 
T-Good. So, what is the dual of the dual of the dual of the cube? Yes three times!

S-It's an octahedron.

T-And if I say dual of the dual five times? Or seven? Or nine times?

S-For any odd number, it is still an octahedron.

T-And for an even number of times?

S-It's a cube. That's neat!

These are clear examples of students' visual-analytic processing strategies with the added benefit of using mathematical abstractions which do not require numerical computations. Elements of advanced mathematics, for example the concept of limit, are evident in these teacher-student dialogues.

Field Notes

Field notes were taken every day during the 6-week treatment. The purpose of the field notes was to capture details of students' visual processing strategies as they solved classroom problems. If further detail was necessary, video tapes were available to complement the notes. The students in the experimental groups used GSP $50 \%$ of the time to solve three-dimensional tasks. On the days in which GSP was not used, manipulatives were made accessible to the students. However, on the days in which GSP was used, manipulatives were available if students requested them. Different groups of four students were assigned by the teacher every day. The students were encouraged to construct three-dimensional solids as they solved the problems of the treatment. By the end of the study, 28 field note entries of students working on the problems were recorded.

Two aspects were analyzed in this source of data. The first aspect analyzed was whether students were successful $(1)$ or not $(0)$ in solving the problems, as shown in 
Table 16. The researcher informally interviewed individual students as they sat in their groups solving the problems. A record was kept of the student's success in explaining the solution to the problem chosen (see Appendix J). The analysis indicated that 11 of 13 students who used three or more whole-part processes were successful in their explanation of the solution to the problem. This is a significant observation because the 11 observations correspond to $39 \%$ of the 28 entries recorded. Additionally, whether or not using a high number of whole-part processes, 9 females and 11 males were successful in explaining their solutions, representing $71 \%$ of the records. In terms of success or using three or more processes per problem, there were no differences between the experimental groups.

The second aspect analyzed was the number of whole-part processes used by the students. Table 16 shows that all processes were used by both males and females. When considering problems only after the third week, seven females used 15 processes for an average of 2.1 processes per girl, and eight males used 19 processes for an average of 2.4 processes per boy. The processes of combining and separating (C\&S), dilating (DIL), rotating (ROT), and connecting (CON) were the most frequently used. Fourteen of the 28 problems were solved in the last 3 weeks of the treatment, the period in which the differentiation was made between the two experimental groups. During this time, students in the group fully constructing the models used 22 processes and the other group used only 14. These frequencies correspond to 2.4 processes used per student in the first group and 2.3 processes per student in the second group. Therefore, there is neither a difference among groups nor among gender. As evidenced in the interviews, the data 
from the field notes showed that students who used three or more whole-part processes were more likely to be successful in explaining a problem's solution.

Table 16

Classroom Usage of Processes and Success Score

\begin{tabular}{cccccccccccc}
\hline Gender & Score & Group & C\&S & TRA & DIL & ROT & REF & SLI & FOL & CON & INT \\
\hline F & 0 & E1 & & & $\checkmark$ & & & & & $\checkmark$ & \\
M & 0 & E2 & $\checkmark$ & & & & & & $\checkmark$ & $\checkmark$ & \\
F & 0 & E1 & & & & & & & $\checkmark$ & & \\
M & 1 & E1,E2 & & & & & & & & $\checkmark$ & $\checkmark$ \\
M & 0 & E1 & & $\checkmark$ & & & & & & & \\
F & 1 & E1 & $\checkmark$ & & & $\checkmark$ & & & $\checkmark$ & \\
F & 1 & E2 & $\checkmark$ & & & & $\checkmark$ & & & $\checkmark$ & \\
M,F & 1 & E2 & $\checkmark$ & & $\checkmark$ & & & & $\checkmark$ & $\checkmark$ & \\
M & 1 & E2 & & & $\checkmark$ & & & & & & \\
F & 1 & E1 & & & & & & & & $\checkmark$ & \\
M & 1 & E1 & $\checkmark$ & $\checkmark$ & $\checkmark$ & $\checkmark$ & & $\checkmark$ & & $\checkmark$ & $\checkmark$ \\
F & 1 & E1 & $\checkmark$ & $\checkmark$ & & $\checkmark$ & & & $\checkmark$ & $\checkmark$ \\
M & 1 & E2 & & & $\checkmark$ & & & & & \\
M & 0 & E1 & & & & & & & & \\
\hline
\end{tabular}

In addition to the 28 entries on classroom problems, other detailed descriptions of how students use their visual processing strategies follow. In all GSP problems, students translated solids by using the vector tool in GSP. This tool seems to develop students' spatial visualization abilities because it uses an instruction that synthesizes the translation of a solid into the translation of a point. This simplification may encode mental images and reduce cognitive load. For example, in constructing the icosahedron in GSP, students translated a pentagon by an amount described by a vector's length. July (2001) describes how students rotated one of the pentagons 180 degrees 
before connecting the vertices of the top and bottom pentagons, as shown in Figure 11. This translation and rotation was vividly recalled by students when explaining the geometric structure of a pentagonal anti-prism:

S-An anti-prism is made of two pentagons and a belt of triangles between them.

T-What do you mean by belt?

S-The triangles connecting the two pentagons.

T-But why a belt?

S-Remove the two pentagons and flatten the triangles out; they look like a belt.

T-Do the pentagons have the same orientation?

S-No. After translating one with a vector, you have to rotate it 180 degrees.

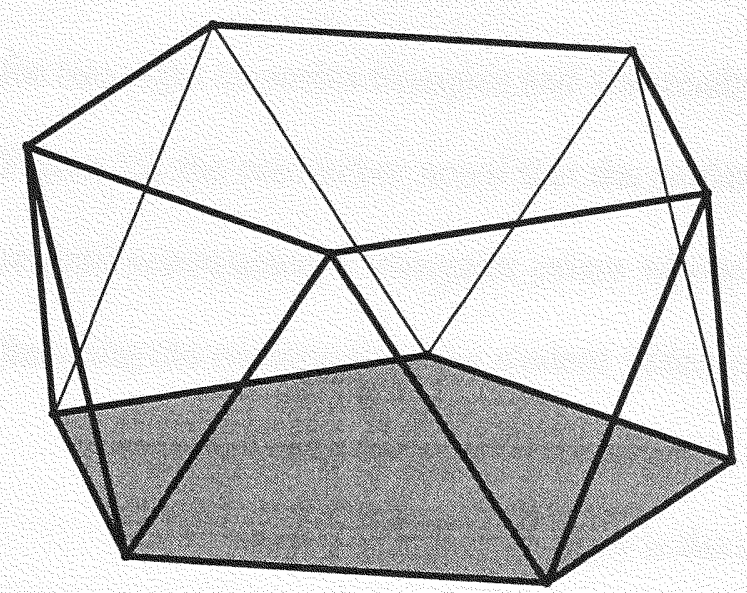

Figure 11. Pentagonal anti-prism.

Another observation helps understand the nature of visualization. Essentially, visualization is a mental process that allows one to see what is not evident in a physical or abstract object or process. A student was asked by the teacher to find the ratio of the 
lengths of the short side to the long side of the Polydron rectangular piece. The teacher gave the following hint: consider slicing a cube through one of its face's diagonal. The student built a cube with six Polydron squares. He then looked in the Polydron class kit

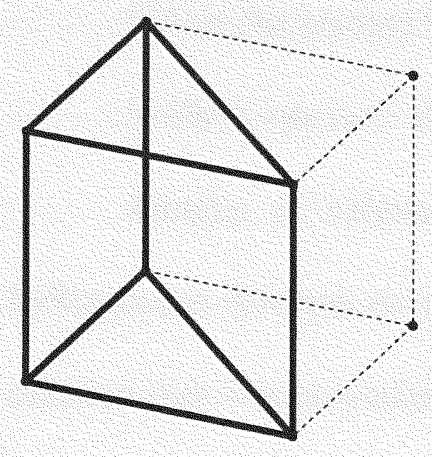

Figure 12. Half the volume of a cube.

for two right isosceles triangles and was ready to make another construction. As shown in Figure 12, the researcher noted that the student was visualizing half the cube. Indeed, the student constructed a triangular prism whose base was a right isosceles triangle. Before finishing the construction, the student yelled out the answer to the original question:

$\mathrm{S}-$ Wow! I can see a rectangle in half a cube.

S-So the length of the rectangle is the square root of two.

T-So, what is the ratio?

S-It's one to the square root of two.

T-And why is it the square root of two?

$\mathrm{S}$-Because it is the same as the diagonal of a square. 
Similarly, in reference to the construction of the dodecahedron, a student asked the teacher why he had chosen such an eye-straining activity for GSP. The student expressed with frustration:

S-I don't know what points to click on.

T-Use the control buttons to zoom in and enlarge your construction.

S-Oh! I can see! Those are centroids.

Finally, in reference to problem \#2 of lesson \#5, most students applied the Pythagorean theorem twice to find the length of segment $\mathrm{AB}$. One student used a visual solution that was powerful for its brevity. The student was asked to explain the solution:

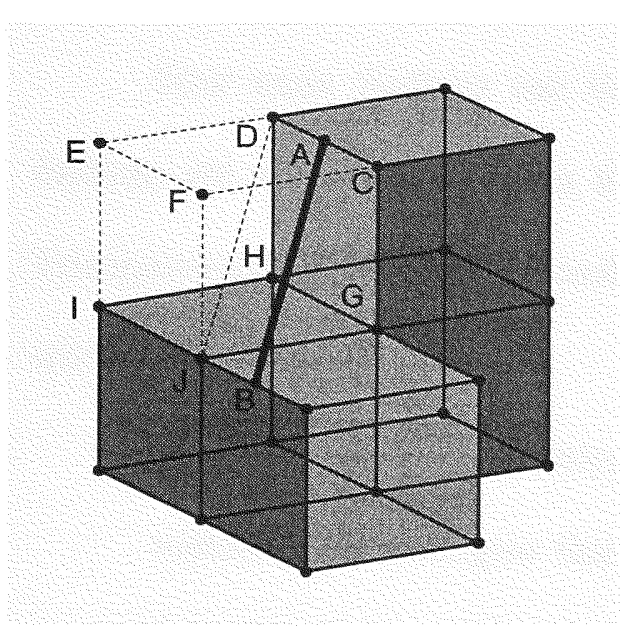

Figure 13. A visual solution

T-How do you know the answer without making computations?

$\mathrm{S}$-The segment is the diagonal of a cube that is not there.

T-Can you explain that?

S-You see, you have to move point A to $\mathrm{D}$, and $\mathrm{B}$ to $\mathrm{J}$. 
T-Please, continue.

S-Then segment JD is the diagonal of a cube that is not there.

T-And how do you know its length.

S-From previous work last week, I know it is the square-root of three.

This student had translated segment $\mathrm{AB}$ to a new position as indicated by the dotted line. The translation obviously preserved the length of the segment. The new position, as shown in Figure 13, coincides with the diagonal of a cube C-J visualized by the student. The highlight of this strategy is that cube C-J resides in the mind of the student. The prototype consists only of four cubes.

\section{Summary of Qualitative Results}

Four main findings can be drawn from the qualitative data. Three of these findings are drawn from within the aspects of the interview data and the GSP data. Triangulation between sources resulted in a fourth finding related to the usage of the nine whole-part processes.

1. Females evidenced during the interview that they were capable of using more sophisticated strategies than males. Only females showed ability in switching from visual to analytic reasoning, or vice versa, as many as three times while solving a problem. Unlike males, females recalled both visual and analytic prior knowledge relevant to the problems posed. Females' use of sophisticated strategies allowed them to obtain a high process-product score.

2. Students in the experimental group fully constructing models, more than the students in the group partially constructing models, showed during the interview that they had a higher visual-analytic ability as measured by the process-product 
score. The students in the first group used a higher number of the nine whole-part processes, as strategies which contributed to the solution of the problem.

3. Data from the GSP source showed that females produced twice as many types of strategies as males because females were more likely to work individually.

4. Considering all four sources of data, the process of connecting (CON), the most elementary of the nine whole-part processes, was the process students correctly used most frequently. From the other eight processes, combining and separating (C\&S) and rotating (ROT) were the next most frequently used as strategies which contributed to the solution of three-dimensional problems. 


\section{CHAPTER V}

\section{DISCUSSION, CONCLUSION, AND RECOMMENDATIONS}

This chapter presents a discussion of the three quantitative and four qualitative results listed in the previous chapter. After encapsulating these results into three general findings, concluding remarks are made about a model proposed for the development of spatial visualization abilities. Finally, this chapter offers recommendations for practice and further research in the area of visualization.

\section{Discussion of the Results}

The first quantitative finding from the ANCOVA on spatial visualization abilities indicated no significant main effect for the group factor. Therefore, connecting visual and analytic reasoning in the experimental groups did not affect students' spatial visualization abilities differently by group. However, an important result related to the effect of the group factor is discussed in the third finding. One reason why there were no significant group differences might have been due to having the same teacher in the three groups. The training the teacher had prior to the study and the experience in using a constructivist setting in the two experimental groups during 6 weeks might have been internalized by the teacher. It is possible that part of the constructivist teaching and learning relationships between teacher and students might have contaminated the control group. Additionally, because a portion of the treatment devoted time to familiarizing students in both experimental groups with GSP, not enough time might have been allocated to differentiate both groups in terms of the differences between fully and partially constructing solids. Furthermore, since the PSVT instrument emphasizes Gestalt processing rather than analytic processing, the test might not be valid for studies such as 
the present one in which students are encouraged to use visual and analytic reasoning. Paired t-tests comparing the improvement between pretest and posttest within the three groups of the study were significant for all groups. Table 3 in chapter 4 showed that there was an improvement of at least 2.5 points between pretest and posttest in all groups. This result shows that the methods of the experimental groups are at least an alternative to the type of teaching and learning used in the control group.

The second quantitative finding from the ANCOVA on spatial visualization abilities indicated a significant main effect for gender, with males performing significantly better than females. This result is in agreement with other research (Ansell \& Doerr, 2000). However, the proportion of variance of spatial visualization ability that is related to gender, excluding group and interaction sources, $\eta^{2}=0.06$, is not high according to Cohen (1988). To obtain more statistical power, each of the six cells in the ANCOVA, varying between 8 and 13 participants for this study, should be increased to at least 30 participants per cell. The 6-week treatment might have not been sufficiently long to allow females to develop their spatial visualization abilities.

The third quantitative finding resulted from analyzing a sub-sample of 33 participants with low pretest scores. An emerging hypothesis as a result of this analysis is that students with low entering spatial visualization abilities may benefit from fully constructing models using GSP. This hypothesis is in agreement with similar research studies in which students with low visualization scores improved more than students who had a high spatial ability (Battista et al., 1982; July, 2001). Additionally, the result brings to light the debate of whether or not students, and what students, should be educated for basic skills, as opposed to for higher thinking processes, or for both. For example, 
according to Delpit (1995), all students can be educated and students lacking basic skills have benefited from just acquiring procedural knowledge. In the study, fully constructing the models resulted in a more procedural approach than partially constructing the models.

The qualitative analysis resulted in four findings. The first two refer to a causeeffect relationship observed during the interviews. The relationship noted is that students who used more mental processes (connecting to prior visual and analytic knowledge, switching between visual and analytic reasoning, and using whole-part processes) obtained a higher process-product score. The relationship was evidenced both for females and for students from the group fully constructing models with GSP. As noted by Sinclair (2004), and unlike students who partially constructed models, these students may have benefited from being aware of the mathematical relationships underlying the constructions they fully constructed using GSP. In addition, the group fully constructing models included two female students who demonstrated the highest ability in using visual-analytic processes (as measured by the process-product score). Examples of the thought processes used by these two females, Camille and Ronda, were presented in chapter 4. Both girls were always on task and constantly participated by asking questions of the teacher and helping other students. It is not clear whether or not being in the group fully constructing models caused an increased interest in the subject by providing the girls with a feeling of ownership resulting from fully constructing the models.

A closer analysis of the components of the product-process score showed that in the interviews each of the components was higher for females than for males and higher for students from the group fully constructing models than from the group partially constructing models. These students' ability to use the processes may have caused a 
higher ability to form mental images. This result is in agreement with Del Grande's (1987) observation that the constructs of image formation and visual perception are two processes necessary to understand three-dimensional geometry.

The third qualitative finding refers to females producing twice as many types of strategies when solving the GSP problems. However, the researcher believes that males produced fewer types of strategies than females not because males were more likely to work in groups but because they were slacking. When working individually, females generated strategies B, C, E, G, H, and J-none of which were used by males.

One of the strategies females used in the construction of duals was to visually check that the distances between the locations of the centroids were equal. While working on this task, one girl asked a critical question: "Why do medians of a triangle intersect at the centroid?" Another strategy utilized by a girl was to use the GSP tool for shading a pentagonal cross-section of an icosahedron and then visualizing other pentagons without the aid of the GSP shading tool. In addition, one girl was able to use multiple steps in deciding that if $\mathrm{x}$ volumes of the dual of a tetrahedron were equivalent to the volume of the original tetrahedron, then $x^{2}$ volumes of the dual of the dual would be equivalent to the volume of the original tetrahedron. Finally, two elementary strategies used by both males and females were to visually estimate ratios and to use GSP's measure tool to calculate ratios. In agreement with Yen (1975), gender differences in spatial visualization ability as measured by the PSVT seemed to result from differences in the way spatial tasks were processed rather than from differences in spatial ability itself.

The fourth qualitative finding was that students correctly used the process of connecting (CON) with a higher frequency than any of the other nine whole-part 
processes. Combining and separating $(\mathrm{C} \& \mathrm{~S})$, and rotating (ROT) were also used correctly with a high frequency. In particular, data from the field notes indicated that males and students in the experimental group fully constructing models used the nine processes more frequently than the other students. Similarly, data from the interviews indicated that females and students in the experimental group fully constructing models used the nine processes more frequently than the other students. However, as presented in Table 17, neither of these two findings had a comparative ratio between 0.0 and 0.5 (a measure which denotes one group's score being at least twice as high as that of the other group).

The two processes of combining and separating (C\&S) and rotating (ROT) may be better understood by students because of the relationships between these processes and other whole-part processes. For example, a composition of reflections across two parallel lines is equivalent to a translation. Similarly, combining and separating has a mathematical relationship with dilation. This relationship is evidenced when exploring a tetrahedron whose edges are dilated by a factor of two. The transformation produces a larger tetrahedron with a volume that is exactly eight times larger than the volume of the original tetrahedron. Since the larger tetrahedron can be separated into eight smaller tetrahedral, the process of combining and separating $(\mathrm{C} \& S)$ is also relevant. Furthermore, the process of combining and separating $(C \& S)$ is also related to reflection as was evidenced in students' solutions of protocol problem \#1. One student's solution, for example, consisted in computing the height of one of the two reflecting pyramids that together form an octahedron.

In summary, as presented in Table 17 , females performed better than males in most of the aspects analyzed. However, the students who fully constructed models with 
GSP did not clearly perform better than those who partially constructed models. Table 17 shows that in most of the aspects analyzed neither of the two groups was dominant. The three labels males, females, and neither appearing in Table 17 were systematically assigned depending on a comparative ratio between the two genders. Similarly, the same systematic procedure was utilized for labeling the dominant experimental group. For example, the comparative group ratio of 0.4 in the first row of Table 17 corresponds to the process-product scores of the interviews. The value was found by using the medians of the two experimental groups extracted from Table 10 in chapter 4 . The median for

Table 17

Findings by Gender and Group

\begin{tabular}{|c|c|c|c|c|c|}
\hline Source & $\begin{array}{c}\text { Aspect } \\
(*)\end{array}$ & $\begin{array}{c}\text { Gender } \\
\text { Ratio } \\
\end{array}$ & $\begin{array}{l}\text { Group } \\
\text { Ratio }\end{array}$ & $\begin{array}{c}\text { Dominant } \\
\text { Gender }\end{array}$ & $\begin{array}{c}\text { Dominant } \\
\text { Group }\end{array}$ \\
\hline \multirow[t]{4}{*}{ Interviews } & PPS & 0.4 & 0.4 & Female & Fully constructing \\
\hline & VAP & 0.0 & 0.0 & Female & Fully constructing \\
\hline & VAS & 0.0 & 0.5 & Female & Fully constructing \\
\hline & WPP & 0.6 & 0.8 & Neither & Neither \\
\hline \multirow[t]{2}{*}{ Protocol Problems } & PPS & 0.3 & 0.4 & Female & Fully constructing \\
\hline & WPP & N/A & N/A & N/A & N/A \\
\hline \multirow[t]{4}{*}{ GSP Problems } & TST & 0.4 & 0.8 & Female & Neither \\
\hline & IND & 0.0 & 0.6 & Female & Neither \\
\hline & SES & 0.4 & 0.4 & Female & Partially constructing \\
\hline & WPP & N/A & N/A & $\mathrm{N} / \mathrm{A}$ & N/A \\
\hline \multirow[t]{2}{*}{ Field Notes } & SES & 1.0 & 1.0 & Neither & Neither \\
\hline & WPP & 0.9 & 1.0 & Neither & Neither \\
\hline
\end{tabular}


the group fully constructing models was 1.85 , whereas for the group partially constructing models the median was 0.80 . By dividing the smaller by the larger number, the comparative ratio of 0.4 denotes that the first group was dominant. From this systematic procedure, it can be deduced that ratios approaching 1.0 imply that the two groups had an equal score, and therefore neither one was dominant. Comparative ratios between 0.0 and 0.5 imply that one group scored at least twice the score of the other group, and therefore the group with the higher score was displayed as dominant.

\section{Conclusion}

The conclusions made in this section are an encapsulation of the seven findings listed in the previous chapter and correspond to each of the research questions:

1. Students' spatial visualization abilities, as measured by the PSVT, were not significantly affected by the treatment. Therefore, connecting visual and analytic reasoning did not affect differently students' spatial visualization abilities while solving three-dimensional tasks across groups.

2. Spatial visualization abilities, as measured by the PSVT, were significantly higher for males than for females in the whole sample of participants. When analyzing the data for a sub-sample of students performing below the $50^{\text {th }}$ percentile in the PSVT pretest, the ANCOVA showed a significant interaction between group and gender. The pairwise analysis showed males benefited significantly from being in the group fully constructing models with GSP in comparison to the group partially constructing the models. The analysis of the sub-sample suggests that students entering the study with low spatial visualization 
abilities may benefit more from fully constructing the models with GSP than from partially constructing the models.

3. Considering all four sources of data, students' whole-part processes such as connecting $(\mathrm{CON})$, rotating (ROT), and combining and separating $(\mathrm{C} \& \mathrm{~S})$ were the most frequently used while solving three-dimensional problems. Students who exhibited a frequent use of these strategies were more likely to recall, sketch, imagine, and explain visualizations related to the problems, as suggested by the process-product score. Although males performed better than females in the quantitative PSVT, females exhibited in the qualitative data a higher frequency for switching from visual to analytic reasoning, and vice versa. Females also more frequently recalled visual and analytic knowledge. Differences between the two experimental groups were not found.

As part of the conclusion, a model for integrating spatial visualization abilities, other mathematical abilities, and visual-analytic processing strategies emerged from the study. As shown in Figure 14, and based on the qualitative findings of this study, the model explains how the integration of three constructs can help students develop strategies for solving three-dimensional mathematical problems. The lower portion of the triangular arrangement of the three constructs indicates that students' spatial visualization abilities correlate with other mathematical abilities as found by others (McGee, 1979). This bidirectional relationship is depicted by the two arrows connecting the two constructs. The node representing the construct of visual-analytic processing strategies connects both to the node of spatial visualization abilities and to the node of mathematical abilities, indicating that a students' utilization of the former construct is 


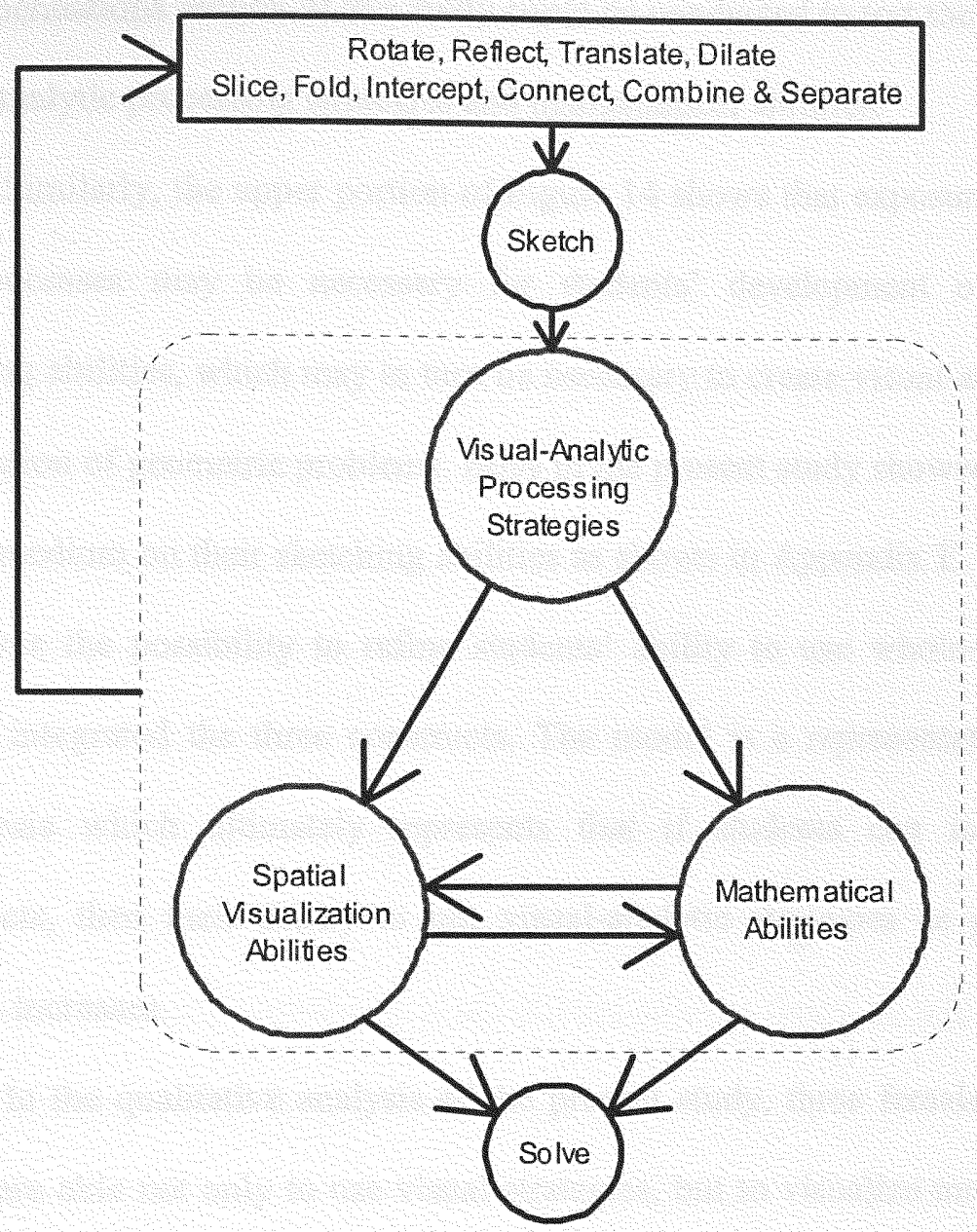

Figure 14. Model for the integration of three constructs.

necessary for the development of the other two. Although the study did not support the link shown in Figure 14 which connects visual-analytic processing strategies to spatial visualization abilities, increasing the duration of the treatment in future studies may support the connection. In fact, the visual-analytic strategies used by students to solve three-dimensional problems during the treatment did not affect the students' spatial visualization abilities differently across groups. Nonetheless, significant improvement was observed from pretest to posttest in each group. Other studies discussed in the 
recommendations section of this study could be conducted to test the connection between visual-analytic processing strategies and mathematical abilities.

Similarly, the upper portion of Figure 14 shows that exposure to the nine wholepart processes may be necessary for students' development of three-dimensional sketching abilities, which may in turn be necessary to create visual-analytic strategies for the solution of geometric problems. Boys in the present study showed improvement from low to medium on their sketching abilities as shown in Appendix F. The returning arrow represents the possibility to refine students' ability to use whole-part processes after having integrated the three constructs. The model is a representation of an emerging hypothesis which ultimately represents that if students can integrate these three constructs, then their ability to use visual-analytic strategies as part of the solution process increases.

In the qualitative analysis of the present study, three females demonstrated that they were able not only to use visual strategies, but to visualize multiple steps required for the solution. These multi-step processes are actually embedded in the questions of the PSVT. However, the quantitative results from analyzing the data obtained from the PSVT demonstrated that males scored significantly higher than females. The apparent discrepancy between the results of the quantitative and qualitative results is resolved by noting that two different constructs are measured in the study: spatial visualization abilities for the quantitative analysis and visual-analytic strategies for the qualitative analysis. Therefore, the results did not need to agree. However, it is important to highlight that the complementarity purpose of the mixed-methods approach was to 
provide through the strategies construct what and how students might be thinking while solving the spatial visualization problems of the PSVT.

\section{Recommendations}

This section presents several pedagogical implications for the teaching and learning of solid geometry and outlines areas for future research and development. Pedagogical Implications

Several pedagogical implications resulting from this study are included in a model for the teaching and learning of solid geometry. The model is shown in Figure 15 and shows how two types of visualization, mathematical and spatial, converge in the study of solid geometry to improve students' spatial visualization abilities. Although the study did not result in significant group differences, and gender differences favoring males persisted, the recommendations made herein address ways in which the experimental error can be reduced in further studies.

The model proposes that students' development of spatial visualization abilities should require four considerations. The first consideration is the use of manipulatives and computer software with the capability to represent three-dimensional models, as were utilized in the present study. A longer time exposure, especially with students who have prior experience with GSP, as was done in the pilot of the present study and as documented by (July, 2001), should result in the desired outcomes. Additionally, a different instructor should teach in the control group.

The second consideration is students' exposure to full-scale models. As suggested by Bishop (1983), with such models, students go through a vicarious experience that may help further develop their spatial visualization abilities. Educational settings taking 


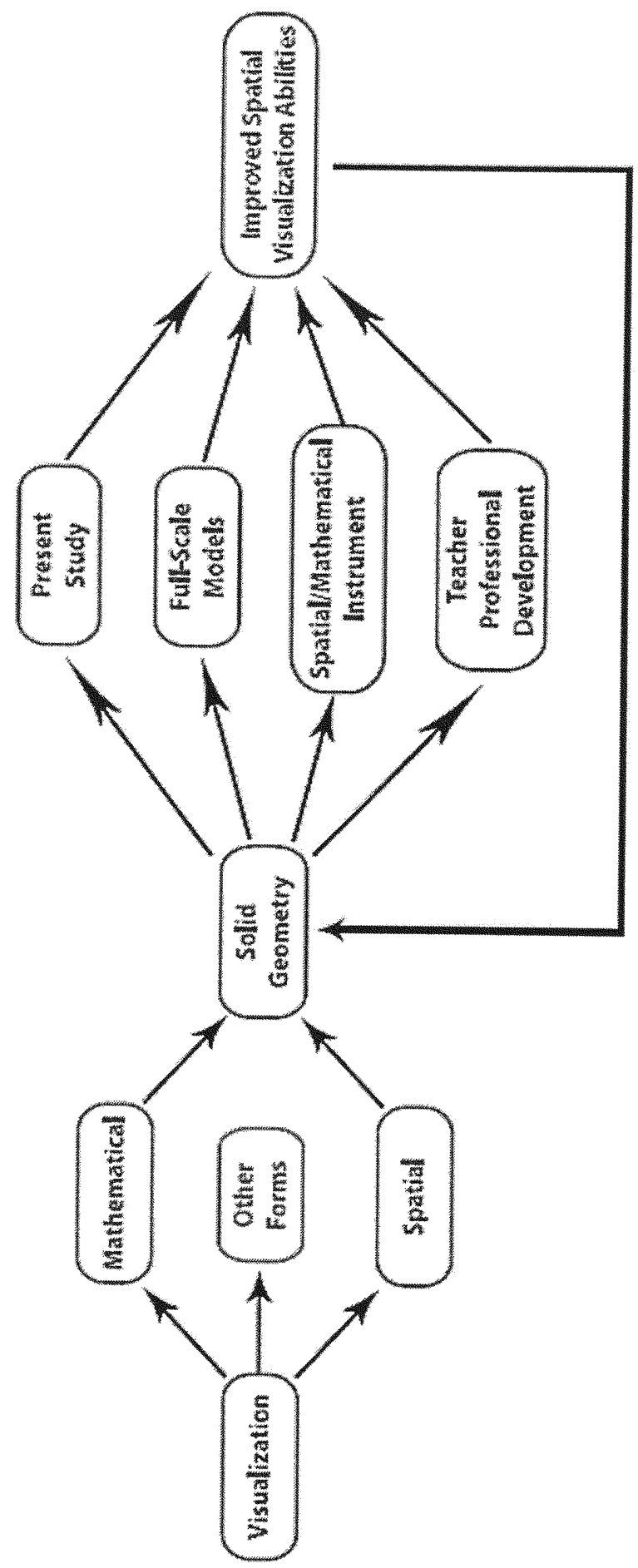

Figure 15. Model for the teaching and learning of solid geometry. 
advantage of the penta-hut could motivate students to engage in three-dimensional geometry tasks such as the ones presented in Appendix B.

Another consideration is the development of three-dimensional spatial ability instruments which require the use of mathematical calculations. These instruments should incorporate mathematical processes such as visual and analytic reasoning to evaluate students' understanding of solid geometry, not only their spatial abilities. Although the literature presents studies in which spatial ability relates to van Hiele levels of geometric thinking, other three-dimensional instruments explicitly integrating mathematical processes should be developed (Del Grande, 1987). Dixon (1997) partly concluded that in her study GSP was not effective in improving students' three-dimensional visualization. She suggested that the non-effect of GSP may have been different if the spatial visualization of students in her study had been measured using a three-dimensional test.

Finally, the need for teachers' attendance to professional development workshops on visualization is another obvious consideration since they are the guides and facilitators of students' learning processes.

As indicated by the arrow connecting back from the box for improved spatial visualization abilities to the box for solid geometry, the process of learning solid geometry should have a dual focus. First, in a geometry class students should use spatial visualization abilities to further develop them. Second, students should develop spatial visualization abilities to use them at least in geometry class.

\section{Future Research and Development}

Several topics which were part of the treatment of the present study need to be expanded and separated for further, more specific investigations. Recommendations are 
made in this section for the following topics: students' transfer of information from one form of representation to another by using analogies; the impact on students' visualization abilities when using full-scale geometric models; the excessive use of either visual or analytic reasoning and how it blocks the mode used less; the development of algebraic notation for writing expressions that describe the geometric structure of solids; and the effect of students' age on the development of spatial visualization abilities.

Representing problems through analogies was part of the treatment of this study. Observing the students working on tasks about analogies permitted the researcher to make a meaningful mathematical conjecture. Considering the analogy between the centroid of an equilateral triangle and the center of mass of a tetrahedron, the researcher conjectures: Is there an analogous point for a hyper-tetrahedron, and where is it located? The sequence for the analogous locations of the points in reference may be $1 / 3,1 / 4,1 / 5$ from the base, corresponding to the second-, third-, and fourth-dimensions. The visual and analytic exploration of this conjecture relates moving from the third- to the fourthdimension. This relation may shed light on specific cognitive processes required for the more basic high school process of moving from the second- to the third-dimension. Furthermore, other ways of using GSP in which students can construct the intersection of volumes as shown in the first problem of lesson \#6 should be developed. The upgrade of current versions of GSP to include the capability of reflecting solids about a plane would be beneficial for students solving these problems.

Research specifically focusing on students' visual-analytic processing as they interact with full-scale models may shed light on how students process this type of information. The penta-hut is a full-scale model developed by the researcher in which one 
vertex of each of four five-foot-side pentagons converge in a saddle-point. Perception of a solid from the inside, walking under and around it, are experiences which may develop students' perceptions of three-dimensional objects. The complementary problems about the penta-hut which are provided in Appendix B may generate significant research questions. Conducting other specific investigations on the use of visual and analytic reasoning is recommended. For example, since students' excessive use of one mode may block the use of the other, this problem could be studied in light of the visual-analytic model to further the understanding of how students coordinate the two modes.

In terms of development, a recommendation is to create a precise and coherent notational system for the algebraic expressions presented in the first lesson of the present study. For these problems, an improved algebraic notation could more clearly represent the features of the geometric structure of solids. Yet another area pertaining to the development of visualization processes which could be investigated is the effect of students' age. The optimal age bracket for the study of visualization has been reported to be between 7 and 12 years of age (Bishop, 1983).

Finally, since mathematics is of a dual visual-analytic nature, the learning and teaching of solid geometry, as well as any other area of mathematics, should include opportunities that enable students to experience both reasoning modes. A deeper mathematical understanding of the properties and relations of the objects studied is achieved when students construct their own knowledge with appropriate visual-analytic scaffolding. Computer software and manipulatives are two educational tools which teachers can use to support the development of students' visual-analytic abilities. 
Alias, M., Black, T. R., \& Gray, D. E. (2002). Effect of instructions on spatial visualization ability in civil engineering students. International Education Journal, 3, 1-12.

Allen, M. J., \& Hogeland, R. (1978). Spatial problem solving as function of sex. Perception and Motor Skills, 47, 348-350.

Amit, M., Fried, M. N. (2002). Research, reform, and times of change. In L. D. English (Ed.), Handbook of international research in mathematics education (pp. 355381). Mahwah, NJ: Lawrence Erlbaum Associates.

Ansell, E. A., \& Doerr, H. M. (2000). NAEP findings regarding gender: Achievement, affect and instructional experiences. In E. A. Silver \& P. A. Kenney (Eds.), Results from the seventh mathematics assessment of National Assessment of Educational Progress (pp. 73-106). Reston, VA: National Council of Teachers of Mathematics.

Arcavi, A. (2003). The role of visual representations in the learning of mathematics. Educational Studies in Mathematics, 52, 215-241.

Artigue, M. (1992). Functions from an algebraic and graphic point of view: Cognitive difficulties and teaching practices. In G. Harel and E. Dubinsky (Eds.), The concept of function: Aspects of epistemology and pedagogy, (pp. 109-132). Washington, DC: Mathematical Association of America.

Baartmans, B. G., \& Sorby, S. A. (1996a). Introduction to 3-d spatial visualization. Englewood Cliffs, NJ: Prentice Hall.

Baartmans, B. G., \& Sorby, S. A. (1996b). Making connections: Spatial skills and engineering drawings. The Mathematics Teacher, 89, 348-357.

Balacheff, N. (1990). Towards a problematique for research on mathematics teaching. Journal for Research in Mathematics Education, 21, 258-272.

Battista, M. T. (1999). The importance of spatial structuring in geometric reasoning. Teaching Children Mathematics, 6, 170-177.

Battista, M. T., Wheatley, G. H., \& Talsma, G. (1982). The importance of spatial visualization and cognitive development for geometry learning in preservice elementary teachers. Journal for Research in Mathematics Education, 27, 258292. 
Ben-Chaim, D., Lappan, G., \& Houang, R. T. (1988). The effects of instruction on spatial visualization of middle boys and girls. American Educational Research Journal, $25,51-71$.

Bennett, D. (1999). Exploring geometry with the geometer's sketchpad. Emeryville, CA: Key Curriculum Press.

Bishop, A. J. (1983). Space and geometry. In R. Lesh \& M. Landau (Eds.), Acquisition of mathematics concepts and processes (pp. 175-203). New York: Academic Press.

Bishop, A. J. (1989). A review of research on visualization in mathematics education. Focus on Learning Problems in Mathematics, 11, 7-16.

Bodner, G. M., \& Guay, R. B. (1997). The Purdue visualization of rotations test. The Chemical Educator, 2, 1-18.

Bouchard, R. J., Jr., \& McGee, M. G. (1977). Sex differences in human spatial ability: Not an x-linked recessive gene effect. Social Biology, 24, 332-335.

Bruner, J. S. (1960). The process of education. Cambridge, MA: Harvard University Press.

Clements, D. H. (2003). Teaching and learning geometry. In J. Kilpatrick, W. G. Martin, and D. Schifter (Eds.), A research companion to principles and standards for school mathematics (pp. 151-178). Reston, VA: National Council of Teachers of Mathematics.

Clements, D. H., \& Battista, M. T. (1990). Constructivist learning and teaching. Arithmetic Teacher, 38, 34-35.

Clements, D. H., \& Battista, M. T. (1992). Geometry and spatial reasoning. In D. A. Grouws (Ed.), Handbook of research on mathematics teaching and learning (pp. 420-464). New York: Macmillan.

Cobb, P. (2000). Conducting teaching experiments in collaboration with teachers. In A. E. Kelley \& R. A. Lesh (Eds.), Handbook of research design in mathematics and science education (pp. 307-334). Mahwah, NJ: Lawrence Erlbaum Associates.

Cobb, P., \& Steffe, L. P. (1983). The constructivist researcher as a teacher and model builder. Journal for Research in Mathematics Education, 14, 83-94.

Cognitive Tutor. (2004). Cognitive tutor: Geometry. Pittsburgh, PA: Carnegie Learning. 
Cohen, J. (1988). Statistical power analysis for the behavioral sciences. New York: Academic Press.

Cook, T. D., \& Campbell, D. T. (1979). Quasi-experimentation: design and analysis issues for field settings. Chicago: Rand McNally College Publishing Company.

Cottrill, J. (2003). An overview of theories of learning in mathematics education research. [Online]. Available: http://homepages.ohiodominican.edu/ cottrillj/ theory-pmet.pdf

Del Grande, J. J. (1987). Spatial perception and primary geometry. In M. M. Lindquist (Ed.), Learning and teaching geometry, $k-12$ (pp. 127-135). Reston, VA: National Council of Teachers of Mathematics.

Delpit, L. (1995). Other people's children: Cultural conflict in the classroom. New York: The New York Press.

Deno, J. A. (1995). The relationship of previous spatial visualization ability. Engineering Design Graphics Journal, 59, 5-17.

Dixon, J. K. (1997). Computer use and visualization in students' construction of reflection and rotation concepts. School Science and Mathematics, 97, 352358.

Dreyfus, T. (1990). Advanced mathematical thinking. In P. Nesher and J. Kilpatrick (Eds.), Mathematics and cognition (pp. 113-134). Cambridge: Cambridge University Press.

Dreyfus, T. (1991). Advanced mathematical thinking processes. In D. Tall (Ed.), Advanced mathematical thinking (pp. 25-41). Dordrecht, The Netherlands: Kluwer.

Dubinsky, E., Dautermann, J., Leron, U., \& Zazkis, R. (1994). On learning fundamental concepts of group theory. Educational Studies in Mathematics, 27, 267-305.

Eisenberg, T. A, \& McGinty, L. (1977). On spatial visualization in college students. The Journal of Psychology, 95, 99-104.

Fuys, D., Geddes, D., \& Tischler, R. (1988). The van Hiele model of thinking in geometry among adolescents. Journal for Research in Mathematics Education Monograph 3. Reston, VA: National Council of Teachers of Mathematics.

Gillespie, W. H. (1995). Using solid modeling tutorial to enhance visualization skills. Unpublished doctoral dissertation, University of Idaho, Moscow, ID. 
Glaser, B. G., \& Strauss, A. L. (1967). The discovery of grounded theory: Strategies for qualitative research. Chicago: Aldine.

Goldin, G. A. (1987). Cognitive representational systems for mathematical problem solving. In C. Janvier (Ed.), Problems of representation in the teaching and learning of mathematics (pp. 125-145). Hillsdale, NJ: Erlbaum.

Goldin, G. A. (2003). Representation in school mathematics: A unifying research perspective. In J. Kilpatrick, W. G. Martin, and D. Schifter (Eds.), A research companion to principles and standards for school mathematics (pp. 275-285). Reston, VA: National Council of Teachers of Mathematics.

Goldin, G. A., Kaput, J. J. (1996). A joint perspective on the idea of representation in learning and doing mathematics. In L. Steffe \& P. Nesher (Eds.), Theories of mathematical learning (pp. 397-430). Mahwah, NJ: Lawrence Erlbaum Associates.

Gorgorio, N. (1998). Exploring the functionality of visual and non-visual strategies in solving rotation problems. Educational Studies in Mathematics, 35, 207-231.

Greene, J. C., Caracelli, V. J., \& Graham, W. F. (1989). Toward a conceptual framework for mixed-method evaluation designs. Educational Evaluation and Policy Analysis, 11, 255-274.

Guay, R. (1977). Purdue spatial visualization tests. Purdue Research Foundation, West Lafayette, $\mathbb{I N}$.

Gutierrez, A. (1996). Visualization in three-dimensional geometry: In search of a framework. Proceedings of the 20th PME International Conference, Spain, 1, $1-19$.

Gutierrez, A., Jaime, A., \& Fortuny, J.M. (1991). An alternative paradigm to evaluate the acquisition of the van Hiele levels. Journal for Research in Mathematics Education, 22, 237-251.

Hamilton, C. J. (1995). Beyond sex differences in visuo-spatial processing: The impact of gender trait possession. British Journal of Psychology, 86, 1-20.

Hershkowitz, R. (1990). Psychological aspects of learning geometry. In P. Nesher \& J. Kilpatrick (Eds.), Mathematics and cognition (pp. 70-95). New York: Cambridge University Press.

Hoz, R. (1981). The effects of rigidity on school geometry learning. Educational Studies in Mathematics, 12, 171-190. 
Jackiw, N. (1995). The geometer's sketchpad: User guide and reference manual. Berkeley, CA: Key Curriculum Press.

July, R. A. (2001). Thinking in three dimensions: Exploring students' geometric thinking and spatial ability with the geometer's sketchpad. Unpublished doctoral dissertation, Florida International University, Miami, FL.

Kantowski, E. L. (1974). Processes in mathematical problem solving. Unpublished doctoral dissertation, University of Georgia, Athens, GA.

Kaput, J. J. (1987). Representational systems and mathematics. In C. Janvier (Ed.), Problems of representation in the teaching and learning of mathematics (pp. 1926). Hillsdale, NJ: Erlbaum.

Kaput, J. J. (1998). Representations, inscriptions, descriptions and learning: A kaleidoscope of windows. Journal of Mathematical Behavior, 17, 265-281.

Kilpatrick, J., \& Wirszup, I. (Eds.). (1978). Soviet studies in the psychology of learning and teaching mathematics: The development of spatial abilities, 5. Stanford, CA: School Mathematics Study Group.

Kruteskii, V. A. (1976). The psychology of mathematical abilities in schoolchildren. Chicago: The University of Chicago Press.

Lappan, G. (1999). Geometry: the forgotten strand. NCTM News Bulletin, 36(5), 3.

Linn, M. C. \& Petersen, A. C. (1985). Emergence and characterization of sex differences in spatial ability: A meta-analysis. Child Development, 56, 1479-1498.

McGee, M. G. (1979). Human spatial abilities. New York: Praeger Publishers.

Miles, M. B., \& Huberman, A. M. (1984). Qualitative data analysis: An expanded sourcebook. Thousand Oaks, CA: Sage Publications.

Mullis, I. V. S., Martin, M. O., Beaton, A. E., Gonzalez, E. J., Kelly, D. L., \& Smith, T. A. (1997). Mathematics achievement in the primary school years: IEA's third International Mathematics and Science Study (TIMSS). Chestnut Hill, MA: Boston College, Center for the Study of Testing, Evaluation, and Educational Policy.

National Council of Teachers of Mathematics. (1989). Curriculum and evaluation standards for school mathematics. Reston, VA: National Council of Teachers of Mathematics. 
National Council of Teachers of Mathematics. (2000). Principles and standards for school mathematics. Reston, VA: National Council of Teachers of Mathematics.

Owens, K. D., \& Clements, M. A. (1998). Representations used in spatial problem solving in the classroom. Journal of Mathematical Behavior, 17, 197-218.

Paivio, A. (1991). Imagery and verbal processes. New York: Holt, Rinehart \& Winston.

Papert, S. (1980). Mindstorms: Children, computers, and powerful ideas. New York: Basic Books.

Papert, S. (1993). The children's machine: Rethinking school in the age of the computer. New York: Basic Books.

Pitta-Pantazi, D., Gray, E., \& Christou, C. (2004). Elementary school students mental representations of fractions. Proceedings of the 28th PME International Conference, Norway, 4, 41-48.

Polya, G. (1957). How to solve it. Princeton, NJ: Princeton University Press.

Polydron International. (2003). Polydron class kit. Gloucestershire, Great Britain: Polydron International.

Presmeg, N. C. (1986). Visualization in highschool mathematics. For the Learning of Mathematics, 6, 42-46.

Presmeg, N. C. (2006). Research on visualization in learning and teaching mathematics: emergence from psychology. In A. Gutierrez and P. Boero (Eds.), Handbook of research on the psychology of mathematics education, (pp. 205-235). Rotterdam: Sense Publishers.

Rensink, R. A. (2000). The dynamic representation of scenes, Visual Cognition 7, 17-42.

Rossman, G. B., \& Rallis, S. F. (2003). Learning in the field: An introduction to qualitative research. London: Sage Publications.

Salkind, N. J. (1976). A cross-dimensional study of spatial visualization in young children. Journal of Genetic Psychology, 129, 339-340.

Schoenfeld, A. H. (2002). Research methods in (mathematics) education. In L. D. English (Ed.), Handbook of international research in mathematics education (pp. 435-487). Mahwah, NJ: Lawrence Erlbaum Associates.

Senk, S. L. (1985). How well do students write geometry proofs? Mathematics Teacher, $78,448-456$. 
Serra, M. (1993). Discovering geometry: An inductive approach. Berkeley, CA: Key Curriculum Press.

Sexton, T. J. (1992). Effect on spatial visualization: Introducing basic engineering graphics concepts using CAD technology. Engineering Design Graphics Journal, $56,36-43$.

Shepard, R. N., \& Metzler, J. (1971). Mental rotation of three-dimensional objects. Science, 171, 701-703.

Sinclair, M. (2004). Working with accurate representations: The case of preconstructed dynamic geometry sketches. Journal of Computers in Mathematics and Science Teaching, 23, 191-208.

Strong, S., \& Smith, R. (2002). Spatial visualization: Fundamentals and trends in engineering graphics. Journal of Industrial Technology, 18, 1-6.

Usiskin, S. (1982). van Hiele levels and achievement in secondary school geometry. Cognitive Development and Achievement in Secondary School Geometry Project. Chicago: The University of Chicago.

Vandenberg, S. G., \& Kuse, A. R. (1978). Mental rotations, a group test of threedimensional spatial visualization. Perceptual \& Motor Skills, 47, 599- 604.

van Hiele, P. M. (1986). Structure and insight: A theory of mathematics education. Orlando, FL: Academic Press.

van Hiele-Geldof, D. (1984). The didactics of geometry in the lowest class of secondary school. In D. Fuys, D. Geddes, \& R. Tischler (Eds.), English translation of selected writings of Dina van Hiele-Geldof and Pierre M. van Hiele (pp. 1-214). Brooklyn, NY: Brooklyn College, School of Education. (ERIC Document Reproduction Service No. 289 697)

von Glasersfeld, E. (1995). Radical constructivism: $A$ way of knowing and learning. London: Falmer Press.

von Glasersfeld, E. (1996). Aspects of radical constructivism and its educational recommendations. In L. Steffe \& P. Nesher (Eds.), Theories of mathematical learning (pp. 307-314). Mahwah, NJ: Lawrence Erlbaum Associates.

Vygotsky, L. S. (1978). Mind in society: The development of higher psychological processes. Cambridge, MA: Harvard University Press.

Vygotsky, L. S. (1986). Thought and language. Cambridge, MA: MIT Press. 
Wheatley, G. H. (1990). Spatial sense and mathematics learning. Arithmetic Teacher, $37,10-11$.

White, P., \& Mitchelmore, M. (2003). Teaching angles by abstraction from physical activities with concrete materials. Proceedings of the 27th PME International Conference, USA , 4, 403-410.

Woolner, P. (2004). A comparison of a visual-spatial approach and a verbal approach to teaching mathematics. Proceedings of the 28th PME International Conference, Norway, 4, 449-456.

Yen, W. M. (1975). Sex-linked major-gene influences on selected types of spatial performance. Behavior Genetics, 5, 281-298.

Zazkis, R., Dubinsky, E., \& Dautermann, J. (1996). Coordinating visual and analytic strategies. Journal for Research in Mathematics Education, 27, 435-457. 


\section{APPENDIX A}

Problems in Lessons \#1 - \#6

Lesson \#1. Algebraic Expressions

These problems ask students to write algebraic expressions which describe the geometric structure of solids.

1. Select a cube from the color blocks and attach other cubes to each of the faces of the original cube.

a. Make a sketch of the new solid. Write a sentence describing its geometric structure.

b. Write an algebraic expression representing its surface area.

c. Write an algebraic expression representing its volume.

d. Using a ratio, compare the surface area of the original cube with the surface area of the new solid. Similarly, compare the volume of the original cube with the volume of the new solid.

2. Repeat problem number 1 , but start with a tetrahedron constructed with Polydron pieces.

a. Make a sketch of the new solid. Write a sentence describing its geometric structure.

b. Write an algebraic expression representing its surface area.

c. Write an algebraic expression representing its volume.

d. Using a ratio, compare the surface area of the original tetrahedron with the surface area of the new solid. Similarly, compare the volume of the original tetrahedron with the volume of the new solid.

3. Using the pentagonal dipyramid constructed with Polydron pieces:

a. Write an algebraic expression representing its surface area. 
b. Write an algebraic expression representing its volume.

4. Create a solid of your own and analyze it using questions $\mathrm{a}, \mathrm{b}$, and $\mathrm{c}$ from problem 1.

\section{Lesson \#2. Dilation Theorems}

With these problems students investigate the effect of altering the dimensions of solids. By using proportionality and exponentiation, students are able to establish how the surface area and volume of solids change.

1. Construct a cube with the Polydron pieces. Double the sides of each square in the cube. What is the ratio between the surface areas of the original cube and the new cube? What is the ratio between the volumes of the original cube and the new cube?

2. Repeat problem number 1 , but start with a tetrahedron.

3. How many tetrahedra make up the volume of one octahedron? How did you determine this?

4. Given any solid, if you enlarge its dimensions five times, how will its volume change?

Lesson \#3. Measurement.

Students use these problems to calculate different lengths of solids. They compare these calculations with actual measurements.

1. What is the length of the diagonal of a cube of:

a. Side, $\mathrm{s}=1$ ?

b. Side, $\mathrm{s}=5$ ?

(Hint: Find the length of the diagonal of a square first).

2. Construct a rectangular right prism with the Polydron pieces using two squares and four rectangles (the rectangles are the lateral faces and the squares are the top and bottom 
faces). What is the length of its diagonal? (Hint: you need to determine the dimensions of the rectangles. Assume the square is of side $s=1$ and think about slicing the unit cube in order to get a rectangular cross-section).

3. A penta-hut is an open three-dimensional figure made of four regular pentagons. All four pentagons meet at one vertex creating a saddle-point (see Figure 19 in Appendix B).

Fit a right isosceles triangle between two pentagons at each end of the penta-hut whose pentagons are of:

a. Side, $s=6$. Find the length of the triangle's hypotenuse.

b. Side, $s=\sqrt{6}$. Find the length of the triangle's hypotenuse.

4. What is the height of:

a. An octahedron of side, $s=1$ ?

b. A tetrahedron of side, $s=1$ ? (Hint: the height is defined as the distance from the centroid of the base triangle to that base's opposite vertex, and the centroid of an equilateral triangle is located at $\frac{1}{3}$ of its height with respect to its base).

5. Given a cube of side $s=3$, find the sum of the lengths of two cube diagonals plus two cube edges.

Lesson \#4. Duality of Platonic Solids

Students answer several questions after constructing in GSP models of the five Platonic solids.

1. Construct a cube in GSP.

2. Construct a tetrahedron in GSP.

3. Construct an octahedron in GSP. 
4. Construct a dodecahedron in GSP.

5. Construct an icosahedron in GSP.

6. Using the constructions of a cube and a tetrahedron from problems 1 and 2 above, construct two octahedra. Using the cube, connect the centers of all squares to construct the first octahedron. Similarly, using the tetrahedron, connect the midpoints of all edges to construct the second octahedron.

7. Make a chart showing the number of vertices, edges, and faces for each of the Platonic solids.

a. Discover an equation which relates the number of vertices, edges, and faces. The equation is true for all Platonic solids.

b. Identify the dual of each Platonic solid.

8. Describe the shape of a cross-section through five vertices of an icosahedron.

\section{Lesson \#5. Coordinate Geometry}

Using a prototype of Figure 13 in chapter four as a guide, students construct the solid in GSP. Students are allowed to use the cube constructed in lesson \#4 to start. The purpose of these problems is to familiarize students with three-dimensional coordinates. 1. Using GSP, construct and shade two right triangles which will allow you to apply the Pythagorean theorem twice in order to determine the length of segment $A B$.

2. What is the length of segment $A B$ ?

3. Choose a vertex as the origin and label it with coordinates $(0,0,0)$.

4. Starting from the origin, label the $x-, y-$, and $z$-axes.

5. What are the coordinates of points $\mathrm{A}$ and $\mathrm{B}$ ?

6. Use the distance formula to find the length of segment $A B$. 
In this lesson, students solve three-dimensional problems by first formulating analogous questions for equivalent two-dimensional problems.

1. Six congruent square right pyramids constructed inside each face of a cube intersect inside the cube if the pyramid heights are larger than half the height of the cube.

a. Visualize the solid thus formed and describe its shape in one or two sentences.

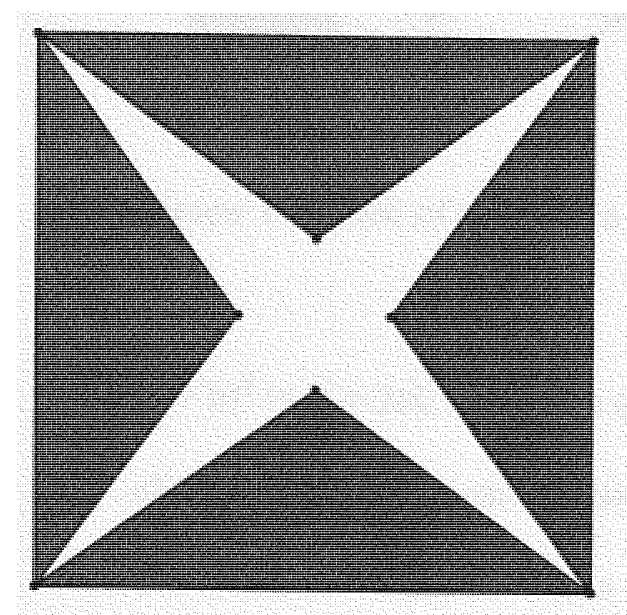

Figure 16. Two-dimensional analogy for problem \#1.

b. Making reference to Figure 16, formulate the two-dimensional analogy for problem 1 [Hint: Use a square instead of a cube and a triangle (what type?) instead of a pyramid].

c. Construct with the Polydron pieces the solid formed by the intersecting pyramids of problem 1.

2. Use the Polydron pieces to help you visualize $a^{3}+3 a b^{2}+3 a^{2} b+b^{3}$, the algebraic expression for the expansion of $(a+b)^{3}$. 
a. Construct a cube of side $s=a+b$ with the Polydron pieces. Use a cube made of six squares to represent the term $\mathrm{a}^{3}$ and let $\mathrm{b}=2 \mathrm{a}$. Recall that the expansion of

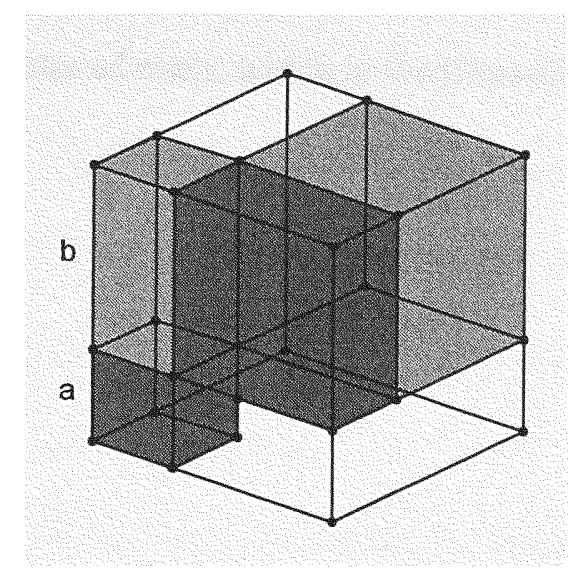

Figure 17. Cube of side, $\mathrm{s}=\mathrm{a}+\mathrm{b}$.

$(a+b)^{2}=a^{2}+2 a b+b^{2}$. This quadratic expansion is the two-dimensional analogy of the original problem. Figure 18 is a two-dimensional analogy of Figure 17, which shows a cube of side, $s=a+b$, and four of eight prisms representing the terms in the cubic expansion.

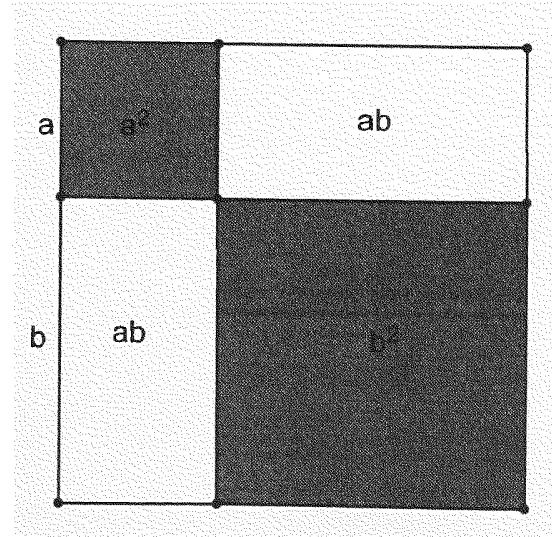

Figure 18. Two-dimensional analogy for problem \#2. 

b. Expand $(a+b)^{5}$.

3. The centroid of an equilateral triangle is located at $1 / 3$ of the altitude, starting from the base to the opposite vertex. For a regular tetrahedron, find the location of the center of mass, which is the three-dimensional analogy of the centroid. 


\section{APPENDIX B}

\section{Complementary Problems}

These problems are used as complementary problems to the ones in lessons \#1-6. Students who finish early can continue working on these problems.

1. Find the heights of the following solids.

a. A tetrahedron with $\mathrm{s}=1$.

b. An octahedron with $s=2$ (The height is the distance between two opposite vertices).

2. Using $\mathrm{T}$ for the volume of a tetrahedron $(\mathrm{s}=1)$, and $\mathrm{P}$ for the volume of a regular square pyramid $(\mathrm{s}=1)$, express algebraically the volume for a cuboctahedron ( $\mathrm{s}=$ 1). Recall that a cuboctahedron is constructed by slicing off eight triangular pyramids from a cube. The triangular pyramids are formed by connecting the midpoints of three converging edges of a cube.

4. What solid has a larger volume: a cube of $s=4$ or an octahedron of $s=5$ ? Explain why.

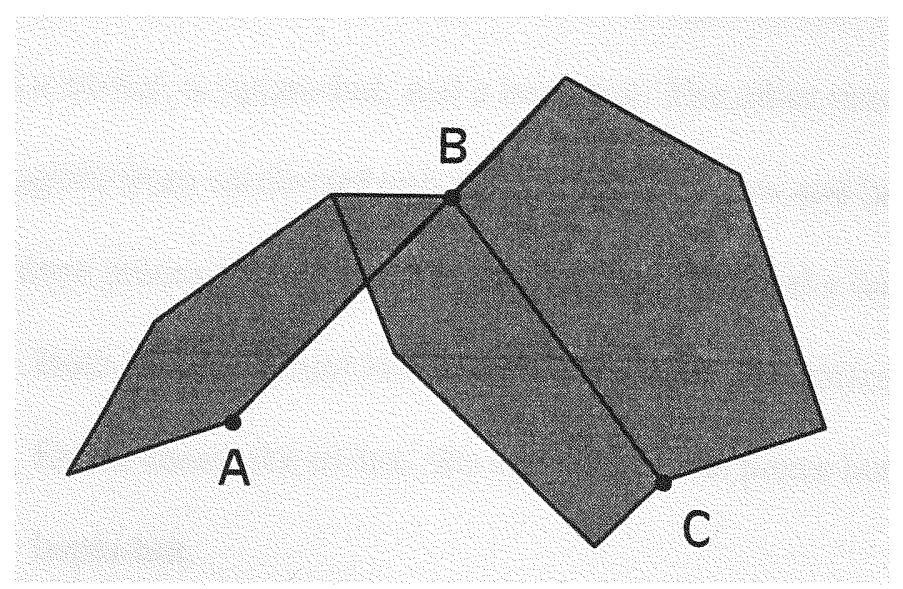

Figure 19. The penta-hut. 
5. The geometric object made of four regular pentagons shown in Figure 19 is called a penta-hut. Use the Polydron pieces to construct the penta-hut, and place it on a flat surface so that one edge of each pentagon is on the surface. What is your conjecture about the measure of angle $\mathrm{ABC}$ ?

6. Separate the penta-hut into two halves through edges $A B$ and $B C$. In each pair of pentagons, insert in angle $A B C$ each of the following pieces, one at a time: an equilateral triangle, a square, a regular pentagon, and a regular hexagon. Bring both halves together to re-assemble the penta-hut. Which of the four situations differs from the other three? Why?

7. What should be the measure of angle $A B C$ so that if you flip the penta-hut upside down it would preserve its shape?

8. Why do you think point B is called a "saddle-point"?

9. Build a class of objects resembling the penta-hut, but made of four triangles, four squares, four hexagons, or four heptagons. We will call these tri-hut, square-hut, hexa-hut, and hepta-hut. Two groups of students will cut four obtuse triangles from cardboard and will use squares and hexagons from the Polydron pieces to form a tri-hut, a square-hut, and a hexa-hut. The other two groups will cut two heptagons from cardboard and will also use squares and hexagons from the Polydron pieces to form a square-hut, a hexa-hut, and a hepta-hut.

a. Both groups should list four mathematical generalizations that can be made for all cases: the tri-hut, the square-hut, the penta-hut, the hexa-hut, and the hepta-hut. 
b. If four octagons are assembled into an octa-hut resting on a flat surface, and each octagon has one side touching the surface, what will be the measure of angle $\mathrm{ABC}$ ? 
APPENDIX C

Forms for the Collection of Qualitative Data

\section{Protocol Problems}

Name:

Date:

Protocol Problem \#

Problem Statement:

Answer:

II. Mathematical Truths

III. Sketch

IV. Explain 
Interviews

Name:

Date:

Problem \#

Questions.

Visual Thinking Questions

V1. What visual image (mental picture) can you use to solve this problem?

V2. Depends on answer to V1.

V3. Depends on answers to V1 and V2.

Analytic Thinking Questions

A1. What math theorem/formula/relation can you use to solve this problem?

A2. Depends on answer to A1.

A3. Depends on answers to A1 and A2.

Cognitive Processes.

$\square$ Switching from $V *$ to $A *$ reasoning Switching from $\mathrm{A}$ to $\mathrm{V}$ reasoning

$\square$ Connecting to prior $\mathrm{V}$ knowledge Connecting to prior A knowledge

$\square$ Other:

* $\mathrm{V}=$ visual, $\mathrm{A}=$ analytic

Comments: 
Whole-Part Mental Processes.

In addition to the four cognitive processes listed above, nine other mental processes were recorded. These processes are mental manipulations of three types of three-dimensional representations students use: original mental images, images based on GSP constructions, or images externalized on paper sketches. The transformation processes refer to objects perceived as a whole, whereas the action processes refer to objects perceived as a composition of parts.

Transformations

$\square$ Rotate (ROT)

$\square$ Reflect (REF)

$\square$ Translate (TRA)

$\square$ Dilate (DIL)

\section{Actions}

$\square$ slice (SLI)

$\square$ Fold (FOL)

$\square$ Connect vertices (CON)

$\square$ Construct intersection (INT)

$\square$ Combine \& Separate (C\&S) 
GSP Problems

GSP Problem \#

\begin{tabular}{|c|c|c|c|}
\hline $\begin{array}{c}\text { Strategy } \\
\text { Type }\end{array}$ & $\begin{array}{c}\text { Number of } \\
\text { Strategy Types } \\
\text { (Fully Constructing) } \\
\end{array}$ & $\begin{array}{c}\text { Number of } \\
\text { Strategy Types } \\
\text { (Partially Constructing) }\end{array}$ & Description of Strategy \\
\hline A & 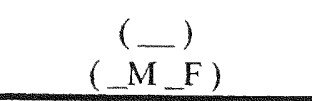 & $\left({ }^{(}{ }_{\mathrm{M}} \mathrm{F}\right)$ & \\
\hline B & $\left(\begin{array}{c}(-) \\
(\mathrm{M}-\mathrm{F}) \\
\end{array}\right.$ & $\left(\frac{(-)}{M}\right)$ & \\
\hline $\mathrm{C}$ & $\left.(\stackrel{(-)}{M})^{-}\right)$ & $(\stackrel{(-)}{M} \mathrm{~F})$ & \\
\hline $\mathrm{D}$ & 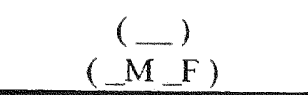 & 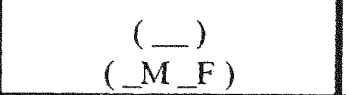 & \\
\hline $\mathrm{E}$ & $\left(\begin{array}{l}(-) \\
M\end{array}\right)$ & $\left(\begin{array}{l}(\bar{M}) \\
(\mathrm{F})\end{array}\right.$ & \\
\hline $\mathrm{F}$ & $\begin{array}{c}(\bar{G}) \\
(\mathrm{M}-\mathrm{F}) \\
\end{array}$ & $(\stackrel{(-)}{M} \mathrm{~F})$ & \\
\hline G & $\left(\begin{array}{c}(-) \\
M-F\end{array}\right)$ & $\begin{array}{c}(\bar{C}) \\
(\mathrm{M}-\mathrm{F})\end{array}$ & \\
\hline & $\underline{-}$ & $\ldots$ & Total number of students \\
\hline
\end{tabular}

*-.- $\left(\mathrm{M}_{-} \mathrm{F}\right)$ is used to report number of males and females working together. 
Field Notes

Day \#

Label:

Period:

Context:

Problem:

Observations:

Checklist for whole-part mental processes

\begin{tabular}{|l|l|l|l|l|l|l|l|l|l|}
\hline Day & C\&S & TRA & DIL & ROT & REF & SLI & FOL & CON & INT \\
\hline & & & & & & & & & \\
\hline
\end{tabular}

Day \#

Label:

Period:

Context:

Problem:

Observations:

Checklist for whole-part mental processes

\begin{tabular}{|l|l|l|l|l|l|l|l|l|l|}
\hline Day & C\&S & TRA & DIL & ROT & REF & SLI & FOL & CON & INT \\
\hline & & & & & & & & & \\
\hline
\end{tabular}




\section{APPENDIX D}

Rubric for Process-Product Score

Process Score

Recall

Recalls relevant information, visual or analytic.......... 1.0 point

Recalls irrelevant information or leaves blank............ 0.0 points

Sketch (Form Mental Image)

Effectively uses a 3-D sketch* as part of the solution..... 1.0 point Partially uses a relevant 3-D sketch* or

a 2-D sketch* which codes 3-D information........ 0.5 point

Uses a weak sketch*, irrelevant sketch*or leaves blank.....0.0 points

*--During the protocol problems students were assessed on

how well they could sketch, whereas in the interviews they

were assessed on how well they could form mental images.

Explain by Writing or Numerical Solution

Uses clear, complete, sound logic...................... 1.0 point

Partially uses sound logic ............................... 0.7 point

Uses weak logic, insipient start or visual estimate.......... 0.3 point

Uses non-related logic or leaves blank .................. 0.0 points

Product Score

Answer

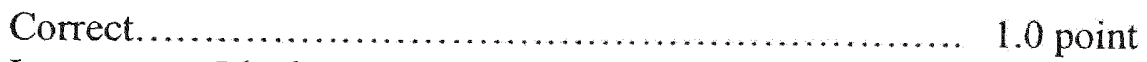

Incorrect or Blank ................................ 0.0 points

Maximum Score: 4.0 points 


\section{APPENDIXE}

PSVT Results for Present Dissertation Study

Table E1

Control Group

\begin{tabular}{|c|c|c|c|c|c|c|c|c|c|}
\hline \multirow[b]{2}{*}{ No. } & \multirow[b]{2}{*}{ Gender } & \multicolumn{4}{|c|}{ Pretest } & \multicolumn{4}{|c|}{ Posttest } \\
\hline & & PreTot & DEV & ROT & VIE & PostTot & $\overline{D E V}$ & ROT & VIE \\
\hline 1 & 0 & 15 & 6 & 3 & 6 & 15 & 7 & 4 & 4 \\
\hline 2 & 0 & 15 & 7 & 4 & 4 & 13 & 5 & 5 & 3 \\
\hline 3 & 1 & 26 & 11 & 8 & 7 & 25 & 10 & 8 & 7 \\
\hline 4 & 1 & 4 & 1 & 1 & 2 & 12 & 5 & 6 & 1 \\
\hline 5 & 1 & 18 & 1 & 8 & 9 & 27 & 5 & 11 & 11 \\
\hline 6 & 0 & 23 & 9 & 5 & 9 & 14 & 4 & 4 & 6 \\
\hline 7 & 0 & 11 & 4 & 3 & 4 & 13 & 5 & 2 & 6 \\
\hline 8 & 0 & 17 & 3 & 8 & 6 & 18 & 10 & 5 & 3 \\
\hline 9 & 0 & 14 & 4 & 5 & 5 & 15 & 3 & 8 & 4 \\
\hline 10 & 0 & 9 & 2 & 3 & 4 & 14 & 7 & 2 & 5 \\
\hline 11 & 1 & 25 & 7 & 8 & 10 & 22 & 5 & 7 & 10 \\
\hline 12 & 1 & 13 & 6 & 4 & 3 & 23 & 8 & 6 & 9 \\
\hline 13 & 1 & 5 & 1 & 3 & 1 & 6 & 3 & 2 & 1 \\
\hline 14 & 0 & 6 & 4 & 2 & of & 9 & 3 & 2 & 4 \\
\hline 15 & 1 & 17 & 4 & 6 & 7 & 28 & 7 & 11 & 10 \\
\hline 16 & 0 & 6 & 3 & 2 & 1 & 9 & 6 & 2 & 1 \\
\hline 17 & 0 & 6 & 2 & 2 & 2 & 11 & 5 & 5 & 1 \\
\hline 18 & 0 & 21 & 7 & 3 & 11 & 28 & 11 & 6 & 11 \\
\hline 19 & 0 & 16 & 4 & 3 & 9 & 16 & 4 & 5 & 7 \\
\hline 20 & 0 & 11 & 2 & 5 & 4 & 14 & 2 & 8 & 4 \\
\hline 21 & 1 & 7 & 3 & 1 & 3 & 17 & 3 & 5 & 9 \\
\hline
\end{tabular}

Note. Female $=0$ and Male $=1$. 
Table E2

Fully Constructing Models with GSP (Experimental Group 1)

\begin{tabular}{|c|c|c|c|c|c|c|c|c|c|}
\hline \multirow[b]{2}{*}{ No. } & \multirow[b]{2}{*}{ Gender } & \multicolumn{4}{|c|}{ Pretest } & \multicolumn{4}{|c|}{ Posttest } \\
\hline & & PreTot & $\mathrm{DEV}$ & ROT & VIE & PostTot & $\overline{D E V}$ & ROT & VIE \\
\hline 1 & 1 & 26 & 6 & 11 & 9 & 32 & 12 & 10 & 10 \\
\hline 2 & 1 & 13 & 5 & 4 & 4 & 18 & 9 & 3 & 6 \\
\hline 3 & 1 & 12 & 6 & 4 & 2 & 24 & 11 & 5 & 8 \\
\hline 4 & 0 & 12 & 7 & 1 & 4 & 19 & 10 & 5 & 4 \\
\hline 5 & 0 & 12 & 3 & 6 & 3 & 9 & 4 & 4 & 1 \\
\hline 6 & 0 & 14 & 4 & 3 & 7 & 22 & 9 & 9 & 4 \\
\hline 7 & 1 & 11 & 2 & 4 & 5 & 16 & 5 & 3 & 8 \\
\hline 8 & 1 & 18 & 4 & 6 & 8 & 14 & 2 & 3 & 9 \\
\hline 9 & 0 & 16 & 5 & 4 & 7 & 12 & 3 & 3 & 6 \\
\hline 10 & 0 & 5 & 1 & 2 & 2 & 11 & 3 & 5 & 3 \\
\hline 11 & 0 & 7 & 2 & 4 & 1 & 8 & 2 & 5 & 1 \\
\hline 12 & 1 & 13 & 3 & 6 & 4 & 18 & 5 & 7 & 6 \\
\hline 13 & 1 & 16 & 6 & 6 & 4 & 12 & 5 & 4 & 3 \\
\hline 14 & 0 & 13 & 4 & 3 & 6 & 16 & 9 & 4 & 3 \\
\hline 15 & 1 & 10 & 2 & 1 & 7 & 19 & 4 & 7 & 8 \\
\hline 16 & 0 & 7 & 3 & 0 & 4) & 6 & 3 & 1 & 2 \\
\hline 17 & 1 & 14 & 3 & 7 & 4 & 11 & 4 & 2 & 5 \\
\hline 18 & 0 & 17 & 8 & 5 & 4 & 22 & 8 & 5 & 9 \\
\hline 19 & 0 & 18 & 9 & 3 & 6 & 17 & 9 & 6 & 2 \\
\hline 20 & 0 & 30 & 10 & 11 & 9 & 34 & 12 & 11 & 11 \\
\hline 21 & 0 & 14 & 5 & 4 & 5 & 11 & 6 & 2 & 3 \\
\hline 22 & 1 & 20 & 5 & 7 & 81 & 21 & 5 & 7 & 9 \\
\hline
\end{tabular}

Note. Female $=0$ and Male $=1$. 
Table E3

Partially Constructing Models with GSP (Experimental Group 2)

\begin{tabular}{|c|c|rrrr|rrrr|r|}
\hline \multirow{2}{*}{ No. } & Gender & \multicolumn{5}{|c|}{ Pretest } & \multicolumn{4}{|c|}{ Posttest } \\
\hline 1 & 0 & 11 & 4 & 4 & 3 & 15 & 6 & 5 & 4 \\
2 & 0 & 14 & 5 & 7 & 2 & 13 & 3 & 5 & 5 \\
3 & 0 & 6 & 1 & 1 & 4 & 13 & 4 & 4 & 5 \\
4 & 0 & 13 & 5 & 2 & 6 & 13 & 5 & 5 & 3 \\
5 & 0 & 8 & 1 & 3 & 4 & 16 & 6 & 2 & 8 \\
6 & 1 & 15 & 7 & 3 & 5 & 24 & 9 & 4 & 11 \\
7 & 0 & 19 & 8 & 5 & 6 & 24 & 9 & 7 & 8 \\
8 & 0 & 18 & 6 & 6 & 6 & 21 & 9 & 5 & 7 \\
9 & 1 & 7 & 2 & 0 & 5 & 9 & 3 & 2 & 4 \\
10 & 1 & 17 & 4 & 5 & 8 & 18 & 6 & 4 & 8 \\
11 & 1 & 7 & 5 & 0 & 2 & 9 & 4 & 2 & 3 \\
12 & 1 & 22 & 5 & 6 & 11 & 26 & 7 & 9 & 10 \\
13 & 0 & 11 & 5 & 3 & 3 & 11 & 2 & 6 & 3 \\
14 & 0 & 14 & 3 & 5 & 6 & 18 & 5 & 7 & 6 \\
15 & 1 & 9 & 4 & 1 & 4 & 10 & 4 & 3 & 3 \\
16 & 0 & 4 & 0 & 3 & 1 & 5 & 1 & 3 & 1 \\
17 & 1 & 12 & 5 & 5 & 2 & 10 & 4 & 1 & 5 \\
18 & 0 & 11 & 4 & 3 & 4 & 15 & 9 & 4 & 2 \\
19 & 1 & 21 & 7 & 7 & 7 & 25 & 9 & 10 & 6 \\
20 & 0 & 13 & 3 & 4 & 6 & 19 & 5 & 6 & 8 \\
21 & 1 & 17 & 2 & 8 & 7 & 25 & 9 & 9 & 7 \\
\hline
\end{tabular}

Note. Female $=0$ and Male $=1$. 


\section{APPENDIX F}

Development of Sketching Ability Using Process-Product Score

Sketching Process Score for Protocol Problems \#I and \#2

\begin{tabular}{|c|c|c|c|c|}
\hline & \multicolumn{2}{|c|}{ W. Gender } & \multicolumn{2}{|c|}{ 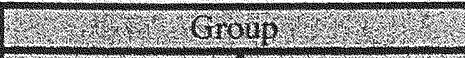 } \\
\hline & Male & Female & Ex: Tuly & Ponrially \\
\hline \multirow{4}{*}{$\begin{array}{c}P \\
R \\
O \\
B\end{array}$} & $\begin{array}{l}0.00 \\
0.00 \\
0.00\end{array}$ & & $\begin{array}{l}0.00 \\
0.00 \\
0.00\end{array}$ & \\
\hline & & $\begin{array}{l}1.00 \\
0.00 \\
0.00\end{array}$ & $\begin{array}{l}1.00 \\
0.00 \\
0.00\end{array}$ & \\
\hline & $\begin{array}{l}0.00 \\
0.00 \\
0.00 \\
\end{array}$ & & & $\begin{array}{l}0.00 \\
0.00 \\
0.00\end{array}$ \\
\hline & & $\begin{array}{l}0.00 \\
0.50 \\
0.50 \\
\end{array}$ & & $\begin{array}{l}0.00 \\
0.50 \\
0.50 \\
\end{array}$ \\
\hline \multirow{4}{*}{$\begin{array}{c}\mathrm{P} \\
\mathrm{R} \\
\mathrm{O} \\
\mathrm{B}\end{array}$} & $\begin{array}{l}0.50 \\
0.50 \\
0.00\end{array}$ & & $\begin{array}{l}0.50 \\
0.50 \\
0.00\end{array}$ & \\
\hline & & $\begin{array}{l}1.00 \\
0.00 \\
0.00 \\
\end{array}$ & $\begin{array}{l}1.00 \\
0.00 \\
0.00\end{array}$ & \\
\hline & $\begin{array}{l}0.00 \\
0.00 \\
0.00 \\
\end{array}$ & & & $\begin{array}{l}0.00 \\
0.00 \\
0.00\end{array}$ \\
\hline & & $\begin{array}{l}0.00 \\
0.00 \\
0.50 \\
\end{array}$ & & $\begin{array}{l}0.00 \\
0.00 \\
0.50 \\
\end{array}$ \\
\hline Score per Problem & 0.50 & 1.75 & 1.50 & 0.75 \\
\hline Number of Students & 6.00 & 6.00 & 6.00 & 6.00 \\
\hline Score per Student & 0.08 & 0.29 & 0.25 & 0.13 \\
\hline \multicolumn{5}{|c|}{ Rubric } \\
\hline $\begin{array}{l}\text { Low Ability } \\
\text { Medium Ability } \\
\text { High Ability } \\
\end{array}$ & $\begin{array}{l}0.00 \\
0.50 \\
1.00 \\
\end{array}$ & $\begin{array}{l}0.00 \\
0.50 \\
1.00 \\
\end{array}$ & $\begin{array}{l}0.00 \\
0.50 \\
1.00 \\
\end{array}$ & $\begin{array}{l}0.00 \\
0.50 \\
1.00 \\
\end{array}$ \\
\hline Summary & $\begin{array}{l}\text { Cendericrour } \\
\text { Male } \\
\text { Female } \\
\text { Fully } \\
\text { Partially } \\
\end{array}$ & \begin{tabular}{|c|} 
Actual Serore \\
0.08 \\
0.29 \\
0.25 \\
0.13 \\
\end{tabular} & 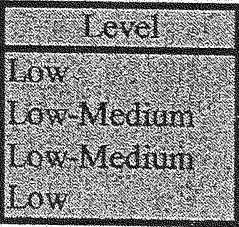 & \\
\hline
\end{tabular}


Sketching Process Score for Protocol Problems \#3 and \#4

\begin{tabular}{|c|c|c|c|c|}
\hline & \multicolumn{2}{|c|}{ 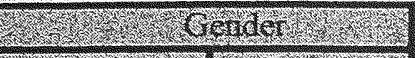 } & \multicolumn{2}{|c|}{ 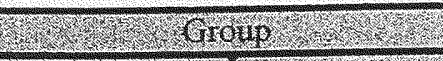 } \\
\hline & Sirvile & Terancie & (2) Tuly & Partally \\
\hline \multirow{4}{*}{$\begin{array}{c}P \\
R \\
O \\
B \\
\# 3\end{array}$} & $\begin{array}{l}0.50 \\
0.50 \\
0.50 \\
\end{array}$ & & $\begin{array}{l}0.50 \\
0.50 \\
0.50\end{array}$ & \\
\hline & & $\begin{array}{l}1.00 \\
0.50 \\
0.00\end{array}$ & $\begin{array}{l}1.00 \\
0.50 \\
0.00\end{array}$ & \\
\hline & $\begin{array}{l}0.50 \\
0.00 \\
0.50 \\
\end{array}$ & & & $\begin{array}{l}0.50 \\
0.00 \\
0.50\end{array}$ \\
\hline & & $\begin{array}{l}0.50 \\
0.50 \\
0.50\end{array}$ & & $\begin{array}{l}0.50 \\
0.50 \\
0.50\end{array}$ \\
\hline \multirow{4}{*}{$\begin{array}{c}\mathrm{P} \\
\mathrm{R} \\
\mathrm{O} \\
\mathrm{B}\end{array}$} & $\begin{array}{l}0.50 \\
0.50 \\
0.50\end{array}$ & & $\begin{array}{l}0.50 \\
0.50 \\
0.50\end{array}$ & \\
\hline & & $\begin{array}{l}1.00 \\
0.50 \\
0.50 \\
\end{array}$ & $\begin{array}{l}1.00 \\
0.50 \\
0.50 \\
\end{array}$ & \\
\hline & $\begin{array}{l}0.50 \\
0.50 \\
0.00 \\
\end{array}$ & & & $\begin{array}{l}0.50 \\
0.50 \\
0.00\end{array}$ \\
\hline & & $\begin{array}{l}0.50 \\
0.50 \\
0.50 \\
\end{array}$ & & $\begin{array}{l}0.50 \\
0.50 \\
0.50\end{array}$ \\
\hline Score per Problem & 2.50 & 3.25 & 3.25 & 2.50 \\
\hline Number of Students & 6.00 & 6.00 & 6.00 & 6.00 \\
\hline Score per Student & 0.42 & 0.54 & 0.54 & 0.42 \\
\hline \multicolumn{5}{|c|}{ Rubric } \\
\hline $\begin{array}{l}\text { Low Ability } \\
\text { Medium Ability } \\
\text { High Ability }\end{array}$ & $\begin{array}{l}0.00 \\
0.50 \\
1.00\end{array}$ & $\begin{array}{l}0.00 \\
0.50 \\
1.00\end{array}$ & $\begin{array}{l}0.00 \\
0.50 \\
1.00\end{array}$ & $\begin{array}{l}0.00 \\
0.50 \\
1.00\end{array}$ \\
\hline
\end{tabular}

\begin{tabular}{|c|c|c|c|}
\hline & Tonifer Group & Actual Score & 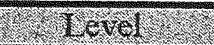 \\
\hline Summary & $\begin{array}{l}\text { Male } \\
\text { Female } \\
\text { Fully } \\
\text { Partially }\end{array}$ & $\begin{array}{l}0.42 \\
0.54 \\
0.54 \\
0.42\end{array}$ & $\begin{array}{l}\text { Medium } \\
\text { Mcdrun } \\
\text { Mediun } \\
\text { Medrun }\end{array}$ \\
\hline
\end{tabular}

\begin{tabular}{|l|l|}
\hline Cometusion & $\begin{array}{l}\text { Males and students in group partially constructing models } \\
\text { improved sketching abilities from low-medium to medium } \\
\text { in three weeks. }\end{array}$ \\
\hline
\end{tabular}




\section{APPENDIX G}

\section{PSVT Results for Pilot Studies}

Two pilot studies were conducted prior to the present study. The first pilot was conducted in the Partnership for Academic Communities (PAC) program at Florida International University in Miami. This program focuses on an intensive math, science, and technology curriculum for at-risk, public-school students from seventh to twelfth grades. In this program, students are selected from a south Miami-Dade county community to attend classes every morning at the university during the academic year. The students return to their home schools after taking approximately one hour of each of the three subjects. Every student who participated in the study had at least two years of experience using GSP.

The Purdue Spatial Visualization Test (PSVT) was administered before and after an eight-week treatment to seventeen tenth-grade students in a geometry class. The treatment consisted in constructing models of polyhedra in GSP and with manipulatives in a constructivist class setting. Each construction was coupled to mathematical questions about the properties and relationships of solids. In groups of two or three, students collaborated to solve the problems posed. The results of a one-way ANCOVA showed no significant differences between males and females, $F(1,14)=0.18, p=0.676$. Results from a paired-samples $t$-test, $t(16)=3.98, p=0.001$, showed a significant difference between pretest and posttest scores. An improvement in the mean PSVT scores from 12.53 points in the pretest to 17.41 in the posttest was observed. The pilot study at the PAC program helped the researcher identify which tasks were more engaging and which cognitive processes the students were most likely to use when solving the treatment tasks. 
From this information, the qualitative data collection forms and the six lessons of the actual study were designed.

The second pilot study included ninety-three tenth-grade students with no prior experience with GSP and registered in three regular geometry classes in the same public school where the dissertation study was conducted. This second pilot study had the purpose of structuring the data analysis techniques by using data collection forms suitable for the methods of analysis described in chapter three of this dissertation. The classes were assigned to a control group and two experimental groups receiving the same treatment as for the dissertation, but lasting seven weeks. The treatment used tasks that encouraged students to connect visual and analytic reasoning. Significant differences were not found for group or gender.

Table G1

PSVT Scores from Pilot Study at PAC Program

\begin{tabular}{|c|c|r|r|}
\hline Student No. & Gender & PreTot & Post Tot \\
\hline 1 & 1 & 15 & 26 \\
2 & 1 & 14 & 7 \\
3 & 1 & 6 & 8 \\
4 & 1 & 14 & 21 \\
5 & 1 & 11 & 17 \\
6 & 1 & 15 & 22 \\
7 & 1 & 6 & 13 \\
8 & 1 & 16 & 21 \\
9 & 1 & 11 & 22 \\
10 & 0 & 18 & 22 \\
11 & 0 & 12 & 18 \\
12 & 0 & 16 & 15 \\
13 & 0 & 7 & 15 \\
14 & 0 & 16 & 25 \\
15 & 0 & 11 & 19 \\
16 & 0 & 14 & 19 \\
17 & 0 & 11 & 6 \\
\hline
\end{tabular}

Note. Female $=0$ and Male $=1$. 
Pilot Study at a Public High School

Table G2

Control Group

\begin{tabular}{|r|r|r|r|r|r|r|r|}
\hline \multirow{2}{*}{ No. Gender } & \multicolumn{3}{|c|}{ Pretest } & \multicolumn{3}{|c|}{ Posttest } \\
\cline { 2 - 7 } & DEV & ROT & VIE & DEV & ROT & VIE \\
2 & 1 & 4 & 5 & 4 & 7 & 7 & 7 \\
3 & 1 & 2 & 4 & 2 & 5 & 8 & 4 \\
4 & 1 & 2 & 5 & 4 & 2 & 6 & 2 \\
5 & 0 & 5 & 6 & 6 & 5 & 8 & 10 \\
6 & 0 & 5 & 2 & 1 & 4 & 1 & 3 \\
7 & 1 & 3 & 8 & 9 & 11 & 11 & 11 \\
8 & 0 & 5 & 6 & 10 & 7 & 6 & 8 \\
9 & 1 & 3 & 6 & 10 & 6 & 12 \\
10 & 1 & 4 & 8 & 3 & 6 & 7 & 5 \\
11 & 1 & 4 & 4 & 3 & 4 & 8 & 5 \\
12 & 0 & 6 & 1 & 3 & 7 & 6 & 8 \\
13 & 1 & 0 & 4 & 3 & 3 & 4 & 4 \\
14 & 1 & 7 & 8 & 7 & 4 & 3 & 1 \\
15 & 0 & 3 & 4 & 2 & 3 & 3 & 10 \\
16 & 1 & 2 & 1 & 1 & 3 & 7 & 7 \\
17 & 0 & 2 & 2 & 2 & 2 & 5 & 3 \\
18 & 1 & 4 & 8 & 1 & 5 & 5 & 9 \\
19 & 1 & 5 & 1 & 4 & 3 & 5 & 4 \\
20 & 0 & 3 & 3 & 1 & A B S N T \\
21 & 0 & 2 & 1 & 1 & 4 & 6 & 3 \\
22 & 0 & 1 & 4 & 6 & 5 & 4 & 5 \\
23 & 1 & 4 & 8 & 8 & 4 & 6 & 10 \\
24 & 0 & 5 & 7 & 1 & 1 & 4 & 4 \\
25 & 0 & 5 & 4 & 2 & 2 & 3 & 4 \\
26 & 1 & 8 & 2 & 6 & 10 & 6 & 2 \\
27 & 0 & 7 & 0 & 6 & 4 & 4 & 3 \\
28 & 1 & 3 & 1 & 2 & 2 & 2 & 0 \\
29 & 0 & 4 & 4 & 3 & 1 & 3 & 2 \\
30 & 1 & 4 & 1 & 1 & 2 & 1 & 2 \\
31 & 1 & 6 & 8 & 2 & 9 & 8 & 6 \\
\hline
\end{tabular}

Note. Female $=0$ and Male $=1$. 
Table G3

Fully Constructing Models with GSP (Experimental Group I)

\begin{tabular}{|c|c|c|c|c|c|c|c|}
\hline \multirow[b]{2}{*}{ No. } & \multirow[b]{2}{*}{ Gender } & \multicolumn{3}{|c|}{ Pretest } & \multicolumn{3}{|c|}{ Posttest } \\
\hline & & DEV & ROT & $\overline{\text { VIE }}$ & $\overline{\mathrm{DEV}}$ & ROT & $\overline{\mathrm{VIE}}$ \\
\hline 1 & 0 & $\overline{5}$ & 1 & $\overline{7}$ & 1 & 4 & 10 \\
\hline 2 & 1 & 8 & 5 & 2 & 8 & 5 & 1 \\
\hline 3 & 0 & 7 & 5 & 9 & 9 & 10 & 6 \\
\hline 4 & 0 & 3 & 2 & 3 & 3 & 3 & 1 \\
\hline 5 & 1 & 2 & 3 & 3 & 3 & 6 & 2 \\
\hline 6 & 1 & 4 & 5 & 7 & 4 & 5 & 5 \\
\hline 7 & 1 & 3 & 4 & 2 & 6 & 4 & 4 \\
\hline 8 & 1 & 2 & 1 & 1 & 8 & 2 & 3 \\
\hline 9 & 1 & 8 & 8 & 7 & 9 & 9 & 6 \\
\hline 10 & 0 & 0 & 8 & 10 & 4 & 2 & 7 \\
\hline 11 & 0 & 2 & 5 & 1 & 3 & 0 & 0 \\
\hline 12 & 1 & 3 & 5 & 4 & 4 & 3 & 7 \\
\hline 13 & 0 & 3 & 4 & 3 & 1 & 3 & 0 \\
\hline 14 & 0 & 2 & 2 & 1 & 5 & 3 & 2 \\
\hline 15 & 1 & 2 & 2 & 2 & 2 & 5 & 1 \\
\hline 16 & 0 & 4 & 3 & 5 & 5 & 5 & 6 \\
\hline 17 & 1 & 9 & 9 & 10 & 12 & 10 & 9 \\
\hline 18 & 1 & 3 & 2 & 3 & 4 & 3 & 4 \\
\hline 19 & 0 & 7 & 7 & 9 & 8 & 5 & 10 \\
\hline 20 & 1 & 4 & 1 & 1 & 2 & 1 & 3 \\
\hline 21 & 0 & 4 & 1 & 1 & 5 & 3 & 2 \\
\hline 22 & 0 & 1 & 2 & 3 & 5 & 4 & 5 \\
\hline 23 & 0 & 6 & 1 & 6 & 7 & 8 & 9 \\
\hline 24 & 1 & 4 & 4 & 6 & 9 & 4 & 6 \\
\hline 25 & 0 & 7 & 3 & 6 & 8 & 8 & 9 \\
\hline 26 & 0 & 6 & 3 & 11 & 7 & 3 & 10 \\
\hline 27 & 1 & 6 & 6 & 2 & 4 & 5 & 4 \\
\hline 28 & 0 & 2 & 2 & 4 & 2 & 2 & 3 \\
\hline
\end{tabular}

Note. Female $=0$ and Male $=1$. 
Table G4

Partially Constructing Models with GSP (Experimental Group 2)

\begin{tabular}{|c|c|c|c|c|c|c|c|}
\hline \multirow[b]{2}{*}{ No. } & \multirow[b]{2}{*}{ Gender } & \multicolumn{3}{|c|}{ Pretest } & \multicolumn{3}{|c|}{ Posttest } \\
\hline & & $\mathrm{DEV}$ & ROT & VIE & $\mathrm{DEV}$ & ROT & VIE \\
\hline 1 & 1 & 3 & 3 & 6 & 7 & 4 & 9 \\
\hline 2 & 0 & 1 & 7 & 3 & 1 & 5 & 1 \\
\hline 3 & I & 4 & 4 & 9 & 5 & 9 & 7 \\
\hline 4 & 1 & 5 & 6 & 3 & 5 & 11 & 7 \\
\hline 5 & 1 & 7 & 7 & 4 & 4 & 6 & 5 \\
\hline 6 & 0 & 9 & 6 & 9 & 12 & 9 & 10 \\
\hline 7 & 0 & 3 & 3 & 1 & 1 & 2 & 4 \\
\hline 8 & 1 & 3 & 6 & l & 5 & 6 & 9 \\
\hline 9 & 0 & 8 & 3 & 2 & 9 & 6 & 2 \\
\hline 10 & 1 & 5 & 9 & 4 & 6 & 11 & 6 \\
\hline 11 & I & 2 & 7 & 2 & 6 & 9 & 9 \\
\hline 12 & 1 & 0 & 4 & 0 & 1 & 4 & 1 \\
\hline 13 & 1 & 10 & 3 & 8 & 11 & 8 & 8 \\
\hline 14 & 1 & 3 & 4 & 7 & 3 & 4 & 10 \\
\hline 15 & 0 & 7 & 5 & 6 & 5 & 5 & 7 \\
\hline 16 & 1 & 4 & 5 & 4 & 9 & 7 & 11 \\
\hline 17 & 0 & 3 & 6 & 6 & 8 & 7 & 8 \\
\hline 18 & 1 & 5 & 7 & 5 & 11 & 9 & 6 \\
\hline 19 & 1 & 5 & 1 & 1 & 8 & 6 & 11 \\
\hline 20 & 1 & 4 & 5 & 2 & 1 & 3 & 2 \\
\hline 21 & 1 & 7 & 0 & 3 & 0 & 0 & 3 \\
\hline 22 & 1 & 8 & 7 & 2 & 11 & 9 & 0 \\
\hline 23 & 1 & 2 & 2 & 3 & 5 & 6 & 2 \\
\hline 24 & 1 & 4 & 4 & 2 & 4 & 3 & 2 \\
\hline 25 & 1 & 6 & 9 & 2 & 9 & 7 & 8 \\
\hline 26 & 1 & 5 & 4 & 6 & 3 & 5 & 6 \\
\hline 27 & 1 & 5 & 8 & 7 & 5 & 8 & 7 \\
\hline 28 & 1 & 9 & 10 & 8 & 11 & 9 & 11 \\
\hline 29 & 1 & 3 & 3 & 7 & 5 & 8 & 4 \\
\hline 30 & 1 & 6 & 9 & 3 & 7 & 10 & 4 \\
\hline 31 & 1 & 8 & 5 & 2 & 11 & 8 & 3 \\
\hline 32 & 0 & 7 & 2 & 5 & 2 & 2 & 6 \\
\hline 33 & 1 & 2 & 2 & 1 & 4 & 9 & 4 \\
\hline 34 & 0 & 4 & 1 & 4 & 5 & 4 & 1 \\
\hline 35 & 0 & 6 & 9 & 5 & 8 & 4 & 5 \\
\hline
\end{tabular}

Note. Female $=0$ and Male $=1$. 


\section{APPENDIX H}

\section{Protocol and GSP Problems}

\section{Protocol Problems}

\section{Problem \#1}

Given an octahedron with edge $s=1$, find its height (distance between opposite
vertices).

\section{Problem \#2}

Given $x$, the area of a polygon, and $y$, the area of another (different) polygon, construct a three-dimensional object whose surface area, $A=2(x+2 y)$.

Problem \#3

a. A 100-story building in New York is a rectangular prism with a square base. Express its surface area using two variables. Include both top and bottom squares. b. Find an expression for the surface area of a Polydron prism made of four rectangles and two squares.

\section{Problem $\# 4$}

Given a cube, find the ratio of the area of one of its square faces to the area of the square cross-section of the octahedron which is the dual of the given cube.

\section{GSP Problems}

Problem \#5

A smaller cube is the dual of the dual of a larger cube. What is the ratio of the edge length of the larger cube to the edge length of the smaller cube?

Problem \#6

A solid is constructed by connecting the midpoints of all edges of a regular tetrahedron and by hiding certain features of the tetrahedron. What is the name of the solid thus formed? What is the ratio of the edge length of the tetrahedron to the edge length of the new solid?

Problem \#7

The icosahedron and dodecahedron are duals of each other. What is the number of pentagonal cross-sections of $s=1$ in an icosahedron of $s=1$ ?

Problem $\# 10$

A smaller tetrahedron is the dual of the dual of a larger tetrahedron. What is the ratio of the volume of the larger tetrahedron to the volume of the smaller tetrahedron? 


\section{APPENDIX I}

\section{GSP Data}

GSP Problem \#5

\begin{tabular}{|c|c|c|c|}
\hline Type & $\begin{array}{l}\text { Number of } \\
\text { Types in } \\
\text { Group El* }\end{array}$ & $\begin{array}{l}\text { Number of } \\
\text { Types in } \\
\text { Group E2* }\end{array}$ & Description of Solution \\
\hline A & $\begin{array}{c}4 \\
3 \mathrm{M} 1 \mathrm{~F}(*)\end{array}$ & $\begin{array}{c}4 \\
2 \mathrm{M} 2 \mathrm{~F}\end{array}$ & $\begin{array}{l}\text { Incorrect solution. } \\
\text { Locates incorrectly centroid on faces } \\
\text { of octahedron. }\end{array}$ \\
\hline B & $\begin{array}{c}1 \\
0 \mathrm{M} I \mathrm{~F}(* *) \\
\end{array}$ & $\begin{array}{c}0 \\
\text { OMOF }\end{array}$ & $\begin{array}{l}\text { Correct solution by using GSP measure tool. } \\
\text { Uses size instead of length when reporting } \\
\text { ratio. }\end{array}$ \\
\hline C & $\begin{array}{c}1 \\
0 \mathrm{M} H \mathrm{~F} \\
\end{array}$ & $\begin{array}{c}1 \\
\text { OMlF }\end{array}$ & $\begin{array}{l}\text { Incorrect solution. } \\
\text { Locates correctly centroid on faces of } \\
\text { octahedron. Does not provide answer for ratio. }\end{array}$ \\
\hline $\mathrm{K}$ & $\begin{array}{c}0 \\
O M O F\end{array}$ & $\begin{array}{c}1 \\
\text { IMOF }\end{array}$ & Leaves blank. \\
\hline & 6 & 6 & Students Missing: 0 \\
\hline
\end{tabular}

Note $\left({ }^{*}\right)$. E1 represents the group fully constructing models with GSP and

E2 the group partially constructing models with GSP.

GSP Problem \#6

\begin{tabular}{|c|c|c|c|}
\hline Type & $\begin{array}{l}\text { Number of } \\
\text { Types in } \\
\text { Group E1 }\end{array}$ & $\begin{array}{l}\text { Number of } \\
\text { Types in } \\
\text { Group E2 }\end{array}$ & Description of Solution \\
\hline D & $\begin{array}{c}5 \\
3 \mathrm{M} 2 \mathrm{~F} \\
\end{array}$ & $\stackrel{1}{O M I F}$ & $\begin{array}{l}\text { Incorrect solution. } \\
\text { Identifies octahedron, but calls it a dual. } \\
\text { Does not provide answer for ratio. }\end{array}$ \\
\hline $\mathrm{E}$ & $\begin{array}{c}1 \\
0 \mathrm{M} 1 \mathrm{~F}\end{array}$ & $\begin{array}{c}0 \\
0 \mathrm{M} 0 \mathrm{~F}\end{array}$ & $\begin{array}{l}\text { Incorrect solution. } \\
\text { Identifies octahedron without calling it dual. } \\
\text { Does not provide answer for ratio. }\end{array}$ \\
\hline $\mathrm{F}$ & $\begin{array}{c}0 \\
\text { OMOF }\end{array}$ & $\stackrel{3}{2 \mathrm{M} I F}$ & $\begin{array}{l}\text { Correct solution. } \\
\text { Answers by estimating visually. }\end{array}$ \\
\hline G & $\begin{array}{c}0 \\
\mathrm{OMOF}\end{array}$ & $\stackrel{1}{0 M 1 F}$ & $\begin{array}{l}\text { Incorrect solution. } \\
\text { Uses GSP measure tool. }\end{array}$ \\
\hline K & $\begin{array}{c}0 \\
\mathrm{OMOF}\end{array}$ & $\stackrel{1}{\mathrm{MMOF}}$ & Leaves blank. \\
\hline & 6 & 6 & Students Missing: 0 \\
\hline
\end{tabular}

Note $\left(^{*}\right) .3$ males and 1 female working in group.

Note $\left({ }^{* *}\right) .1$ female working individually. 
GSP Problem \#7

\begin{tabular}{|c|c|c|l|}
\hline Type & $\begin{array}{c}\text { Number of } \\
\text { Types in } \\
\text { Group E1 }\end{array}$ & $\begin{array}{c}\text { Number of } \\
\text { Types in } \\
\text { Group E2 }\end{array}$ & \\
\hline $\mathrm{H}$ & $\begin{array}{c}0 \\
0 \mathrm{M} 0 \mathrm{~F}\end{array}$ & $\begin{array}{c}1 \\
0 \mathrm{MLF}\end{array}$ & $\begin{array}{l}\text { Correct solution. } \\
\text { Shades one pentagonal cross-section } \\
\text { of the icosahedron and visualizes others. }\end{array}$ \\
\hline $\mathrm{K}$ & $\begin{array}{c}5 \\
2 \mathrm{M} 3 \mathrm{~F}\end{array}$ & $\begin{array}{c}\text { Leaves blank } \\
\mathrm{M}\end{array}$ & \\
\hline & 5 & 4 & Students Missing: 3 \\
\hline
\end{tabular}

GSP Problem \#10

\begin{tabular}{|c|c|c|c|}
\hline Type & $\begin{array}{l}\text { Number of } \\
\text { Types in } \\
\text { Group E1 }\end{array}$ & $\begin{array}{l}\text { Number of } \\
\text { Types in } \\
\text { Group E2 }\end{array}$ & Description of Solution \\
\hline I & $\begin{array}{c}6 \\
3 \mathrm{M} 3 \mathrm{~F} \\
\end{array}$ & $\begin{array}{c}4 \\
2 \mathrm{M} 2 \mathrm{~F}\end{array}$ & $\begin{array}{l}\text { Incorrect solution. } \\
\text { Answers } 1: 9 \text { ignoring dual of dual } \\
\text { (uses } 3 \times 3 \text { instead of } 3 \times 3 \times 3 \text { ). }\end{array}$ \\
\hline$J$ & $\begin{array}{c}0 \\
\text { OMOF }\end{array}$ & $\begin{array}{c}1 \\
0 \mathrm{M} 1 \mathrm{~F}\end{array}$ & $\begin{array}{l}\text { Incorrectly uses result of another problem. } \\
\text { (see Appendix } J \text {, field note } \# 7 \text { a where } 1 \mathrm{~T}=8 \mathrm{t} \text { ). } \\
\text { Answers } 1: 8: 64 \text { considering dual of dual. }\end{array}$ \\
\hline K & $\begin{array}{c}0 \\
\text { OMOF }\end{array}$ & $\stackrel{1}{1 \mathrm{M} 0 \mathrm{~F}}$ & Leaves blank. \\
\hline & 6 & 6 & Students Missing: 0 \\
\hline
\end{tabular}




\section{APPENDIX J}

Field Notes

Day \#1

Label: $\quad$ Connecting Points

Period: $\quad 4^{\text {th }}$ (Group partially constructing models in GSP)

Context: L\#0 (GSP Tutorial)

Problem: Construct a square and an equilateral triangle.

Observations: A student attempts to construct a square and an equilateral triangle by first constructing points approximately every 10 degrees on the circumference of a circle, and then connecting some of the points. This shows the student's notion of constructing a figure is not yet developed.

AV comment: Uses visual reasoning (V) as an alternative to analytic reasoning (A).

\begin{tabular}{|l|l|l|l|l|l|l|l|l|l|}
\hline Day & C\&S & TRA & DIL & ROT & REF & SLI & FOL & CON & INT \\
\hline D1 & & & & $\checkmark$ & & & & $\checkmark$ & \\
\hline
\end{tabular}

Day \#2

Label: $\quad$ Unit as a Variable

Period: $\quad 2^{\text {nd }}$ (Group fully constructing models in GSP)

Context: $\quad$ L\#1 (Polydron Manipulatives)

Problem: Identify the unit for expressing the surface area (SA) and volume (VOL) of a solid. Write expressions for SA and VOL.

Observations: A student insists in using SA and VOL formulas learned in previous courses. The student insists in using $\mathrm{SA}=\mathrm{w} \times \mathrm{l}$ and $\mathrm{VOL}=\mathrm{w} \times 1 \times \mathrm{h}$. When working on expressing a cube's volume with the use of unit variables, one student asked another what $\mathrm{s}$ in $\mathrm{s}^{3}$ meant. The second student responded, "s is for square."

AV comment: Use of A blocks V.

\begin{tabular}{|l|l|l|l|l|l|l|l|l|l|}
\hline Day & C\&S & TRA & DIL & ROT & REF & SLI & FOL & CON & INT \\
\hline D2 & $\checkmark$ & & & & & $\checkmark$ & & & \\
\hline
\end{tabular}


Day \#3a

Label: $\quad$ Three-Dimensional Cross Growth

Period: $\quad 2^{\text {nd }}$

Context: $\quad$ L\#1 (Color Blocks)

Problem: Discover a pattern for the expressions of SA and VOL as the 3-D cross grows in size. Start with one cube. Continue by attaching another cube to all faces of the first cube. Continue attaching cubes to all faces of the six new cubes. Continue indefinitely.

Observations: A student finds the expressions for SA and VOL only for the first and second terms of the iteration. The student cannot generalize and discover the pattern.

AV comment: Use of $\mathrm{V}$, weak use of $\mathrm{A}$.

\begin{tabular}{|l|l|l|l|l|l|l|l|l|l|}
\hline Day & C\&S & TRA & DIL & ROT & REF & SLI & FOL & CON & INT \\
\hline D3a & $\checkmark$ & & & & $\checkmark$ & $\checkmark$ & & & \\
\hline
\end{tabular}

Day \#3b

Label: $\quad$ Counting Unit Areas of Icosahedron

Period: $\quad 2^{\text {nd }}$ and $4^{\text {th }}$

Context: $\quad$ L\#1 (Polydron Manipulatives)

Problem: Write an expression for the surface area of an icosahedron, using equilateral triangles as the unit of area.

Observations: A student loses track of his unit count (triangles). The student then relies on multiplication to support counting. The student counts five units at a time, and uses ROT and REF to keep track of count.

AV comment: $\mathrm{V}$ supports $\mathrm{A}$.

\begin{tabular}{|l|l|l|l|l|l|l|l|l|l|}
\hline Day & C\&S & TRA & DIL & ROT & REF & SLI & FOL & CON & INT \\
\hline D3b & & & & $\checkmark$ & $\checkmark$ & & & & \\
\hline
\end{tabular}

Day \#4a

Label: $\quad$ Ratio of Volume Q to Volume T

Period: $\quad$ N/A (Not Available)

Context: $\quad$ L\#2 (Polydron Manipulatives)

Problem: $\quad$ Prove $1 \mathrm{Q}=4 \mathrm{~T}$, where $\mathrm{Q}$ is the volume of an octahedron of $\mathrm{s}=1$ and $\mathrm{T}$ is the volume of a tetrahedron of $s=1$.

Observations: Student uses conclusion as part of the premise.

AV comment: N/A

\begin{tabular}{|l|l|l|l|l|l|l|l|l|l|}
\hline Day & C\&S & TRA & DIL & ROT & REF & SLI & FOL & CON & INT \\
\hline D4a & $\checkmark$ & & $\checkmark$ & & & & & & \\
\hline
\end{tabular}


Day \#4b

Label: $\quad$ Reconstruct a Solid without Looking at the Model

Period: $\quad 2^{\text {nd }}$

Context: L\#1 (Polydron Manipulatives)

Problem: Reconstruct the solid labeled "heart" given 16 equilateral triangles.

Observations: A student uses a net to support memory, and constructs the "heart."

AV comment: V supports A.

\begin{tabular}{|l|l|l|l|l|l|l|l|l|l|}
\hline Day & C\&S & TRA & DIL & ROT & REF & SLI & FOL & CON & INT \\
\hline D4b & $\checkmark$ & & & $\checkmark$ & $\checkmark$ & & $\checkmark$ & $\checkmark$ & \\
\hline
\end{tabular}

Day \#4c

Label: $\quad$ Ratio of Volume P to Volume T

Period: N/A

Context: $\quad$ L\#2 (Polydron Manipulatives)

Problem: $\quad$ Prove the relation between $P$ (the volume of a square pyramid of $s=1$ ) and $T$ (the volume of a tetrahedron of $s=1$ ). That is, prove $1 \mathrm{P}=2 \mathrm{~T}$.

Observations: A student thinks the solution of this problem leads to the solution $1 \mathrm{Q}=4 \mathrm{~T}$ investigated in problem $\# 4 \mathrm{a}$ above. The student knows that $2 \mathrm{P}=1 \mathrm{Q}$, and claims that $1 \mathrm{P}=$ $2 \mathrm{~T}$. The student uses transitivity to express: $2 \mathrm{P}=4 \mathrm{~T}=1 \mathrm{Q}$. The claim is justified visually by removing the square base of square pyramid, and then manipulating the four remaining triangles. The four vertices from where the square base was removed still forms a square. The student enlarges the distance between two opposite vertices while the distance between the two other opposite vertices contracts. The student stops this stretchcontract process when the dihedral angle between two triangles reaches the dihedral angle of a tetrahedron.

AV comment: V supports A. The student uses conservation of volume intuitively.

\begin{tabular}{|l|l|l|l|l|l|l|l|l|l|}
\hline Day & C\&S & TRA & DIL & ROT & REF & SLI & FOL & CON & INT \\
\hline D4c & $\checkmark$ & & & & $\checkmark$ & $\checkmark$ & $\checkmark$ & & \\
\hline
\end{tabular}

Day \#5

N/A

Day \#6

N/A 
Day \#7a

$\begin{array}{ll}\text { Label: } & \text { Support Problem for Proof Problem }(1 T=8 t) \\ \text { Period: } & 2^{\text {nd }}\end{array}$

Context: $\quad$ L\#2 (Polydron Manipulatives)

Problem: What solid results after removing the volume $(t)$ of 4 small tetrahedra from the volume $(\mathrm{T})$ of a larger tetrahedron (the ratio of the small tetrahedra edge lengths to larger tetrahedron's edge lengths is $1: 2$ )?

Observations: The student visualizes the solid after performing mental slices.

AV comment: A supports V.

\begin{tabular}{|l|l|l|l|l|l|l|l|l|l|}
\hline Day & C\&S & TRA & DIL & ROT & REF & SLI & FOL & CON & INT \\
\hline D7a & $\checkmark$ & & $\checkmark$ & $\checkmark$ & & $\checkmark$ & & & $\checkmark$ \\
\hline
\end{tabular}

Day \#7b

Label: $\quad$ Counting Unit Volumes in a Cube

Period: $\quad 4^{\text {th }}$

Context: $\quad$ L\#1 (Color Blocks)

Problem: After lining up 5 smaller cubes in each of the three directions $\mathrm{x}, \mathrm{y}$, and $\mathrm{z}$ (one cube is shared by all axes), write an expression for the volume of the resulting larger cube, using $\mathrm{c}$, the volume of one of the smaller cubes, as the unit of volume.

Observations: The student starts counting one by one and expresses the volume as $124 \mathrm{c}$. After the teacher lets the student know there was a mistake in counting, the student relies on multiplication to support the counting of 25 small cubes at a time. The student uses $\mathrm{C} \& \mathrm{~S}$ and $\mathrm{CON}$ while counting.

AV comment: V supports A.

\begin{tabular}{|l|l|l|l|l|l|l|l|l|l|}
\hline Day & C\&S & TRA & DIL & ROT & REF & SLI & FOL & CON & INT \\
\hline D7b & $\checkmark$ & & & & & & & $\checkmark$ & \\
\hline
\end{tabular}


Day \#8a

Label: $\quad$ Dual of the Dual of a Cube

Period: 2nd

Context: $\quad$ L\#4

Problem: Given the definition of dual, find the dual of the cube and the dual of the cube's dual.

Observations: The student is able to identify both by visualizing them.

AV comment: A supports V.

\begin{tabular}{|l|l|l|l|l|l|l|l|l|l|}
\hline Day & C\&S & TRA & DIL & ROT & REF & SLI & FOL & CON & INT \\
\hline D8a & $\checkmark$ & & & & & & & $\checkmark$ & \\
\hline
\end{tabular}

Day \#8b

Label: $\quad$ Ratio of the Length of the Diagonal to the length of a Cube's Edge $(s=1)$ Period: $\quad 2^{\text {nd }}$

Context: $\quad$ L4 (GSP)

Problem: $\quad$ Find the length of the diagonal of a cube whose edges measure 1 unit.

Observations: The student drags the control button until the display for the cube's edge lengths read 1 unit. Then, by using the measure tool, the student answers, "d equals 1.403 units."

AV comment: V only.

\begin{tabular}{|l|l|l|l|l|l|l|l|l|l|}
\hline Day & C\&S & TRA & DIL & ROT & REF & SLI & FOL & CON & INT \\
\hline D8b & & & $\checkmark$ & $\checkmark$ & & & & $\checkmark$ & \\
\hline
\end{tabular}


Day \#8c

Label: $\quad$ Dual of a Cube

Period: $\quad 4^{\text {th }}$

Context: $\quad$ L\#4 (GSP)

Problem: Given the definition of dual, sketch the dual of the cube.

Observations: The student draws a center point for each of the six faces of the cube on a whiteboard. The student connects four points, forming a square. The student cannot proceed and says: "I can't do it here, but I know it is a diamond and I am going to construct it with GSP in my computer."

AV comment: A does not support V.

\begin{tabular}{|l|l|l|l|l|l|l|l|l|l|}
\hline Day & C\&S & TRA & DIL & ROT & REF & SLI & FOL & CON & INT \\
\hline D8c & & & & $\checkmark$ & & & & $\checkmark$ & $\checkmark$ \\
\hline
\end{tabular}

Day \#8d

Label: $\quad$ Ratio of Lenth of the Diagonal to Length of Edges of a Cube $(e=5)$

Period: $\quad 4^{\text {th }}$

Context: $\quad$ L\#4

Problem: Find the length of the diagonal of a cube whose edges measure 5.

Observations: The student responds, " 5 x 1.73 since $d=1.73$ for the case of $e=1$."

AV comment: A only.

\begin{tabular}{|l|l|l|l|l|l|l|l|l|l|}
\hline Day & C\&S & TRA & DIL & ROT & REF & SLI & FOL & CON & INT \\
\hline D8d & & & $\checkmark$ & & & & & & \\
\hline
\end{tabular}


Day \#8e

Label: $\quad$ Diagonal of a Cube

Period: $\quad 2^{\text {nd }}$ and

$4^{\text {th }}$

Context: $\quad$ L\#4 (GSP)

Problem: Given a cube constructed in GSP, construct its diagonal.

Observations: The student constructs a diagonal on a square face of the cube. The student repeats this twice again despite being reminded by the teacher that the problem is about a cube. The student rotates the orientation of the cube. On the fourth attempt she correctly constructs the cube's diagonal.

AV comment: $\mathrm{V}$ only.

\begin{tabular}{|l|l|l|l|l|l|l|l|l|l|}
\hline Day & C\&S & TRA & DIL & ROT & REF & SLI & FOL & CON & INT \\
\hline D8e & & & & $\checkmark$ & & & & $\checkmark$ & \\
\hline
\end{tabular}

Day \#9

Label: $\quad$ Inscribed Squares

Period: $\quad 2^{\text {nd }}$

Context: $\quad$ L\#4 (GSP)

Problem: The midpoints of the sides of a square are connected to form a smaller square. The procedure is repeated on the smaller square thus constructed to obtain a third square. What is the ratio of the lengths of the first to third squares?

Observations: Student responds: "Two of those make one of those." Student cannot justify the response.

AV comment: $\mathrm{V}$ only.

\begin{tabular}{|l|l|l|l|l|l|l|l|l|l|}
\hline Day & C\&S & TRA & DIL & ROT & REF & SLI & FOL & CON & INT \\
\hline D9 & & & $\checkmark$ & & & & & & \\
\hline
\end{tabular}


Day \#10a

Label: $\quad$ Volume of Square-Base Rectangular Prism

Period: $\quad 4^{\text {th }}$

Context: $\quad$ L\#3 (Polydron Manipulatives)

Problem: $\quad$ Find the volume of the square-base rectangular prism if the sides of the square are $s=1$. (Hint: Slicing a cube through its diagonal reveals a rectangular cross-section. Find the dimensions of such cross-section.)

Observations: Student tries but cannot solve. After the teacher and student solve the problem together, the student says: "Wow! The rectangles' lengths measure $\sqrt{ } 2$."

AV comment:

\begin{tabular}{|l|l|l|l|l|l|l|l|l|l|}
\hline Day & C\&S & TRA & DIL & ROT & REF & SLI & FOL & CON & INT \\
\hline D10a & $\checkmark$ & & & & & & $\checkmark$ & $\checkmark$ & \\
\hline
\end{tabular}

Day \#10b

Label: $\quad$ Simplifying Radicals

Period: $\quad 2^{\text {nd }}$

Context: $\quad$ L\#3 (Polydron Manipulatives)

Problem: $\quad$ Find the length of the hypotenuse of an isosceles right triangle whose legs measure $s=\sqrt{6}$ (Refer to the penta-hut in problem $3 \mathrm{~b}$ in Appendix B).

Observations: After seeing the teacher simplify the answer $\sqrt{12}$ to $\sqrt{2} \times 2 \times 3$, and then to $2 \sqrt{3}$, a student exclaims, "No one has taught us this before."

AV comment:

\begin{tabular}{|l|l|l|l|l|l|l|l|l|l|}
\hline Day & C\&S & TRA & DIL & ROT & REF & SLI & FOL & CON & INT \\
\hline D10b & & & & & & & $\checkmark$ & & \\
\hline
\end{tabular}


Label: $\quad$ Locating Center of Square Faces of a Cube

Period: $\quad 2^{\text {nd }}$ and $4^{\text {th }}$

Context: L\#4 (GSP)

Problem: Locate the center of a square.

Observations: The student points at center. The teacher constructs the midpoints of the sides of a square to assist the student, and labels them 1,2,3,4 clockwise. The student connects 1-2-3-4 instead of 1-3 and 2-4 to find the intersection between segments 1-3 and 2-4. The teacher connects 1-3. The student then connects $2-4$ and identifies the center of the square.

\section{AV comment:}

\begin{tabular}{|l|l|l|l|l|l|l|l|l|l|}
\hline Day & C\&S & TRA & DIL & ROT & REF & SLI & FOL & CON & INT \\
\hline D11 & & & & & & & & $\checkmark$ & $\checkmark$ \\
\hline
\end{tabular}

Day \#12a

Label: $\quad$ GSP Problem \#8

Period: $\quad 2^{\text {nd }}$

Context: $\quad$ L\#5 (GSP)

Problem: Find the length of segment $\mathrm{AB}$ in the $2 \times 2 \times 2$ grid.

Observations: Student has heard the answer is $\sqrt{3}$ and devices an illogical justification by saying: " $1 / 2+1 / 2+1+1$ is 3 ; then find its square root."

AV comment:

\begin{tabular}{|l|l|l|l|l|l|l|l|l|l|}
\hline Day & C\&S & TRA & DIL & ROT & REF & SLI & FOL & CON & INT \\
\hline D12a & & $\checkmark$ & & & & & & & \\
\hline
\end{tabular}


Day \#12b

Label: $\quad$ Dual of the Dodecahedron

Period: $\quad 2^{\text {nd }}$

Context: L\#4 (Polydron Manipulatives)

Problem: Visualize the dual of the dodecahedron.

Observations: Student responds, "It can't be the octahedron because we need five triangles meeting at a point and the octahedron has four only. Can't be the cube or the tetrahedron, so it is the icosahedron. Oh, yeah, I can see it!"

AV comment:

\begin{tabular}{|l|l|l|l|l|l|l|l|l|l|}
\hline Day & C\&S & TRA & DIL & ROT & REF & SLI & FOL & CON & INT \\
\hline D12b & $\checkmark$ & & & $\checkmark$ & & & & $\checkmark$ & \\
\hline
\end{tabular}

Day \#12c

Label: $\quad$ Dual of the Cube

Period: $\quad 4^{\text {th }}$

Context: $\quad$ L\#4 (Polydron Manipulatives)

Problem: Visualize the dual of the cube.

Observations: Student says, "I don't know; I can't." After another student describes the way she supports her visualization, the first student says, "Oh, I got it now." The teacher asks the first student what the dual of the dodecahedron is and the student immediately knows the answer. Now the teacher asks for the dual of the icosahedron and again the student knows the answer immediately."

AV comment:

\begin{tabular}{|l|l|l|l|l|l|l|l|l|l|}
\hline Day & C\&S & TRA & DIL & ROT & REF & SLI & FOL & CON & INT \\
\hline D12c & $\checkmark$ & & & & $\checkmark$ & & & $\checkmark$ & \\
\hline
\end{tabular}


Label: $\quad$ Constructing a Platonic Solid

Period: $\quad 4^{\text {th }}$

Context: $\quad$ L\#4 (Polydron Manipulatives)

Problem: Given enough equilateral pieces, construct a platonic solid.

Observations: Student observes another student has constructed a tetrahedron using 16 pieces (a two-factor dilation). The first student lets the other student know that using only four pieces is sufficient.

AV comment:

\begin{tabular}{|l|l|l|l|l|l|l|l|l|l|}
\hline Day & C\&S & TRA & DIL & ROT & REF & SLI & FOL & CON & INT \\
\hline D12d & $\checkmark$ & & $\checkmark$ & & & & $\checkmark$ & $\checkmark$ & \\
\hline
\end{tabular}

Day \#13a

Label: $\quad$ GSP Problem \#6

Period: $\quad 4^{\text {th }}$

Context: LH2 (GSP)

Problem: After dilating by a factor of two the edges of a tetrahedron of edges $e=1 / 2$, slice tetrahedra of edges $e=1 / 2$ from each of the four vertices. What is the resulting solid? Find s, the length of the segment connecting two midpoints of two different edges of the enlarged tetrahedron.

Observations: "The small triangle that is $1 / 2$ by $1 / 2$ by $1 / 2$ grows into the larger triangle that is 1 by 1 by 1 . That's how I know that $s=1 / 2 . "$

AV comment:

\begin{tabular}{|l|l|l|l|l|l|l|l|l|l|}
\hline Day & C\&S & TRA & DIL & ROT & REF & SLI & FOL & CON & INT \\
\hline D13a & & & $\checkmark$ & & & & & & \\
\hline
\end{tabular}


Day $\# 13 b$

$\begin{array}{ll}\text { Label: } & \text { GSP Problem \#9 } \\ \text { Period: } & 2^{\text {nd }}\end{array}$

Context: L\#5 (GSP)

Problem: Find the length of the three sides of the triangle and name the type of triangle.

Observations: Student solves for the length of one side by using the Pythagorean theorem as follows: "Two squared plus two squared is eight; so take the square-root and you get the square root of eight. Use the Pythagorean theorem again by adding 1 squared plus eight, which is nine and then square-root it to get the square root of nine." Teacher asks:

"What is the square root of nine?" Student responds, "Oh, three!"

AV comment:

\begin{tabular}{|l|l|l|l|l|l|l|l|l|l|}
\hline Day & C\&S & TRA & DIL & ROT & REF & SLI & FOL & CON & INT \\
\hline D13b & & & & & & & & $\checkmark$ & \\
\hline
\end{tabular}

Day \#13c

Label: $\quad$ GSP Problem \#9

Period: $\quad 2^{\text {nd }}$

Context: $\quad$ L\#5 (GSP)

Problem: Construct a $3 \times 3 \times 2$ grid from the GSP file of a cube.

Observations: Student finds the midpoint first. Then finds the midpoints of the two segments. Then the student construct a grid of 4 by 4 on each face of the cube. Finally, the student hides several rows to get a 3 by 3 by 3 grid. The student says, "If you hide one more row, then you get the 3 by 3 by 2 grid."

AV comment:

\begin{tabular}{|l|l|l|l|l|l|l|l|l|l|}
\hline Day & C\&S & TRA & DIL & ROT & REF & SLI & FOL & CON & INT \\
\hline D13c & $\checkmark$ & $\checkmark$ & $\checkmark$ & $\checkmark$ & & $\checkmark$ & & $\checkmark$ & $\checkmark$ \\
\hline
\end{tabular}


Label: $\quad$ GSP Problem \#9

Period: $\quad 2^{\text {nd }}$

Context: L\#5 (GSP)

Problem: Construct a $3 \times 3 \times 2$ grid from the GSP file of a cube.

Observations: The student translates the cube to construct another cube next to it. The student extends the diagonal of the top face of the given cube with a ray. The student constructs two parallel lines containing the right sides of the top squares of each of the two cubes. By the intersection of one of the parallel lines with the extension of the diagonal of the top square on the given cube, the student locates a point. The student says, "If you repeat this procedure, then you get a cube going into the screen."

AV comment:

\begin{tabular}{|l|l|l|l|l|l|l|l|l|l|}
\hline Day & C\&S & TRA & DIL & ROT & REF & SLI & FOL & CON & INT \\
\hline D13d & $\checkmark$ & $\checkmark$ & & & & & & $\checkmark$ & $\checkmark$ \\
\hline
\end{tabular}

Day\#13e

Label: $\quad$ GSP Problem \#6

Period: $\quad 4^{\text {th }}$

Context: L\#2 (GSP)

Problem: After dilating by a factor of two the edges of a tetrahedron of edges $e=1 / 2$, slice tetrahedra of edges $e=1 / 2$ from each of the four vertices. What is the resulting solid? Find $\mathrm{s}$, the length of the segment connecting two midpoints of two different edges of the enlarged tetrahedron.

Observation: Student uses GSP's tool to measure lengths, and finds one edge to be 1.6 and the other 0.8. The student says, "So, if the large side is one, then $\mathrm{s}=$ 0.5."

AV comment:

\begin{tabular}{|l|l|l|l|l|l|l|l|l|l|}
\hline Day & C\&S & TRA & DIL & ROT & REF & SLI & FOL & CON & INT \\
\hline D13e & & & $\checkmark$ & & & & & & \\
\hline
\end{tabular}

Day \#14

N/A 
Day \#15

Label: $\quad$ Solving in Terms of Variables

Period: $\quad 2^{\text {nd }}$

Context: Protocol Problem \#4 (Written)

Problem: Given a cube, find the ratio between the area of one of its square faces and the square cross-section of the octahedron which is the dual of the given cube.

Observations: "There are no numbers in this problem. It can't be done."

AV comment: No processes checked.

\begin{tabular}{|l|l|l|l|l|l|l|l|l|l|}
\hline Day & C\&S & TRA & DIL & ROT & REF & SLI & FOL & CON & INT \\
\hline D15 & & & & & & & & & \\
\hline
\end{tabular}

Day \#16

N/A 
Table J1

Summary of Usage of Whole-Part Processes in Class Problems

\begin{tabular}{|c|c|c|c|c|c|c|c|c|c|}
\hline $\begin{array}{c}\text { Observation } \\
\text { No. }\end{array}$ & $\mathrm{C} \& \mathrm{~S}$ & TRA & DIL & ROT & REF & SLI & FOL & CON & INT \\
\hline 1 & & & & $\checkmark$ & & & & $\checkmark$ & \\
\hline 2 & $\checkmark$ & & & & & $\checkmark$ & & & \\
\hline 3 & $\sqrt{ }$ & & & & $\checkmark$ & $\checkmark$ & & & \\
\hline 4 & & & & $\checkmark$ & $\checkmark$ & & & & \\
\hline 5 & $\checkmark$ & & $\checkmark$ & & & & & & \\
\hline 6 & $\checkmark$ & & & $\checkmark$ & $\checkmark$ & & $\checkmark$ & $\checkmark$ & \\
\hline 7 & $\checkmark$ & & & & $\checkmark$ & $\checkmark$ & $\checkmark$ & & \\
\hline 8 & $\checkmark$ & & $\checkmark$ & $\checkmark$ & & $\checkmark$ & & & $\checkmark$ \\
\hline 9 & $\checkmark$ & & $\checkmark$ & & & $\checkmark$ & & & \\
\hline 10 & $\checkmark$ & & & & & & & $\checkmark$ & \\
\hline 11 & & & $\checkmark$ & $\checkmark$ & & & & $\checkmark$ & \\
\hline 12 & & & & $\checkmark$ & & & & $\checkmark$ & $\checkmark$ \\
\hline 13 & & & $\checkmark$ & & & & & & \\
\hline 14 & & & & $\checkmark$ & & & & $\checkmark$ & \\
\hline 15 & & & $\checkmark$ & & & & & $\checkmark$ & \\
\hline 16 & $\checkmark$ & & & & & & $\checkmark$ & $\checkmark$ & \\
\hline 17 & & & & & & & $\checkmark$ & & \\
\hline 18 & & & & & & & & $\checkmark$ & $\checkmark$ \\
\hline 19 & & $\checkmark$ & & & & & & & \\
\hline 20 & $\checkmark$ & & & $\checkmark$ & & & & $\checkmark$ & \\
\hline 21 & $\checkmark$ & & & & $\checkmark$ & & & $\checkmark$ & \\
\hline 22 & $\checkmark$ & & $\checkmark$ & & & & $\checkmark$ & $\checkmark$ & \\
\hline 23 & & & $\checkmark$ & & & & & & \\
\hline 24 & & & & & & & & $\checkmark$ & \\
\hline 25 & $\checkmark$ & $\checkmark$ & $\checkmark$ & $\checkmark$ & & $\checkmark$ & & $\checkmark$ & $\checkmark$ \\
\hline 26 & $\checkmark$ & $\checkmark$ & & $\checkmark$ & & & & $\checkmark$ & $\checkmark$ \\
\hline 27 & & & $\checkmark$ & & & & & & \\
\hline 28 & & & $\mathrm{~N}$ & $\mathrm{O}$ & $\mathrm{N}$ & $\mathrm{E}$ & & & \\
\hline
\end{tabular}


VITA

\section{MARIO ERASO}

November 3, 1965

1988

1995

2003
Born, Cali, Colombia

B.S., Civil Engineering

Lehigh University

Bethlehem, Pennsylvania

M.S., Civil Engineering

Lehigh University

Bethlehem, Pennsylvania

M.S., Mathematics Education

Florida International University

Miami, Florida

\section{PUBLICATIONS AND PRESENTATIONS}

Slaughter, E. S., \& Eraso, M. (1997). Simulation of structural steel erection to assess innovations, Transactions on Engineering Management, 44, 196-207.

Eraso, M. (2001, November). Scooting with Technology in the Classroom. Florida Higher Education Consortium $10^{\text {th }}$ Statewide Conference, Saint Petersburg, Florida.

Park, D.Y., O'Brien, G., Eraso, M., and McClintock, E. (2002). A scooter inquiry: An integrated science, mathematics, and technology activity. Science Activities: Classroom Projects and Curriculum Ideas, 39, 27-32.

Eraso, M. (2004, October). The Penta-Hut: A Mathematics Refuge. Florida Council of Teachers of Mathematics $52^{\text {nd }}$ Annual State Conference, Miami, Florida.

Eraso, M. (2005, April). Connecting Algebra and Geometry to Improve Students' Visualization Skills. The $16^{\text {th }}$ International Conference on College Teaching and Learning, Jacksonville, Florida.

Eraso, M. (2005, November). Improving Visualization: The Connections of Visual and Analytic Thinking. School Science and Mathematics Conference, Fort Worth, Texas.

Eraso, M. (2006, November). Meeting with Math Literacy Workers. Teaching for Intelligence: Believe to Achieve Conference, Minneapolis, Minnesota. 